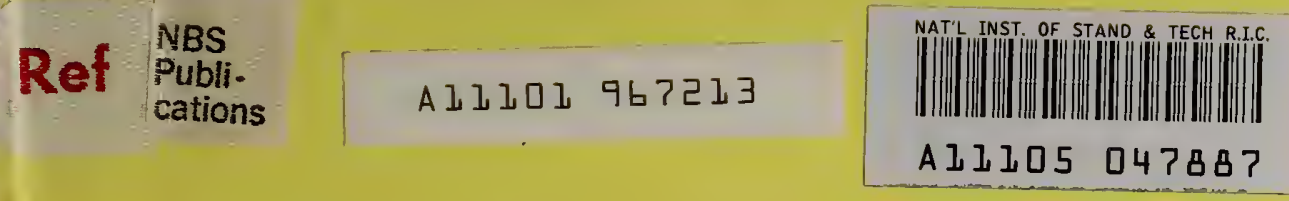

NBSIR 79-1923

\title{
Residential Solar Data Center Grant Reports
}

Patricia M. Christopher

Martin J. Aronoff

Center for Building Technology

National Engineering Laboratory

and

Center for Programming Science and Technology

Institute for Computer Sciences and Technology

National Bureau of Standards

Washington, D.C. 20234

September, 1979

Issued October, 1979

Prepared for:

Department of Housing and Urban Development Office of the Assistant Secretary for Policy Development and Research Division of Energy, Building Technology and Standards

Washington, D.C. 20410 
NBSIR 79-1923

\section{RESIDENTIAL SOLAR DATA CENTER GRANT REPORTS}

Patricia M. Christopher

Martin J. Aronoff

Center for Building Technology

National Engineering Laboratory

and

Center for Programming Science and Technology Institute for Computer Sciences and Technology

National Bureau of Standards

Washington, D.C. 20234

September, 1979

Issued October, 1979

Prepared for:

Department of Housing and Urban Development

Office of the Assistant Secretary for Policy Development and Research Division of Energy, Building Technology and Standards

Washington, D.C. 20410

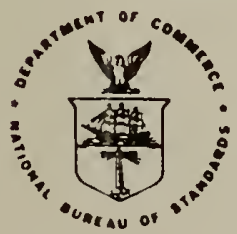

U.S. DEPARTMENT OF COMMERCE, Juanita M. Kreps, Secretary Luther H. Hodges, Jr., Under Secretary

Jordan J. Baruch, Assistant Secretary for Science and Tochnology NATIONAL BUREAU OF STANDARDS, Ernest Ambler, Director 



\section{FOREWORD}

In September 1978, the Residential Solar Data Center (SDC) of the National Bureau of Standards (NBS) issued the publication, "Grant Application Reports" (SDC Report No. 2), a listing of eight sorts of Grant file data collected from Cycles 1,2 and 3 of the Department of Housing and Urban Development's (HUD) Residential Solar Demonstration Program [1]. In March 1979, the SDC issued an updated draft of the same eight types of sorts on data from Cycles $1,2,3,4$ and 4A. These publications received selected distribution to participants (primarily HUD and its contractors) in the Residential Solar Demonstration Program.

This document, Residential Solar Data Center Grant Reports, contains four of the eight computer sorts, significantly reducing its size so that it might be more easily distributed to a larger audience. This publication also has a complete description of the set of data elements included in the computer reports and a description of the relationship between the Grant file and other files maintained by the Solar Data Center. 


\section{ACKNOWLEDGMENTS}

Dr. Blanton C. Duncan, Project Leader and Ms. Mary Lou Hudtloff, Analyst/Programmer of 01d Dominion Systems, Inc. developed the computer programs which were used to produce the Grant file reports.

The authors also wish to thank Ms. Brenda B. Eidson, technical writer of Rehab Group, Inc., for her assistance in editing this document.

The bar graphs of Grant file data in Chapter 5 were produced on the NBS Versatec $1200 \mathrm{~A}$ plotter/printer with programs developed by

Dr. Frank L. McCrackin and, modified by Walter D. Urban, both of NBS. 
RESIDENTIAL SOLAR DATA CENTER

GRANT REPORTS

TABLE OF CONTENTS

Page

FOREWORD . . . . . . . . . . . . . . . . . . iii

ACKNOWLEDGMENTS

iv

1. INTRODUCTION ....................... . . 1

2. GRANT FILE . . . . . . . . . . . . . . . . . . . 1

Definition of the Grant file; how it fits into the solar

Data system; how Grant file data are collected.

3. GRANT FILE DATA ELEMENTS . . . . . . . . . . . . . . 5

Description and values of individual data elements in the

Grant file.

4. GRANT FILE COMPUTER REPORTS . . . . . . . . . . . . . 8

Computer reports that are produced from Grant file data.

Included in this publication are the following:

4.1 "Units and Cost Analysis". . . . . . . . . . . 11

4.2 "Summary of Solar Grants Sorted on Grant Number" • . . 13

4.3 "Summary of Solar Grants Sorted on Manufacturer, with Cost per MBtu" ................. 27

4.4 "Summary of Solar Grants Sorted on Project City and State".................. 44 46

5. GRANT FILE DATA BAR GRAPHS . . . . . . . . . . . 63

6. SUMMARY ........................ 66

7. REFERENCES ..................... . . 67 
RESIDENTIAL SOLAR DATA CENTER

GRANT REPORTS

Patricia M. Christopher

Martin J. Aronoff

\begin{abstract}
The Residential Solar Data Center project staff in the Center for Building Technology, National Bureau of Standards, is responsible for the establishment and operation of a computerized database containing non-instrumented residential data generated by the Solar Heating and Cooling Demonstration Program sponsored by the Department of Energy and the Department of Housing and Urban Development (HUD). This document includes computer reports of data contained in the Grant file, one of six computer files comprising the database. These computer reports contain data recorded on applications submitted to HUD by organizations or individual builders applying for grants to build solar energy systems in new and/or existing homes. To date, approximately 450 grants have been awarded in the first four' awárd cycles.

Key Words: Automatic data processing; computer reports; grant data; residential buildings; solar database; solar energy system; solar heating and cooling.
\end{abstract}




\section{INTRODUCTION}

This report addresses a computer file of data called the Grant file and the computer reports (listings, printouts, graphs, etc.) that are produced from this file of data. Included is a description of the Grant file and its relationship to other files of data in the same database, along with information about how data is collected. Also included are: a detailed description of certain data elements, a description of computer reports produced from the Grant file, and copies of some of these computer reports. Chapter 5 contains copies of a series of bar graphs produced from the same data shown in the computer reports.

\section{GRANT FILE}

The Grant file is one of six computer files which comprise the solar database (see Figure 1) established and maintained by the Residential Solar Data Center (SDC) project staff at the National Bureau of Standards. This computerized database contains data collected for the Residential Solar Heating and Cooling Demonstration Program administered by the Department of Housing and Urban Development (HUD), a major activity in the total Federal Solar Energy Program managed by the Department of Energy [1]*.

Grant file data are initially obtained from completed forms submitted to HUD by organizations or individual builders applying for grants to build solar systems in new homes or to retrofit solar systems into existing homes [2]. Data from these forms are transcribed onto summary sheets (see Figure 2) for entry into the computer. To date, approximately 450 grants have been awarded in four award cycles (the fourth of which had two phases). Cycle 5 applications are being evaluated as of September 1979. A special grant award cycle, the Passive Initiative, was held in December 1978. These data were collected in a slightly different format from the numbered cycles. An effort is currently underway to convert all Grant file data to a common format. This conversion (which should be completed by November 1979) will allow the computer reports to include the Passive Initiative data, as well as all the Cycle 1 through 5 data.

The Grant file contains three types of data: (1) grant level, including the grantee's name and address, funds awarded to the grantee by HUD and the location of the project; (2) project-level, including the type of housing (whether multi-family, single-family, etc.), the number of units, the number of solar systems to be installed and the square-footage of living area in each building; and (3) systemlevel, including the following items:

*Numbers in brackets pertain to References on page 67. 
- type of system (heating, cooling or domestic hot water),

- kind of system (active, passive or a combination of the two),

- transfer medium used in the solar collector (air or liquid),

- manufacturer of the solar collector,

- aperture area of the solar collector,

- each system's cost (total cost of the operational system and the amount being funded by the government),

- total load required and the amount each solar system will supply to produce heating, cooling and/or domestic hot water.

Updates to Grant file data are periodically submitted to the Solar Data Center. The calendar date for the most recent update for a particular computer listing is shown in the upper left-hand corner of each page of the Grant Reports contained in Section 4. Updated Grant file computer reports can be obtained from:

Franklin Research Center 1030 15th Street, N.W., Suite 170

Washington, D.C. 20005

Attention: Dr. Gerald Mara

Telephone: (202) 223-8109 


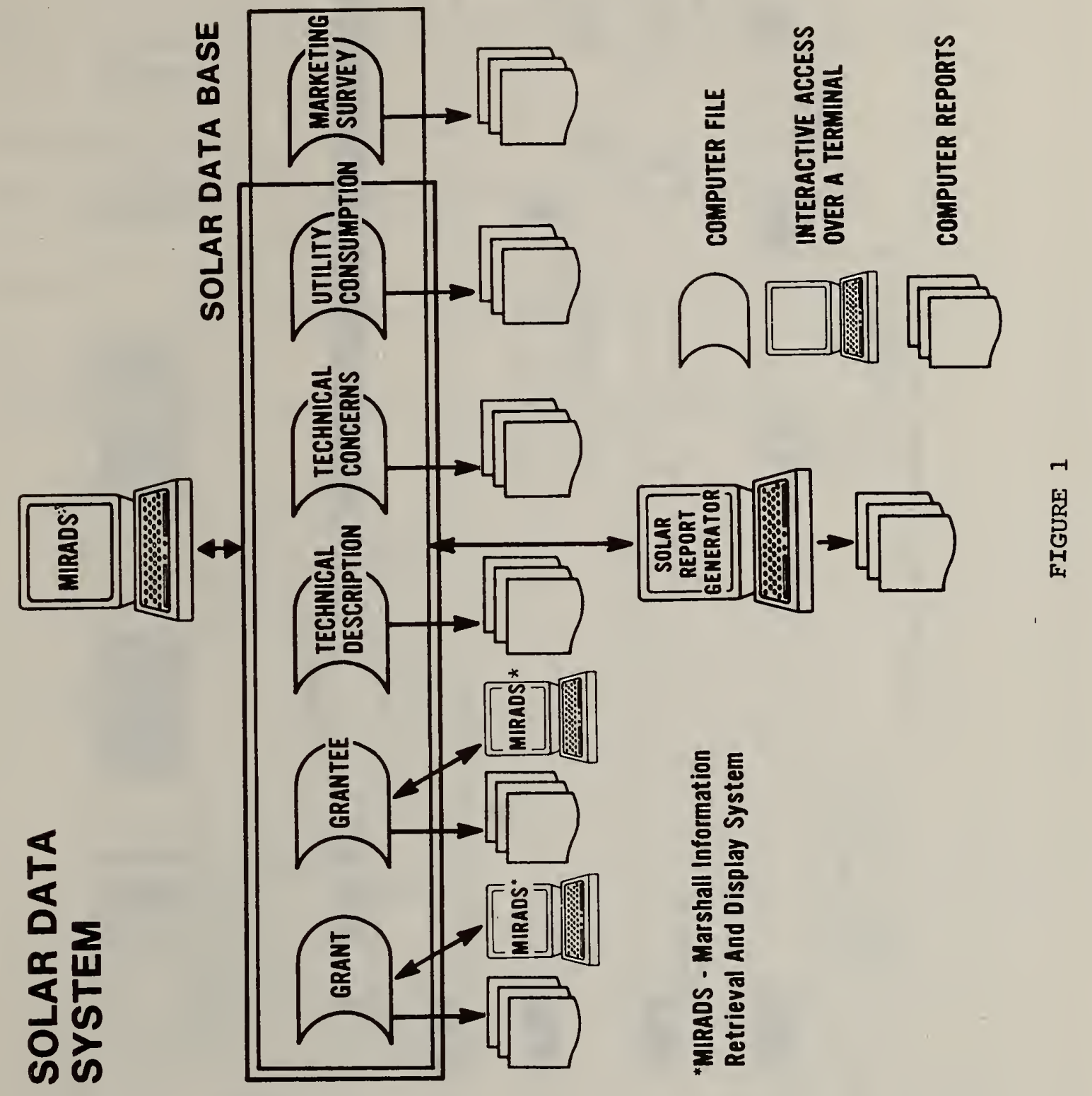


जo
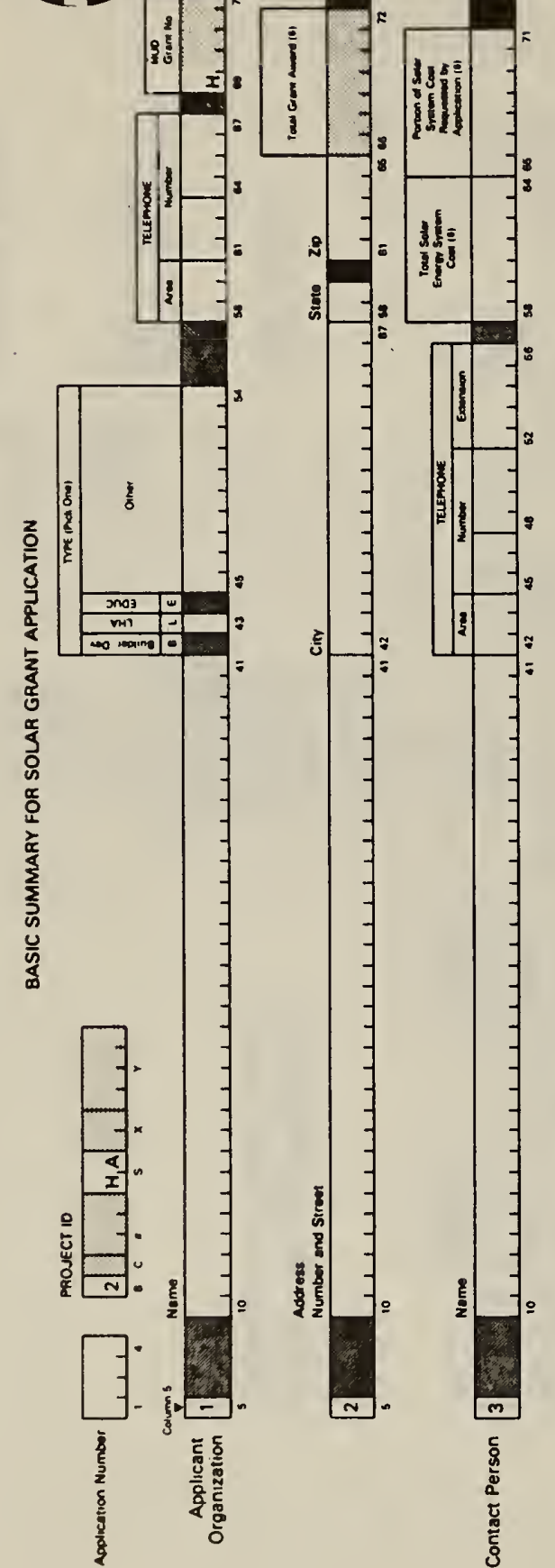
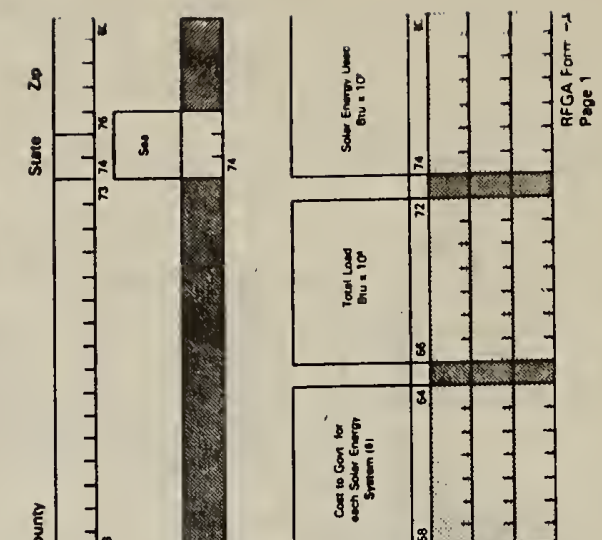

率
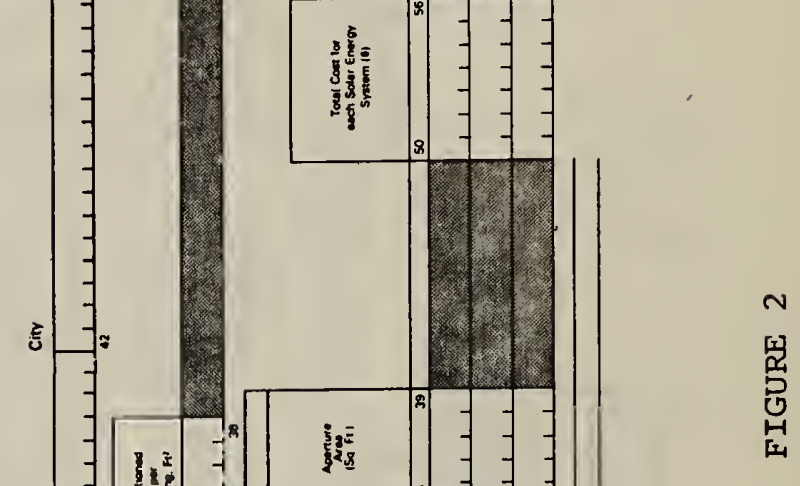
The table in this section sumarizes the data elements which appear in the computer reports contained in section 4 . The following is an explanation of terms used in the column headings of this table:

Descriptive Name: The name given to the data element.

Terms of Measure: When appropriate, the units in which the data are recorded.

Codes:

Codes and their interpretation for those data elements which are coded.

Definition

of Data Element: A full definition of each data item. 
TABLE 1. GRANT FILE DATA ELEMENTS

\begin{tabular}{|c|c|c|c|}
\hline Descriptive Name & $\begin{array}{l}\text { Terms } \\
\text { of } \\
\text { Measure } \\
\end{array}$ & Codes & Definition of Data Element \\
\hline Grantee Name & - & - & $\begin{array}{l}\text { The name of the organi- } \\
\text { zation, firm or person } \\
\text { awarded the HUD grant. }\end{array}$ \\
\hline $\begin{array}{l}\text { Project Address } \\
\text { City and State }\end{array}$ & - & - & $\begin{array}{l}\text { The city and state of the } \\
\text { solar project. }\end{array}$ \\
\hline Grant ID & - & - & $\begin{array}{l}\text { HUD numbering system for } \\
\text { grant awarded. } \\
\text { Cycle } 1 \text { Grant H2423-H2477 } \\
\text { and Grant H2594-H2604 } \\
\text { Cycle } 2 \text { Grant H2700-H2801 } \\
\text { Cycle } 3 \text { Grant H8015-H8212 } \\
\text { Cycle 4 Grant H8301-H8348 } \\
\text { Cycle 4A Grant H8350-H8445 }\end{array}$ \\
\hline $\begin{array}{l}\text { Housing Type } \\
\text { (HOUS TYPE) }\end{array}$ & - & $\begin{array}{l}\text { SFA=single fam- } \\
\text { ily attached } \\
\text { SFD=single fam- } \\
\text { ily detached } \\
\text { MOB=mobile home } \\
\text { GAL=garden } \\
\text { apartment } \\
\text { MFM=multi fam- } \\
\text { ily midrise } \\
\text { MFH=multi fam- } \\
\text { ily highrise }\end{array}$ & $\begin{array}{l}\text { The type of building or } \\
\text { buildings at one location } \\
\text { for a single model within } \\
\text { a grant. A model groups } \\
\text { together buildings with } \\
\text { identical attributes (hous- } \\
\text { ing type, construction } \\
\text { type and size.) }\end{array}$ \\
\hline $\begin{array}{l}\text { Construction } \\
\text { Type } \\
\text { (CNST TYPE) }\end{array}$ & - & $\begin{array}{l}\mathrm{NEW}=\text { new } \\
\mathrm{RET}=\text { retrofit }\end{array}$ & $\begin{array}{l}\text { Whether the system was in- } \\
\text { cluded in a new building } \\
\text { (NEW) or whether an exist- } \\
\text { ing building was retro- } \\
\text { fitted with a solar system } \\
\text { (RET). Used for one lo- } \\
\text { cation, one model in the } \\
\text { grant. }\end{array}$ \\
\hline $\begin{array}{l}\text { Number of } \\
\text { Dwelling Units } \\
\text { (DWELL COUNT) }\end{array}$ & - & - & $\begin{array}{l}\text { Tells the number of dwel- } \\
\text { ling units that are } \\
\text { present in the buildings } \\
\text { at one location, one model } \\
\text { in the grant. }\end{array}$ \\
\hline
\end{tabular}


TABLE 1. GRANT FILE DATA ELEMENTS (Continued)

\begin{tabular}{|c|c|c|c|}
\hline Descriptive Name & $\begin{array}{c}\text { Terms } \\
\text { of } \\
\text { Measure }\end{array}$ & Codes & Definition of Data Element \\
\hline $\begin{array}{l}\text { Number of } \\
\text { Buildings } \\
\text { (NBR BLDGS) }\end{array}$ & - & - & $\begin{array}{l}\text { This is the number of } \\
\text { buildings at one location } \\
\text { and in one model. }\end{array}$ \\
\hline $\begin{array}{l}\text { Number of } \\
\text { Solar Systems } \\
\text { (SOLAR SYS) } \\
\end{array}$ & - & - & $\begin{array}{l}\text { Total number of solar } \\
\text { energy systems at one lo- } \\
\text { cation and in one model. }\end{array}$ \\
\hline $\begin{array}{l}\text { System Type } \\
\text { (SYS TYPE) }\end{array}$ & - & $\begin{array}{l}\mathrm{H}=\text { heating } \\
\mathrm{C}=\text { cooling } \\
\mathrm{W}=\text { hot water }\end{array}$ & $\begin{array}{l}\text { Indicates the applica- } \\
\text { tion(s) of the solar } \\
\text { energy system for the } \\
\text { given system number. }\end{array}$ \\
\hline $\begin{array}{l}\text { System Kind } \\
\text { (SYS KIND) }\end{array}$ & - & $\begin{array}{l}\mathrm{A}=\text { active } \\
\mathrm{P}=\text { passive } \\
\mathrm{H}=\text { hybrid }\end{array}$ & $\begin{array}{l}\text { Tells whether the system } \\
\text { is active, passive or } \\
\text { hybrid. }\end{array}$ \\
\hline $\begin{array}{l}\text { Manufacturer } \\
\text { of Solar } \\
\text { System } \\
\text { (SOLAR } \\
\text { MANUFACTURER) }\end{array}$ & - & - & $\begin{array}{l}\text { Name of the collector/ } \\
\text { solar system manufac- } \\
\text { turer of the given system. }\end{array}$ \\
\hline $\begin{array}{l}\text { Collector Area } \\
\text { (aperture area) } \\
\text { (CLTR SQFT) } \\
\end{array}$ & Sq.Ft. & - & $\begin{array}{l}\text { The gross aperture area of } \\
\text { the collector array used } \\
\text { in the solar system. }\end{array}$ \\
\hline $\begin{array}{l}\text { Solar System } \\
\text { Cost to } \\
\text { Government } \\
\text { (CosT To GOV) }\end{array}$ & $\$$ & - & $\begin{array}{l}\text { The cost to HUD (amount } \\
\text { funded) for the solar } \\
\text { system. }\end{array}$ \\
\hline $\begin{array}{l}\text { Solar System } \\
\text { Cost per MBtu } \\
\text { (COST/MBTU) }\end{array}$ & $\$$ & - & $\begin{array}{l}\text { The total cost of one of } \\
\text { the solar systems divided } \\
\text { by the solar energy (in } \\
\text { MBtu) supplied (per year) } \\
\text { by the solar system. The } \\
\text { MBtu value is predicted by } \\
\text { the applicant. }\end{array}$ \\
\hline
\end{tabular}




\section{GRANT FILE COMPUTER REPORTS}

Grant file data stored on the computer can be retrieved in two ways: (1) by several formatted batch computer reports, which present the data elements in a variety of sorted orders; and (2) through an interactive query system, which gives the user at a remote terminal the flexibility of specifying the context and format of the report. This document addresses the formatted batch computer reports that are available. A separate publication, User's Guide for online Retrieval of Solar Data Using MIRADS [3], contains user instructions for online access of the Grant file, a service which is available to users authorized by HUD (primarily Demonstration Program contractors).

There are eight computer reports available using Grant data. Four of these reports are contained in this document. The following is a description of all eight of these computer reports and an indication of which four are being included herein.

HA-Cl: ${ }^{*}$ Detailed Listing of Solar Grants

This report contains a listing of all elements of data which are entered into the database for each grant awarded by HUD. These data elements consist of information about the grant, the location of the project, the building(s) which will be newly constructed or "retrofitted" with a solar energy system and the solar energy system(s) itself. This report contains over 250 pages of computer printout and is not included here. However, the report is available from the Franklin Research Center at the address shown on page 2 .

HA-C2: Units and Cost Analysis

This printout is a one-page summary of the Grant file data broken down by type of building (single family detached, multi-family detached, etc.), type of solar system configuration (hot water only, heating and cooling, etc.) and type of construction (new or retrofit). These data are summarized by the number of dwelling units, by cost to HUD for the grant and by the average cost of a dwelling unit. summary counts are given for number of solar systems, number of buildings and number of active, passive and hybrid systems. This report is shown in section 4.1 , pages 11-12.

\footnotetext{
* Each computer report has a number associated with it. The number or the name of the report can be used when requesting copies.
} 
This approximately 15-page report presents selected data on all HUD grants. Fourteen of the most important data elements for each project are incorporated in this report, including: identification of the grantee; the location of the project; housing type (single family detached, multi-family detached, etc.); construction type (new or retrofit); counts of units, systems and buildings; basic system description, including collector manufacturer; and cost to HUD for the grant. Because grant numbers were assigned in sequence by HUD, the HA-C3 report lists grant Cycle 1 data before grant Cycle 2 data, etc. Trends in the kinds of projects which were emphasized in the funding process (e.g., by types of systems used - whether heating, cooling or hot water) are more apparent by scanning this report than any of the others. This report is shown in Section 4.2, pages 13-26.

HA-C4BC: Summary of Solar Grants Sorted on Manufacturer With Cost per MBtu

This report, which is sorted on solar collector manufacturer, contains the same data elements as the HA-C3 report described above, except that the square-footage of collector area and cost per MBtu are shown instead of the cost to HUD. This report is shown in section 4.3, pages 27-45.

\section{HA-C4CG: Summary of Solar Grants Sorted on Manufacturer}

This report, which is sorted on solar collector manufacturer, contains the same data elements as the $\mathrm{HA}-\mathrm{C} 3$ report described above, except that the square-footage of collector area is shown instead of the cost to HUD. This report is not included in this document.

\section{HA-C5AS: Summary of Solar Grants Sorted on Grantee City and state}

This report also contains essentially the same data elements as the $\mathrm{HA}-\mathrm{C} 3$ report described above, except data are sorted by grantee city and state. Also the location of each grantee is printed instead of the corresponding location of the project. The states are usually the same, but the cities may differ. This report is not included in this document. 
HA-C5PS: Summary of Solar Grants Sorted on Project

City and state

This report, which is sorted by project city and state contains the same data elements as the HA-C3 report described above. This report is shown in section 4.4, pages $46-62$.

HA-C7: Summary of Solar Grants Sorted and Totaled by Project HUD Region

This report, which is sorted by HUD geographical region, contains the same data elements as the HA-C3 report described above with the addition of the appropriate region code for each grant. This report is not included in this document.

As previously noted, updated copies of the computer reports included in this publication as well as copies of the Grant file computer reports not included here may be obtained from the Franklin Research Center. (See page 2.) 
4.1 UNITS AND COST ANALYSIS 


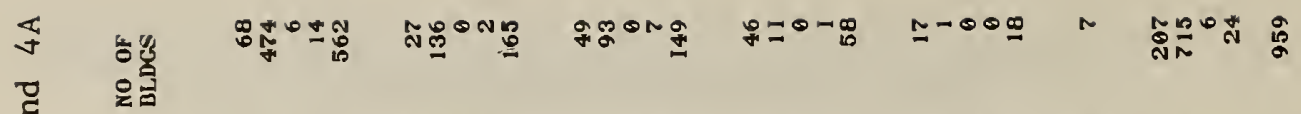

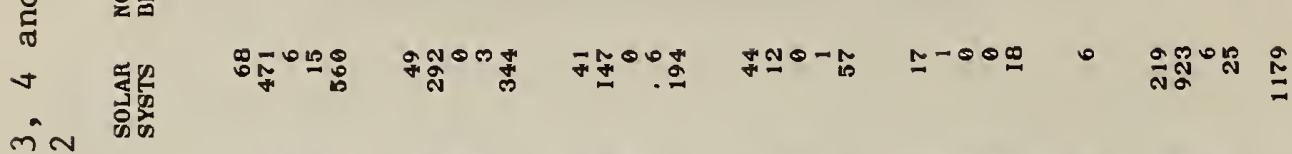

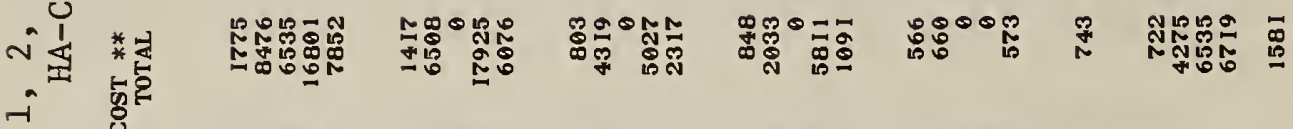

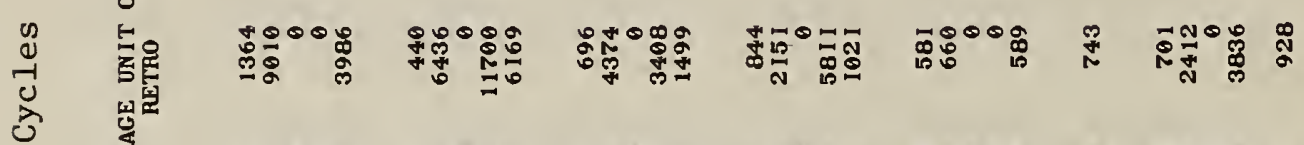

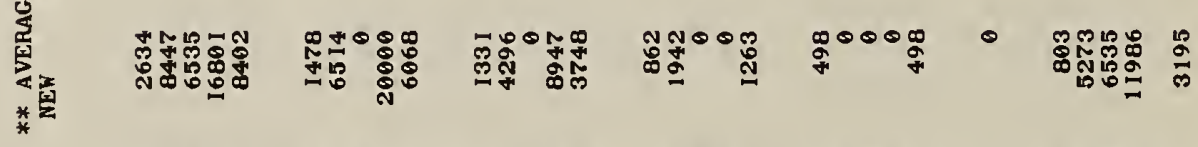

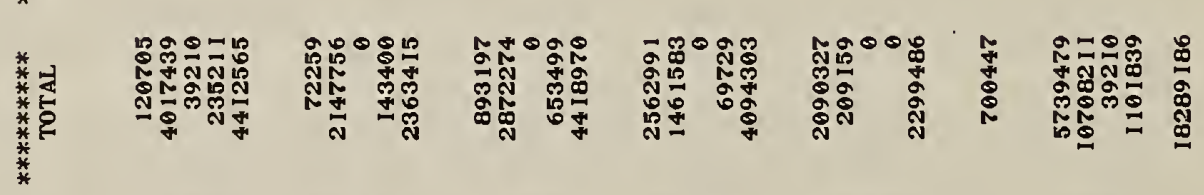

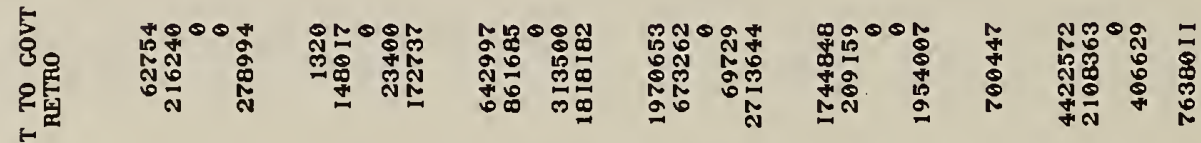

ते

安

1
0
0

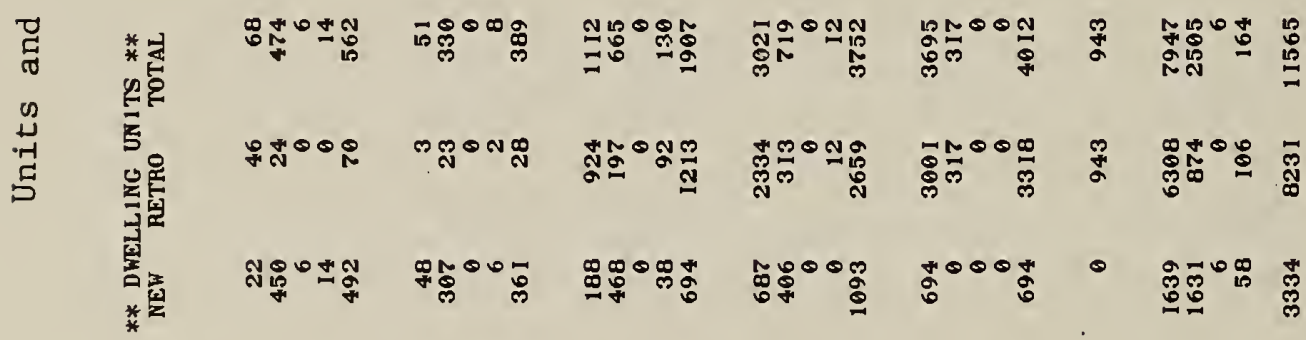

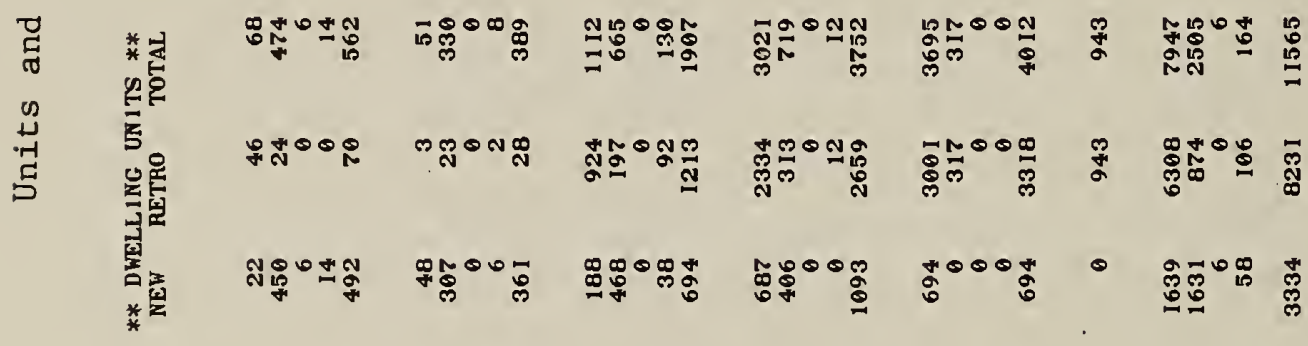

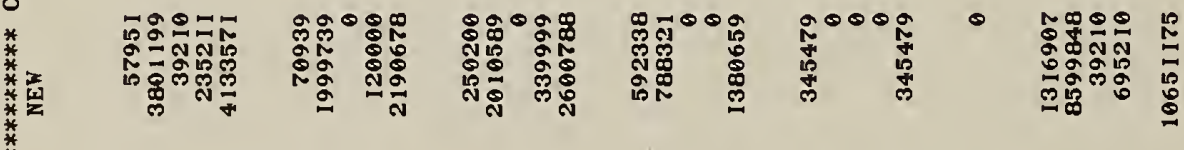

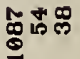

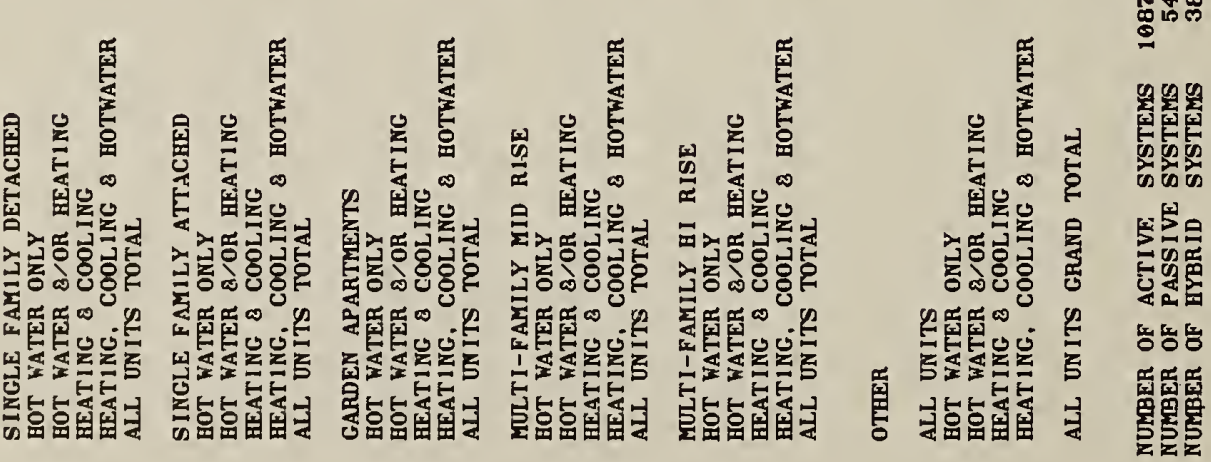




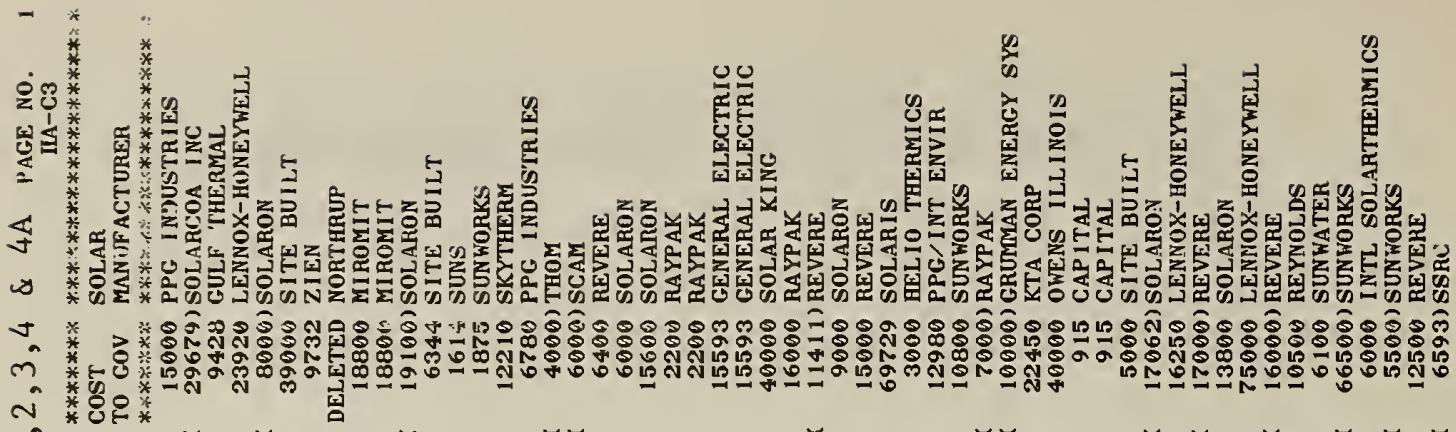

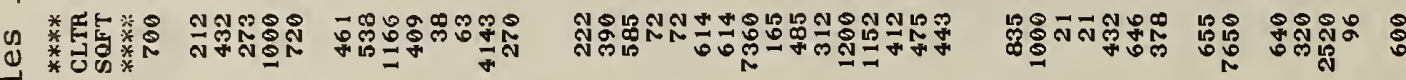

$\lambda$

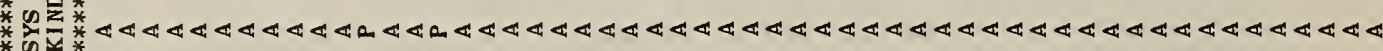

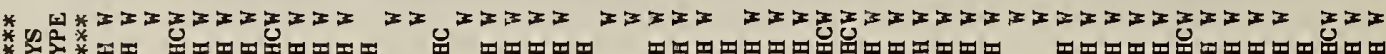

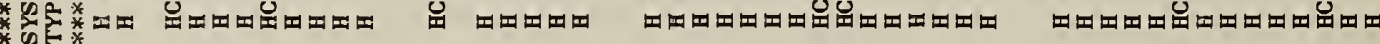
*

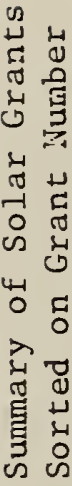

*

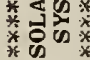

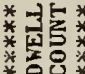

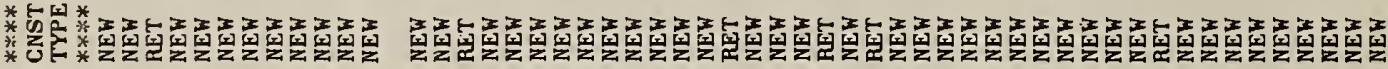

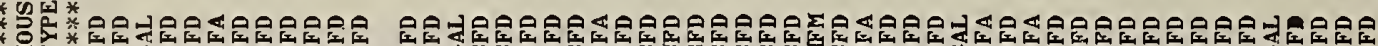

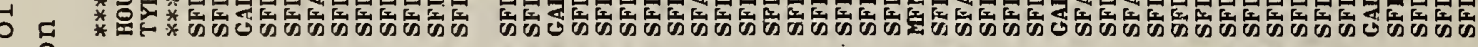

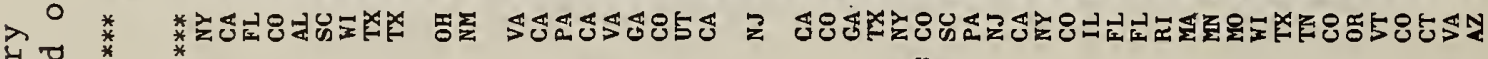

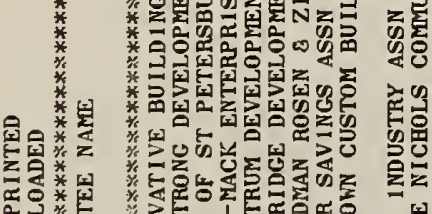

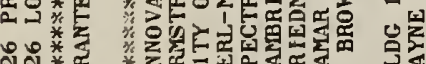

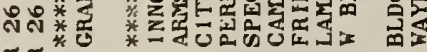

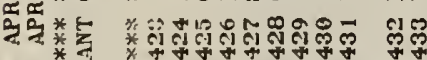
2: $2:$ :
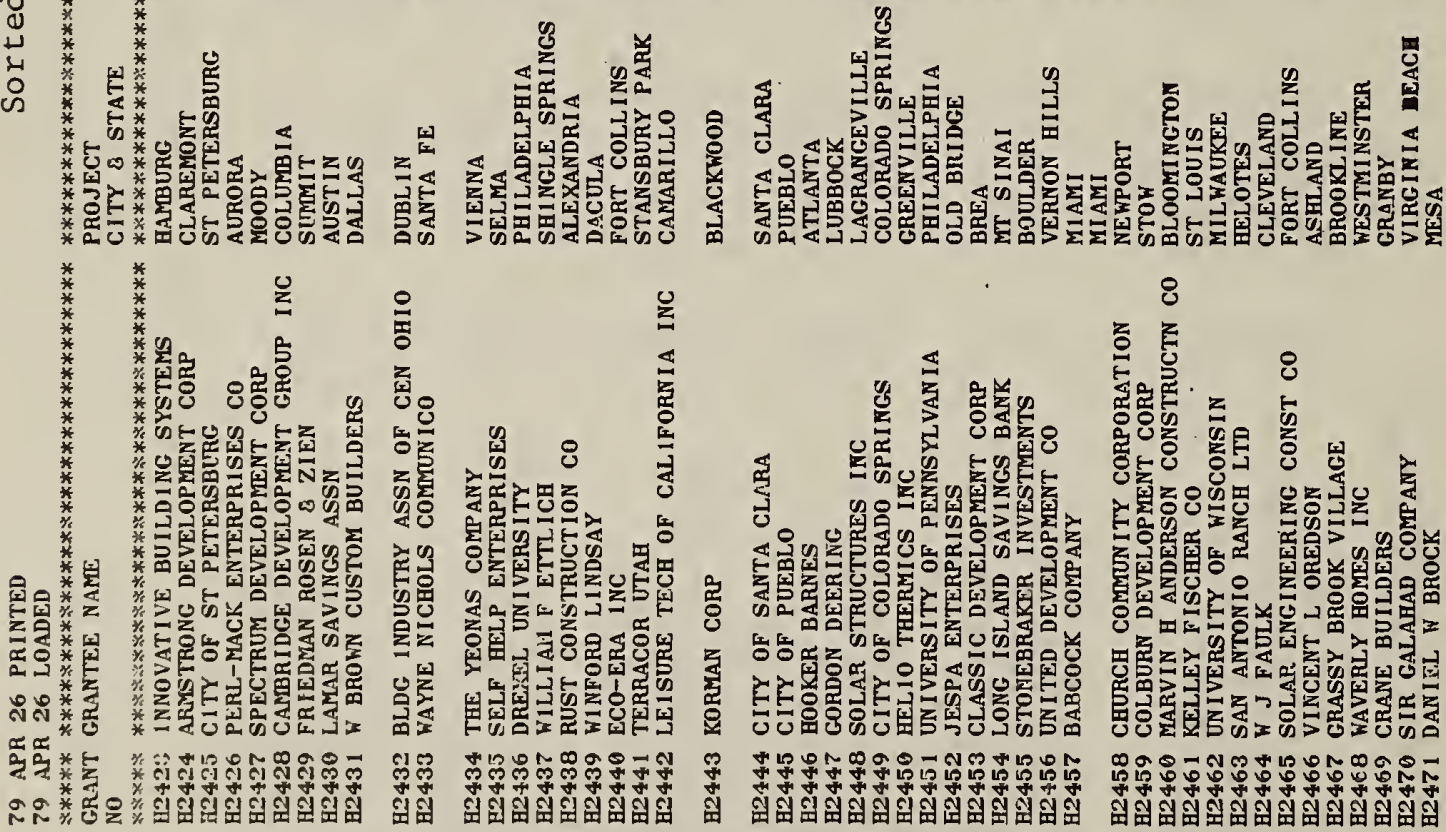


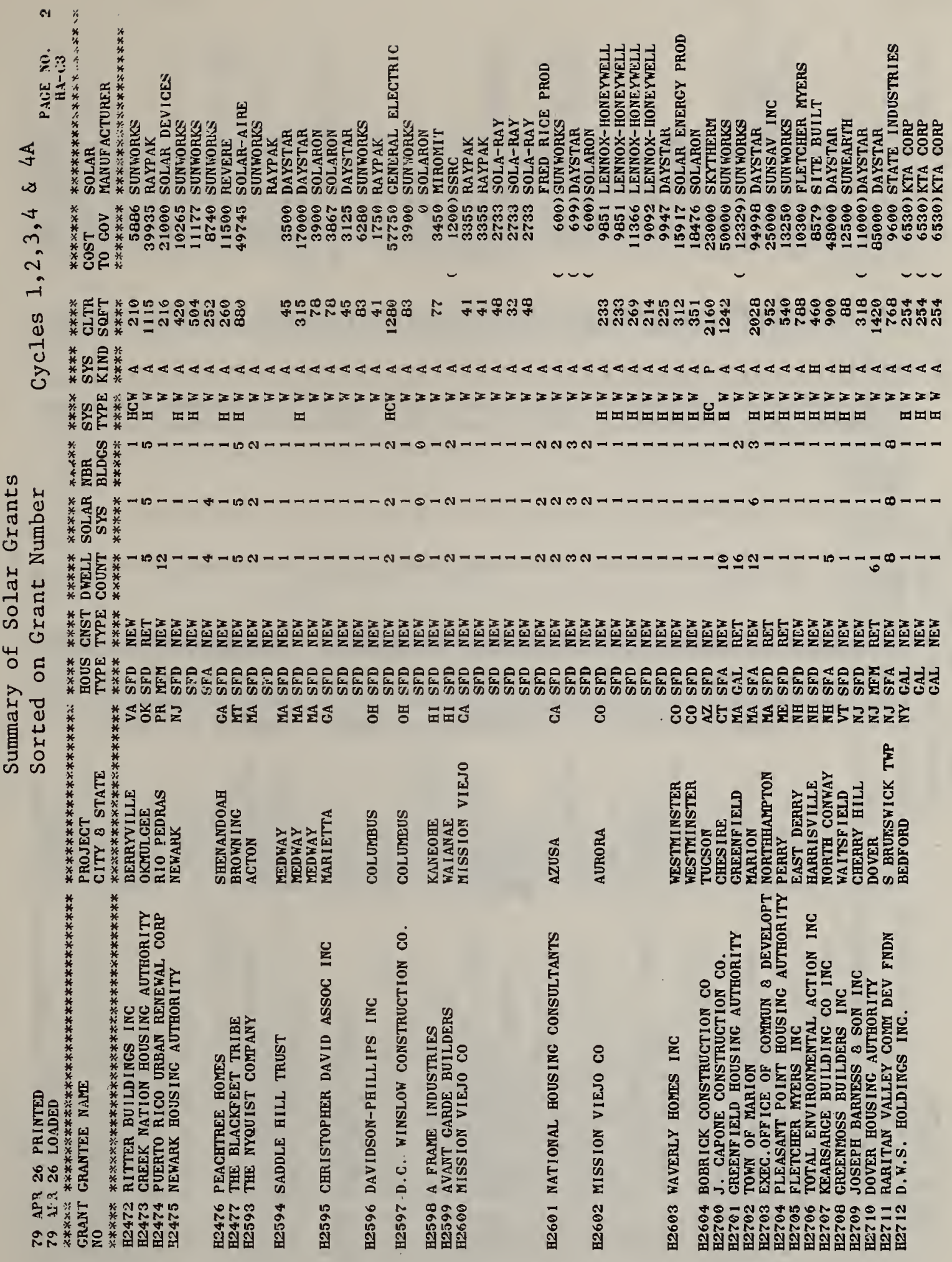




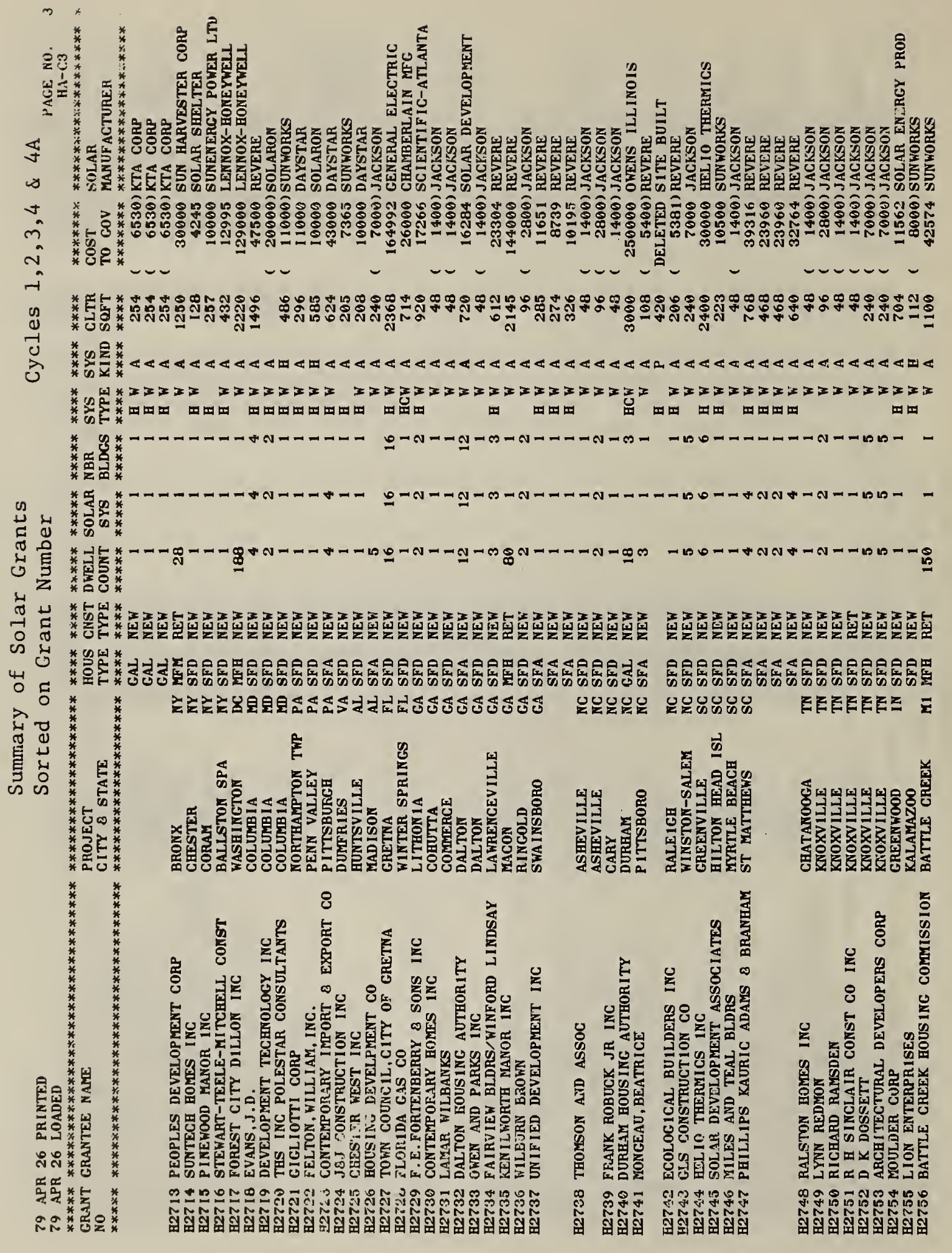




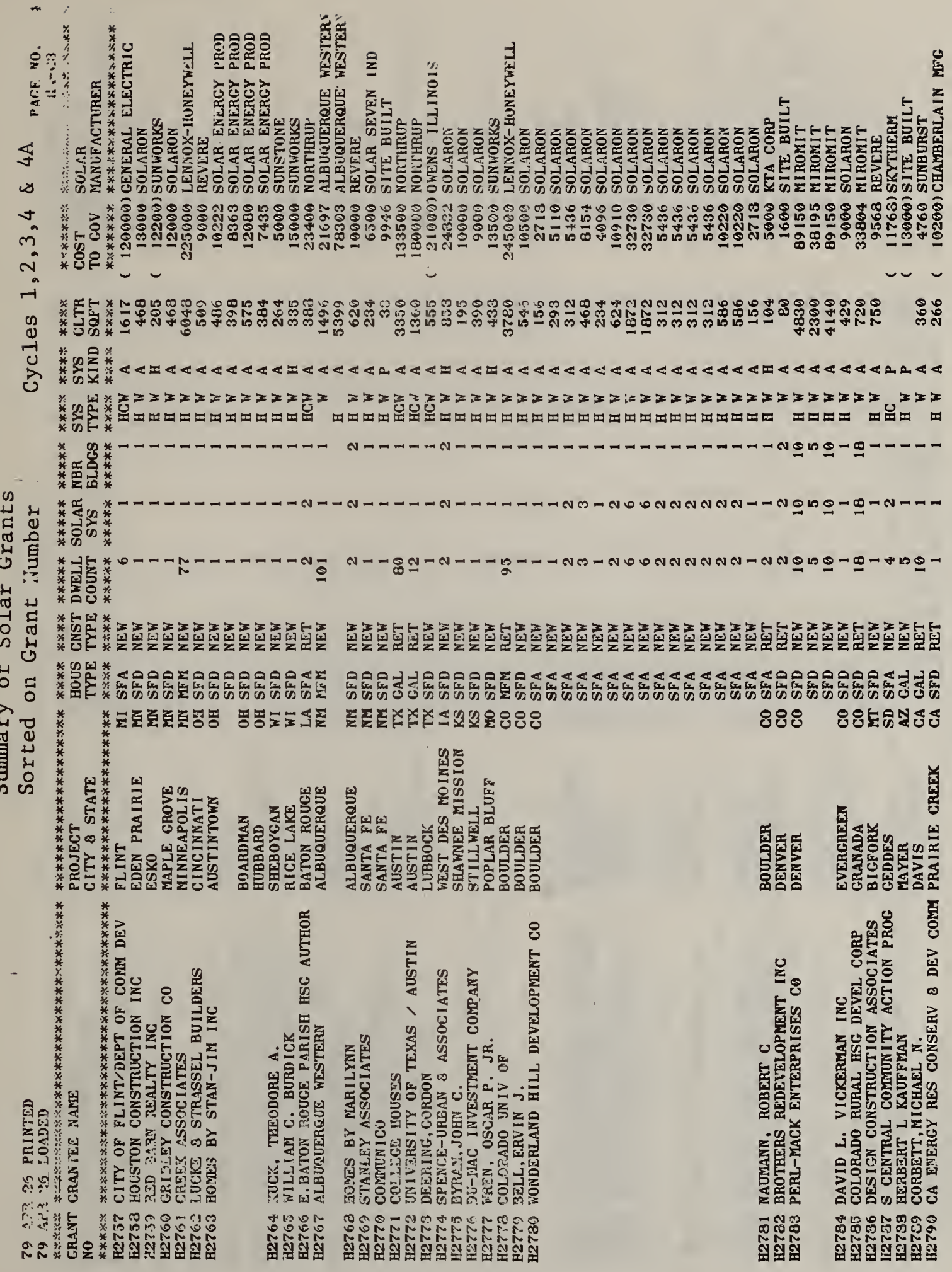




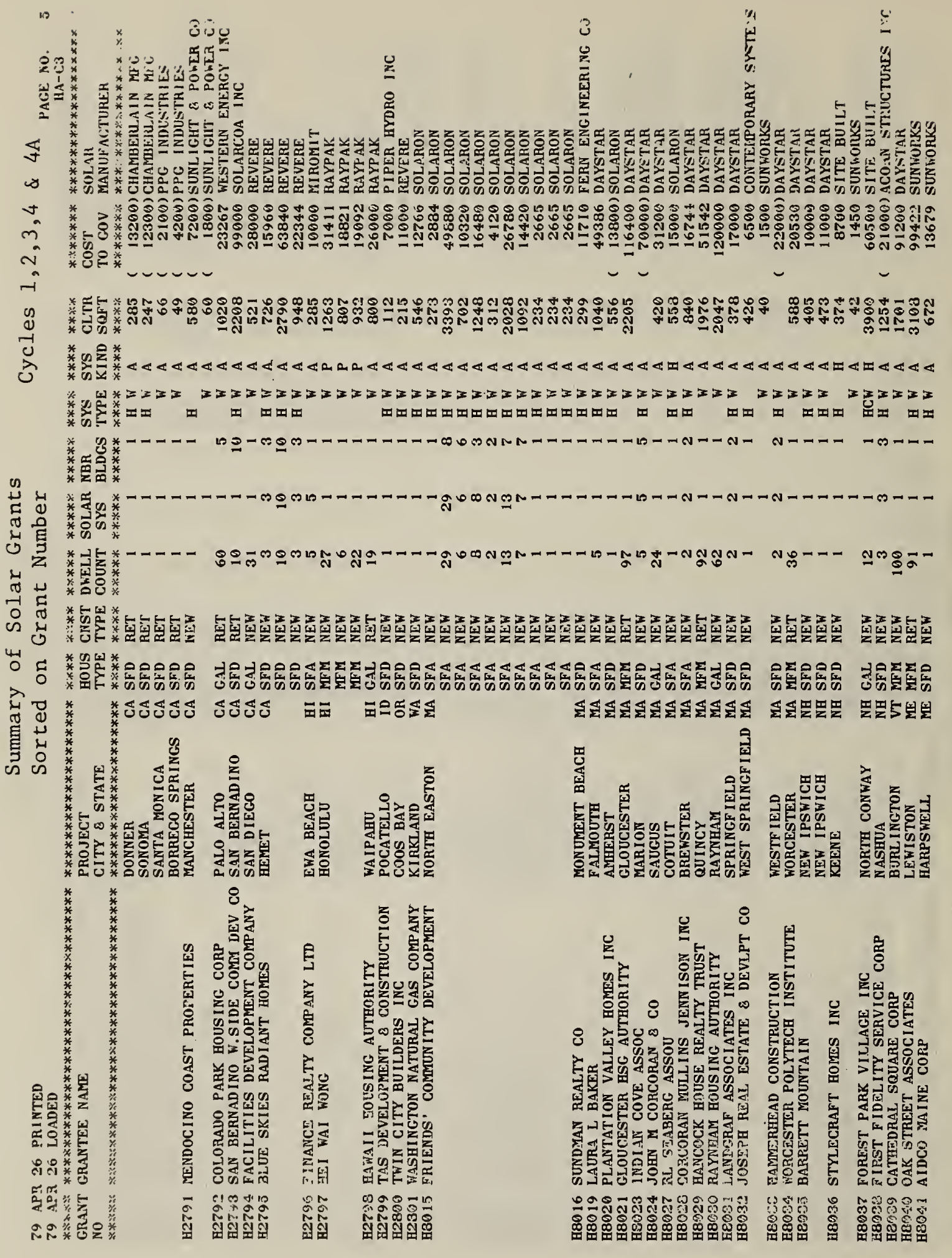




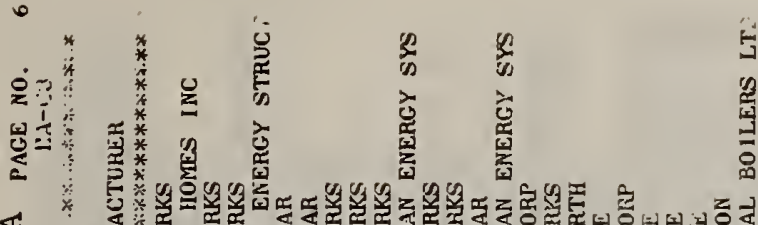

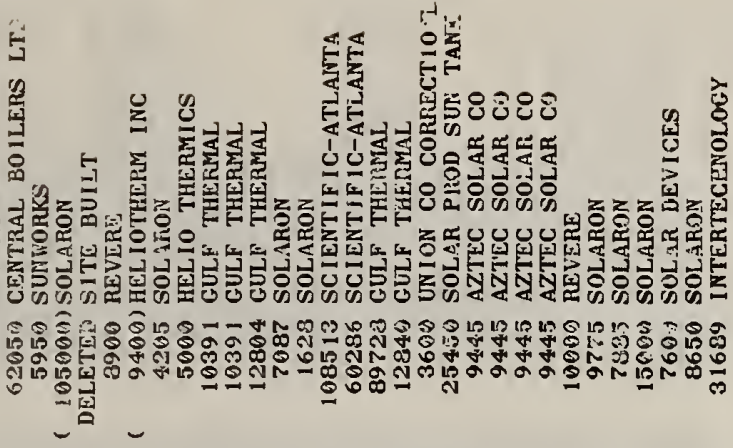

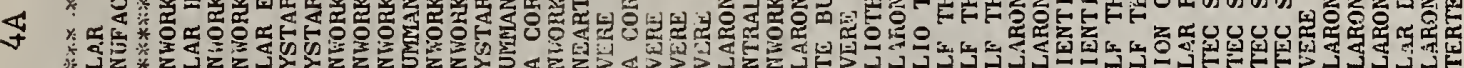

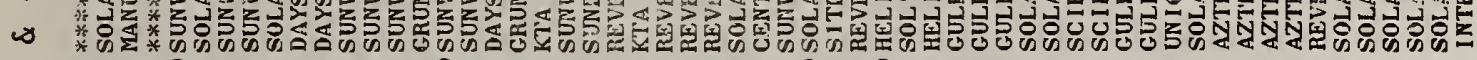

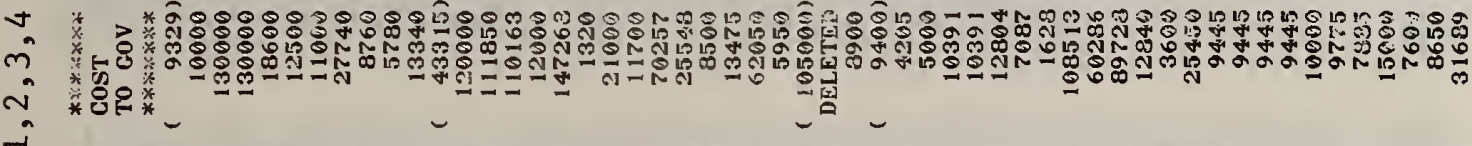

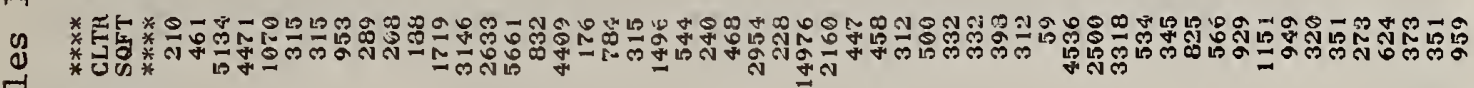

U.

它

*

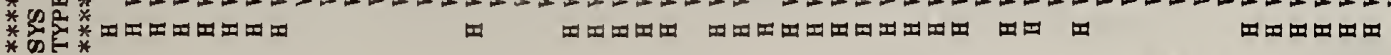

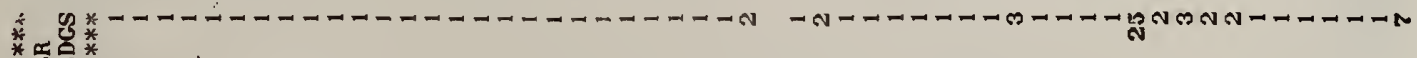

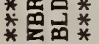

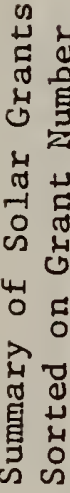

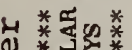

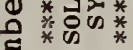

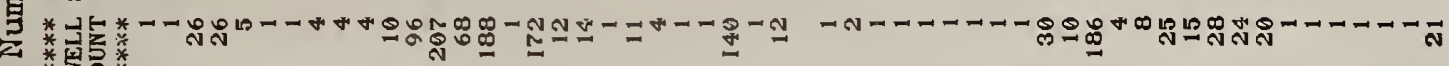

事言获

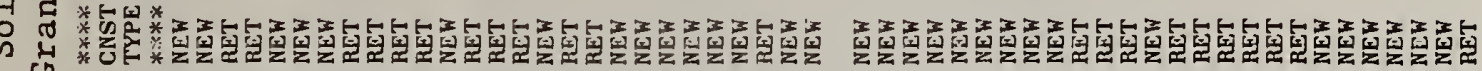

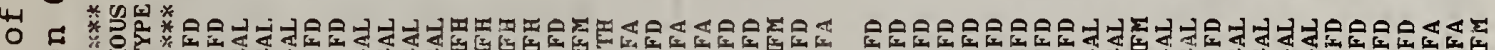

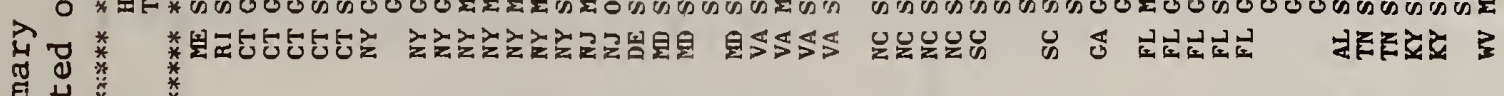

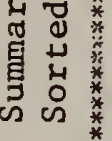

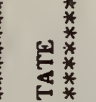

놀

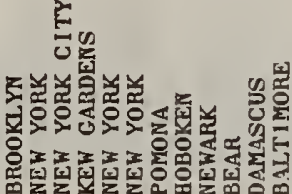

*

幽四

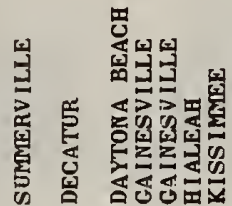

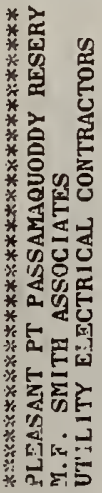

3

헐

둔

号

客

$\checkmark$

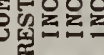

융 흥

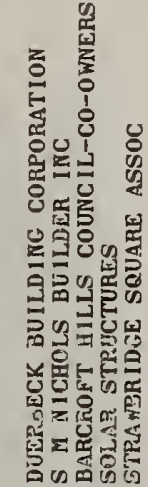

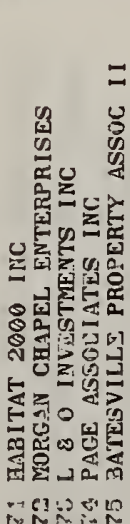

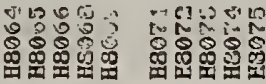

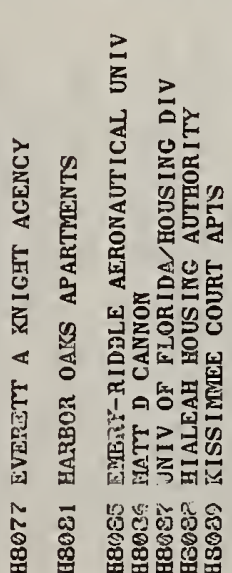

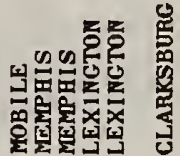

छ

8

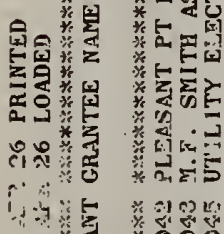

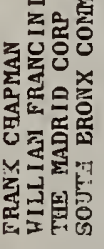

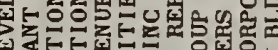
苗

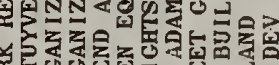
ब的 tri

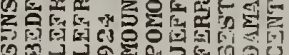

0 군

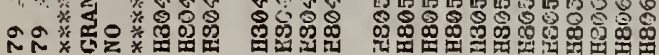

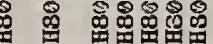

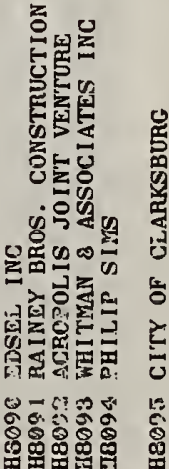




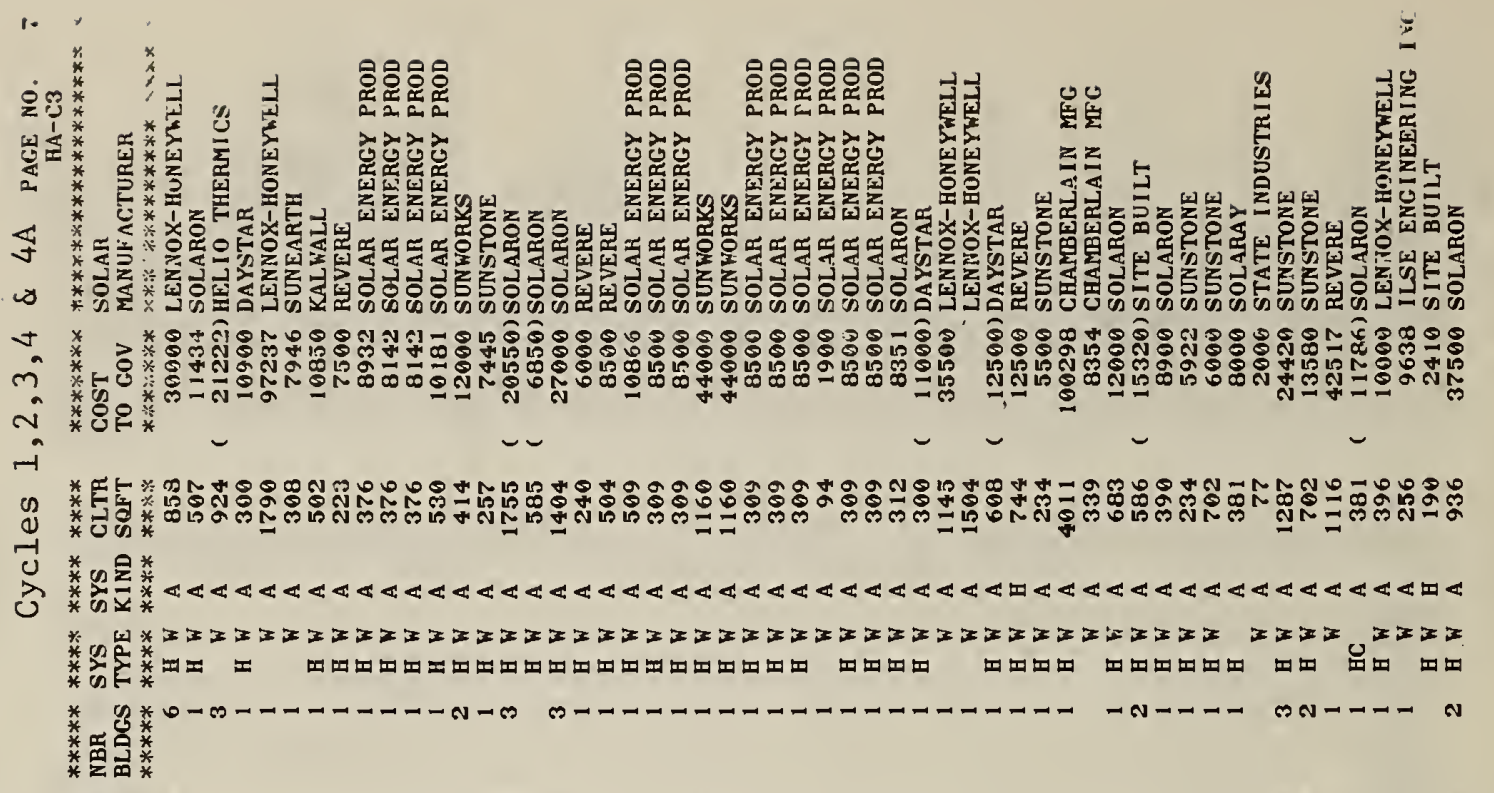
只

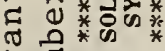

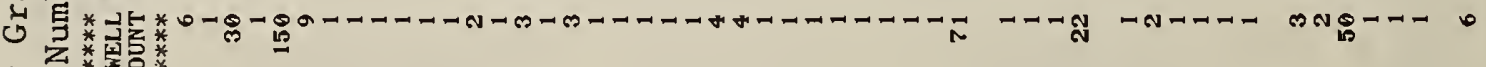

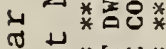

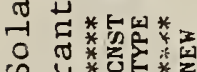

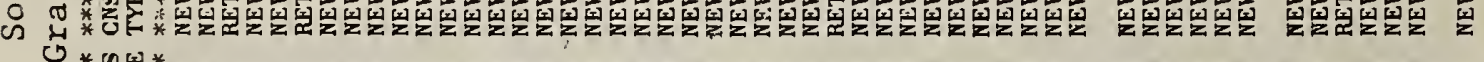
फ * *

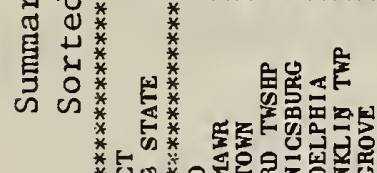

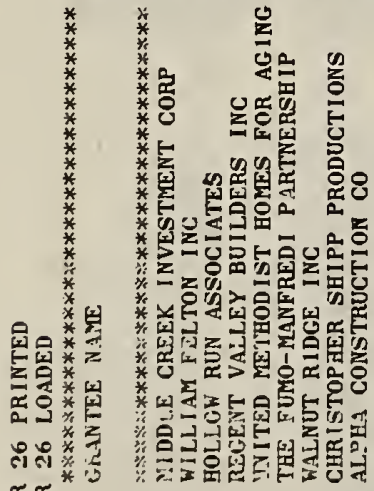

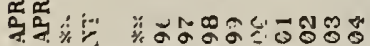

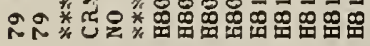
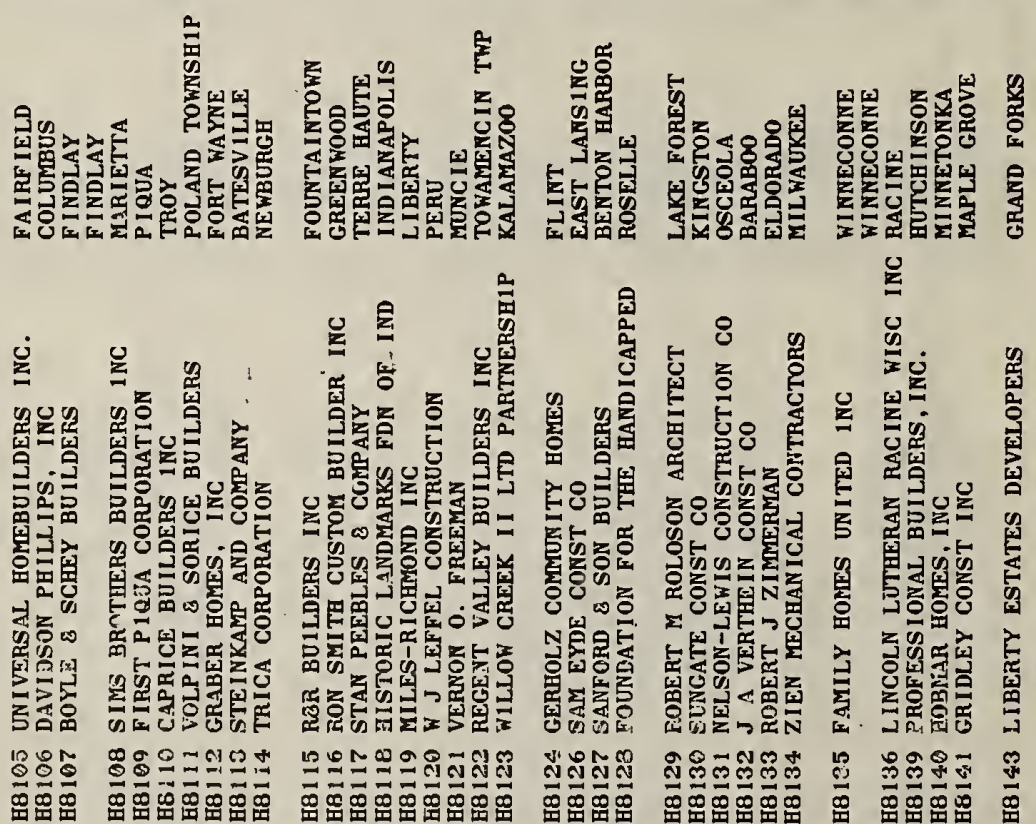

$\mathrm{\Xi}$

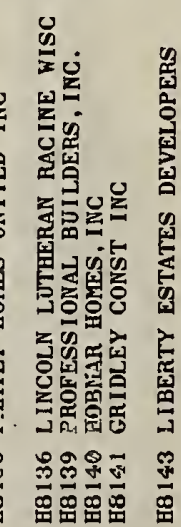




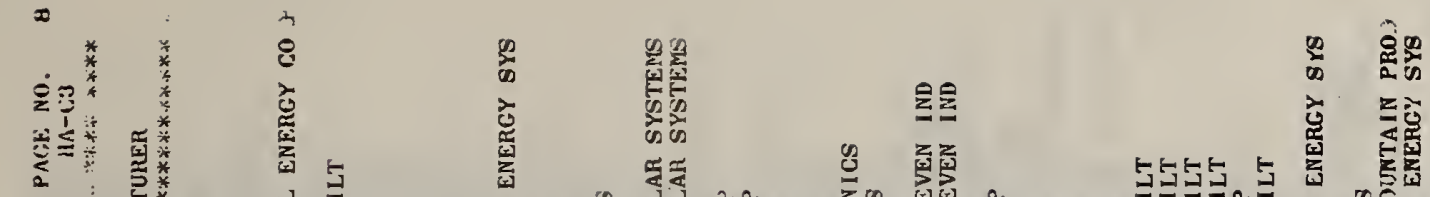

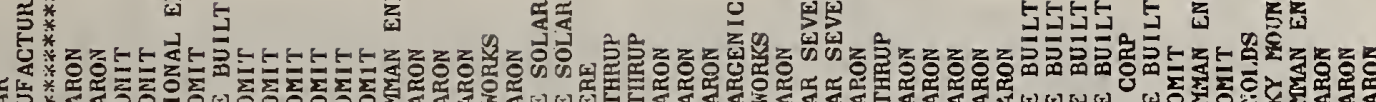

\ : 준

3.

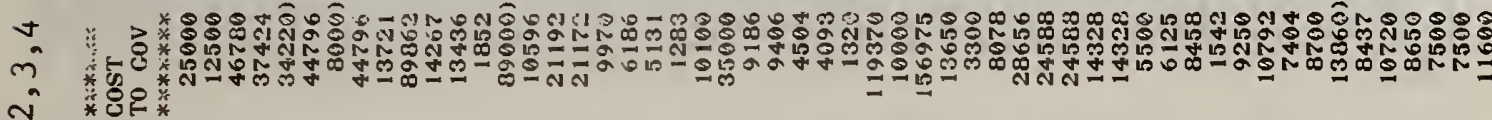

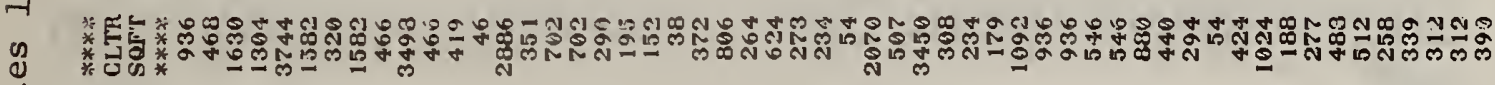

ठे

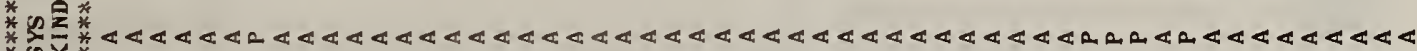

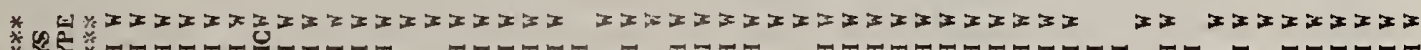

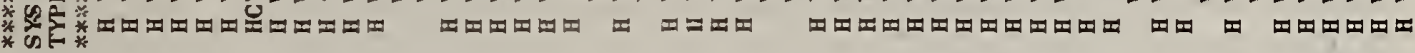

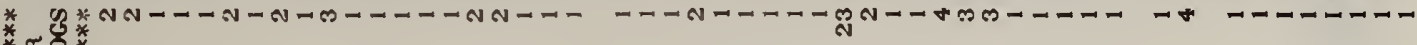
*

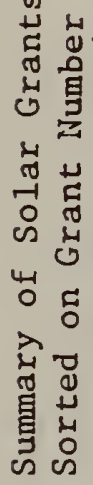

*

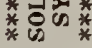

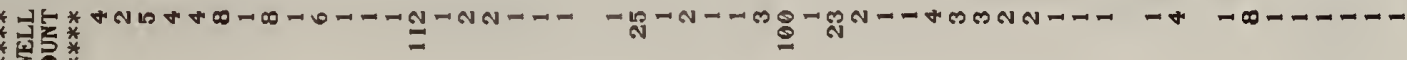
*

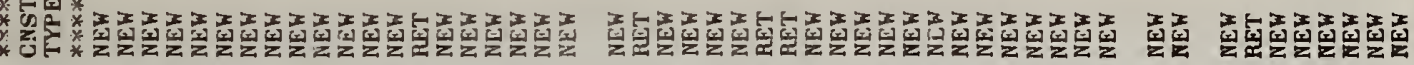

댕

क क क 7 *

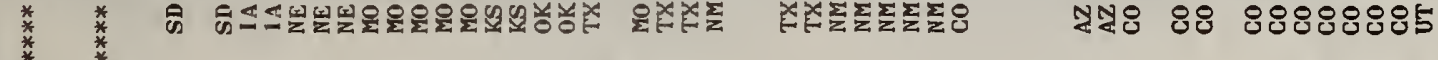

*

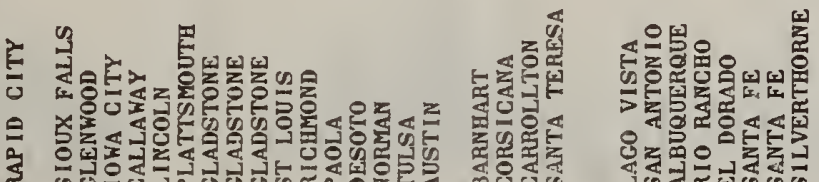

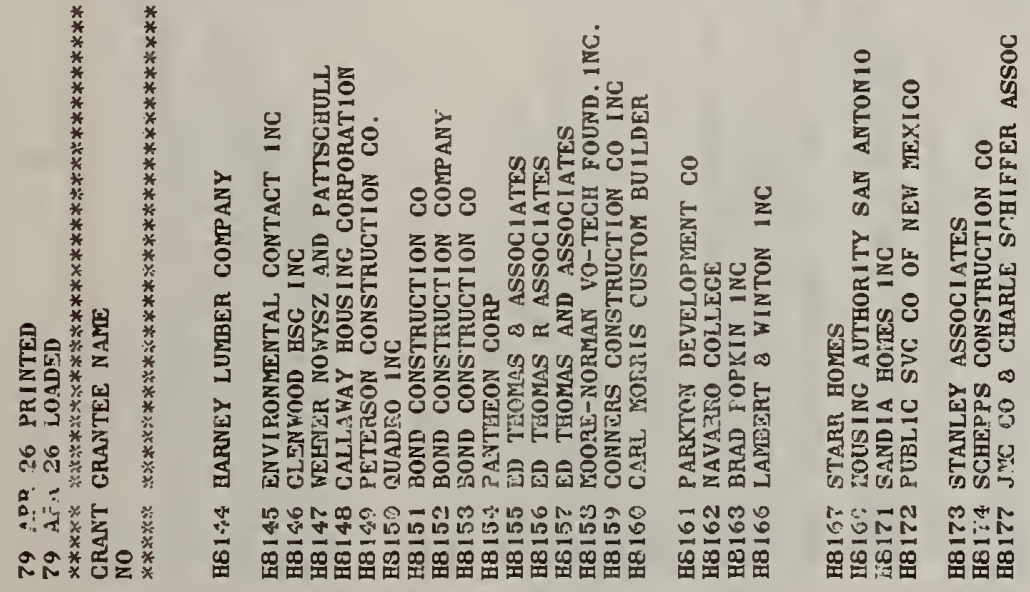

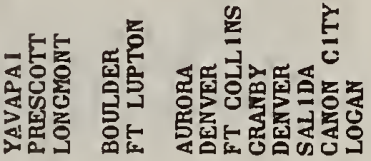




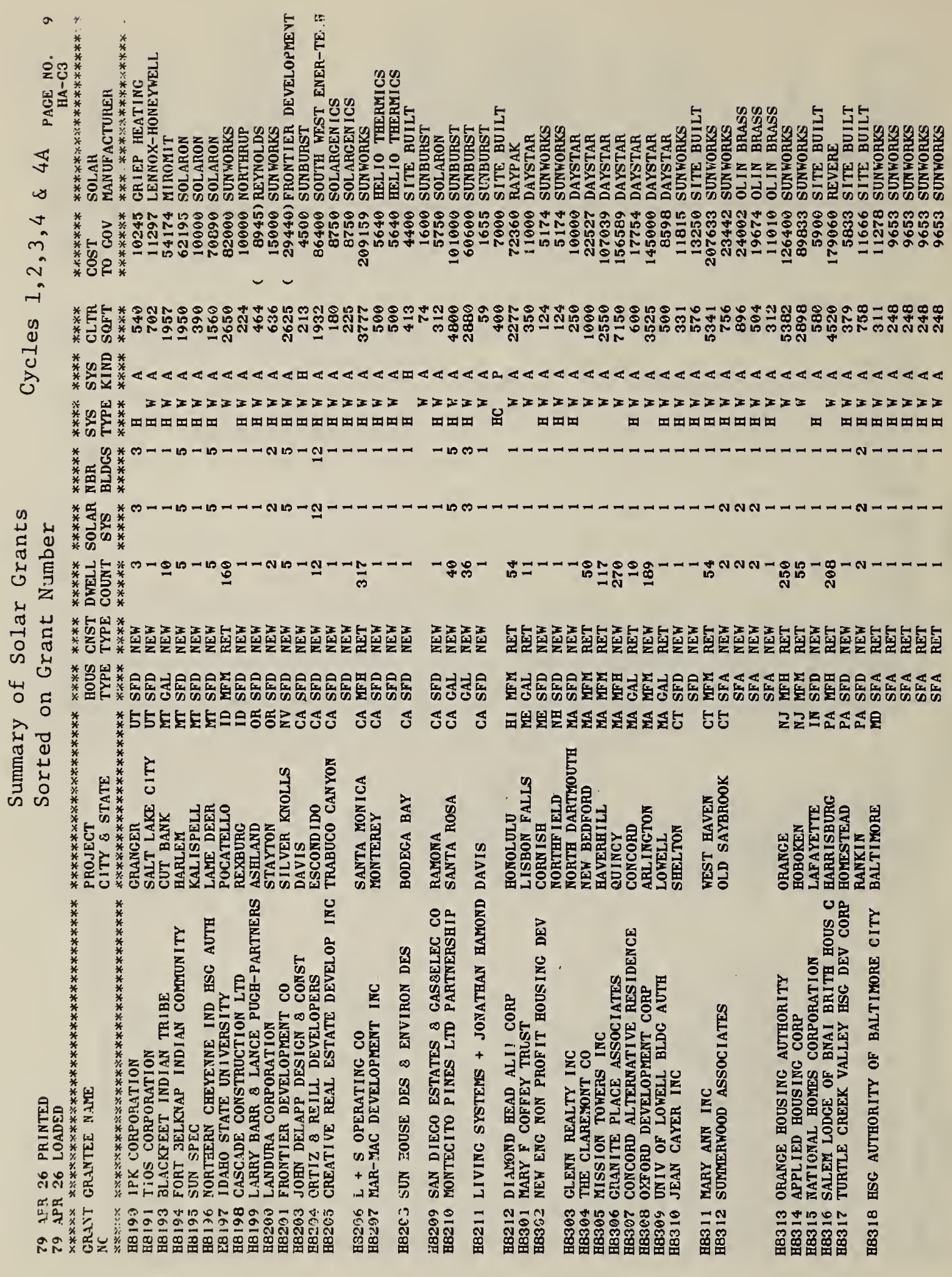




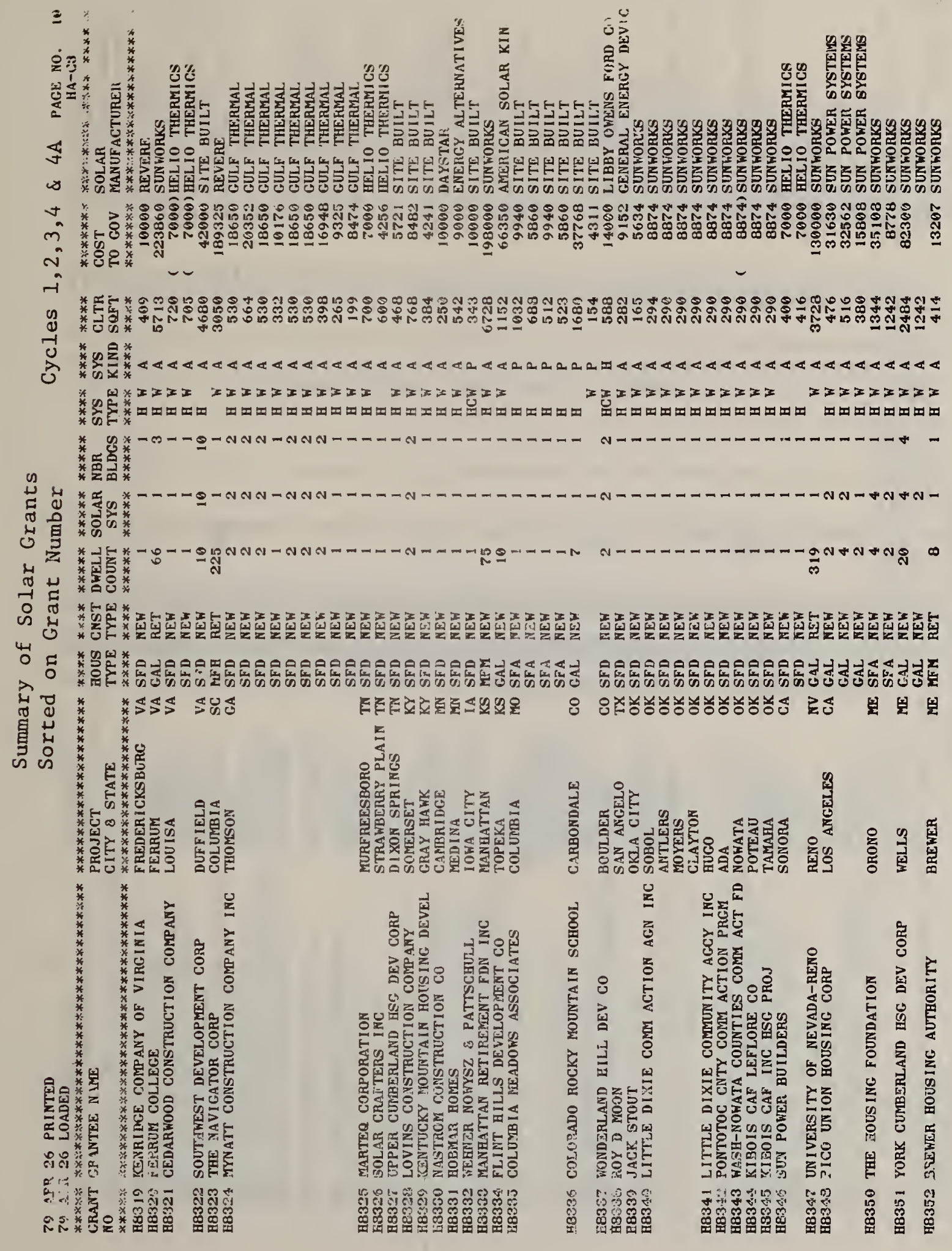




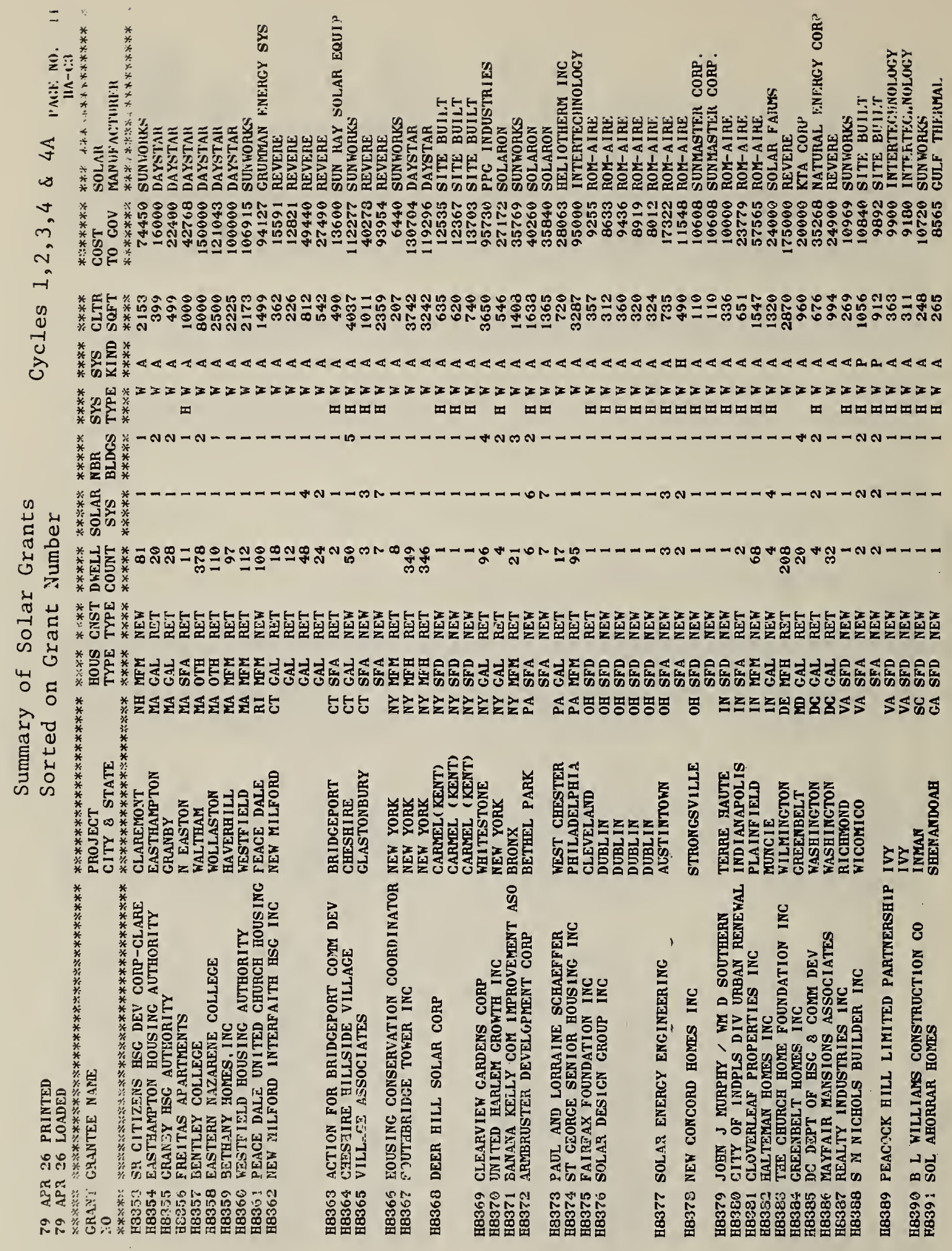




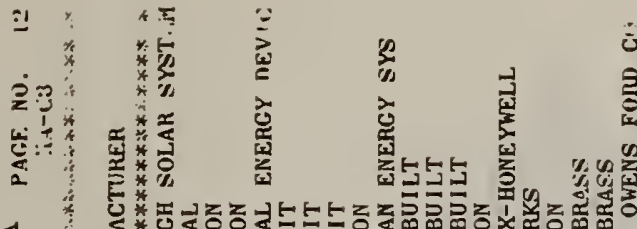

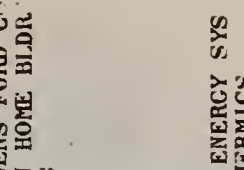

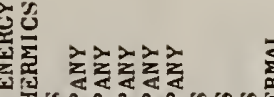

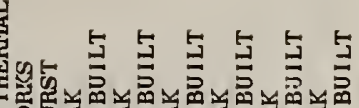

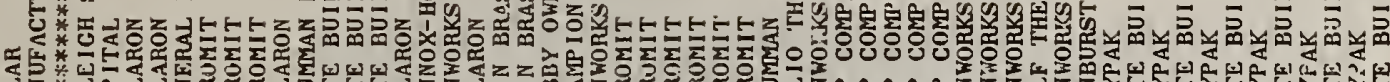

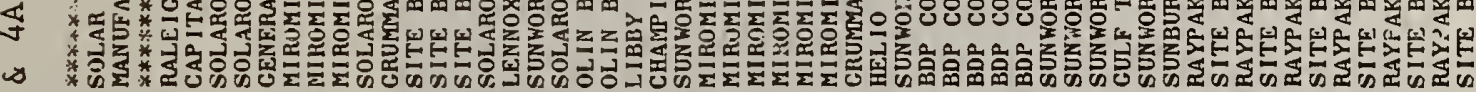

士

i *

$-1$

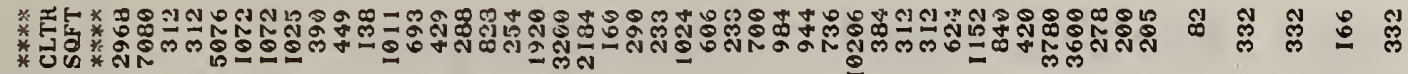

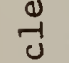
*

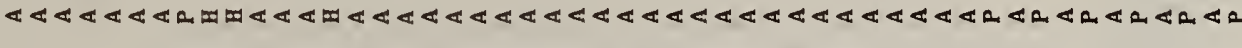

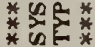

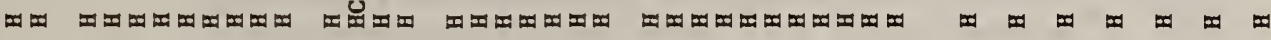
*

a

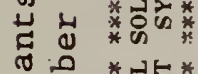

它

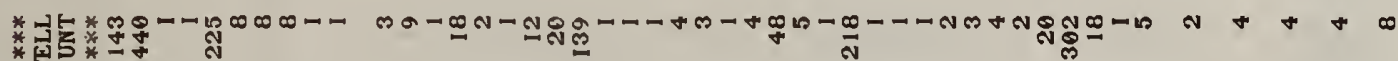

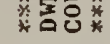

(⿻)

is

岩

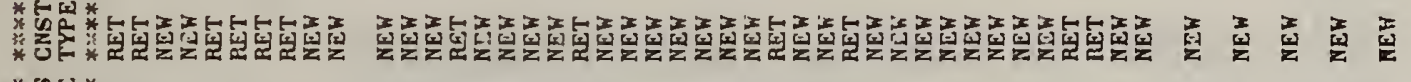

*

高总

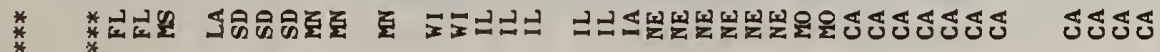

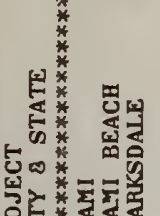

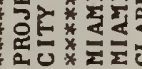
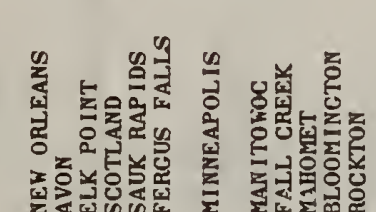

息

空

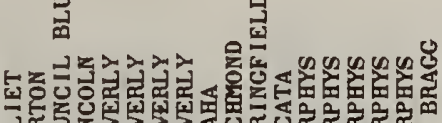

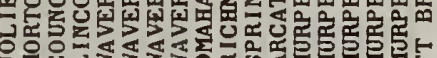

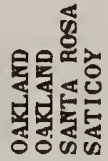

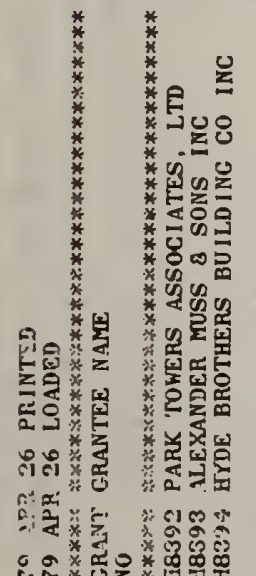
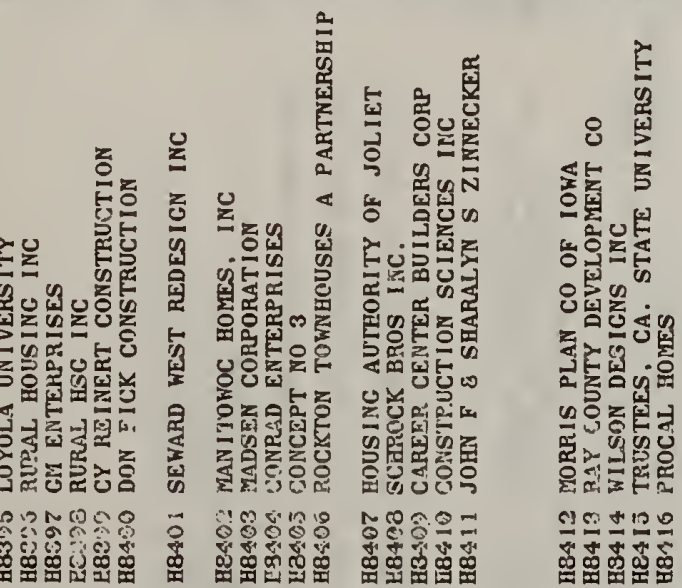

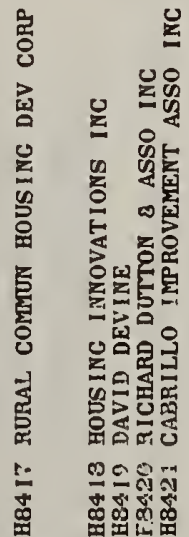




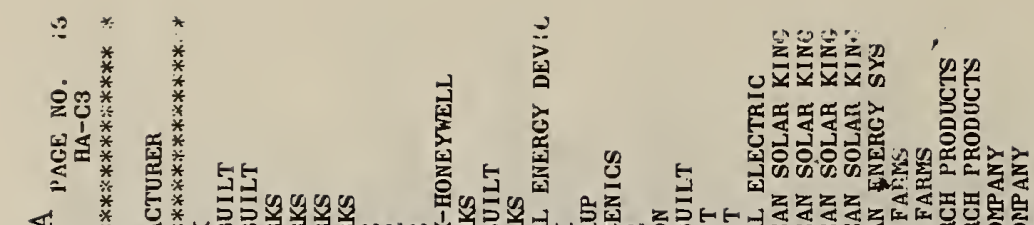

广 * * * * *

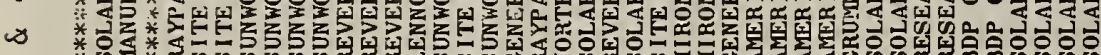

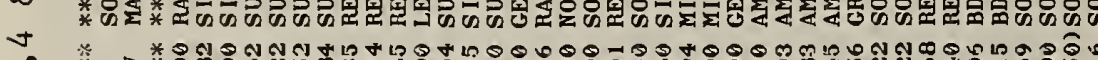

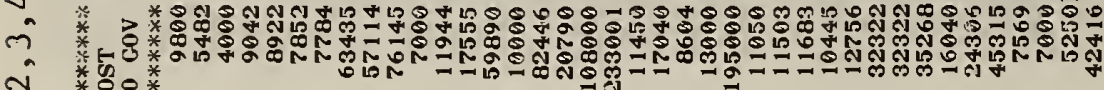

ก.

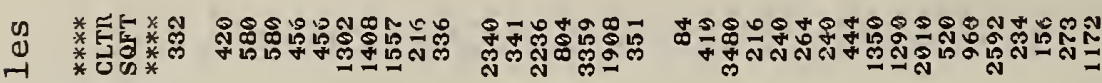

J

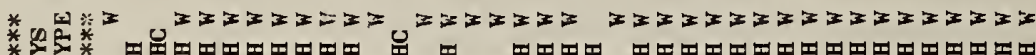

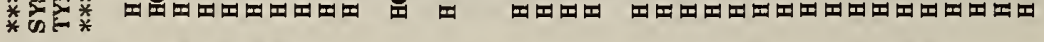

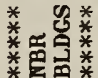

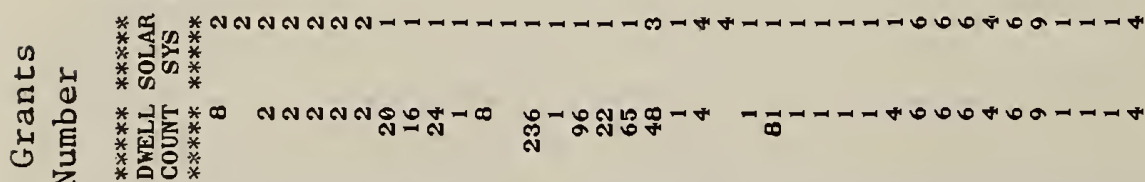

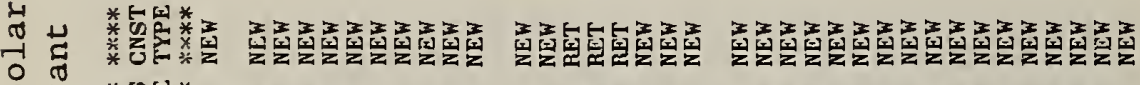

क

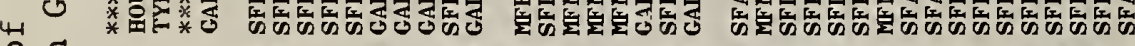
대 ${ }^{*} * \begin{gathered}* \\ *\end{gathered}$

$\lambda$

के वृ

岁
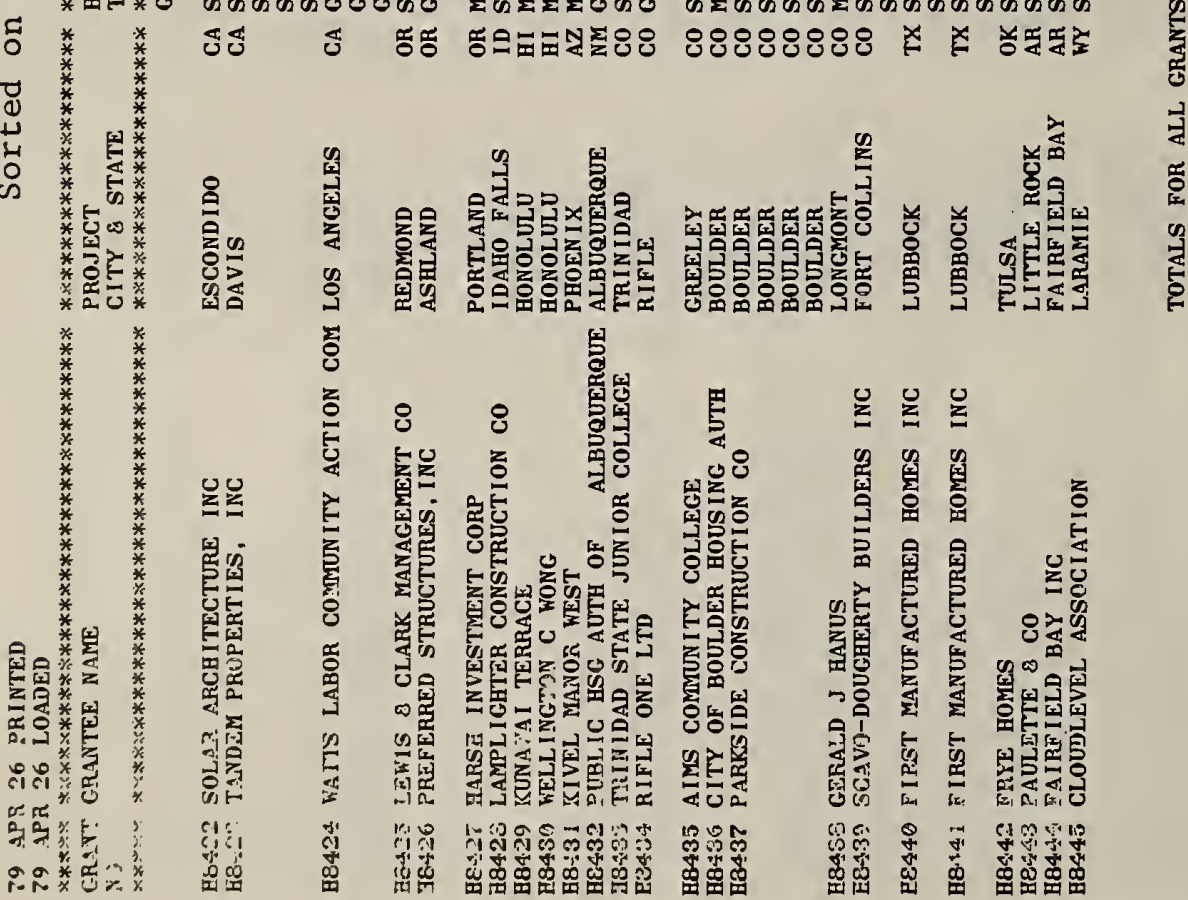
4.3 SUMMARY OF SOLAR GRANTS SORTED ON MANUFACTURER

WITH COST PER MBTU 


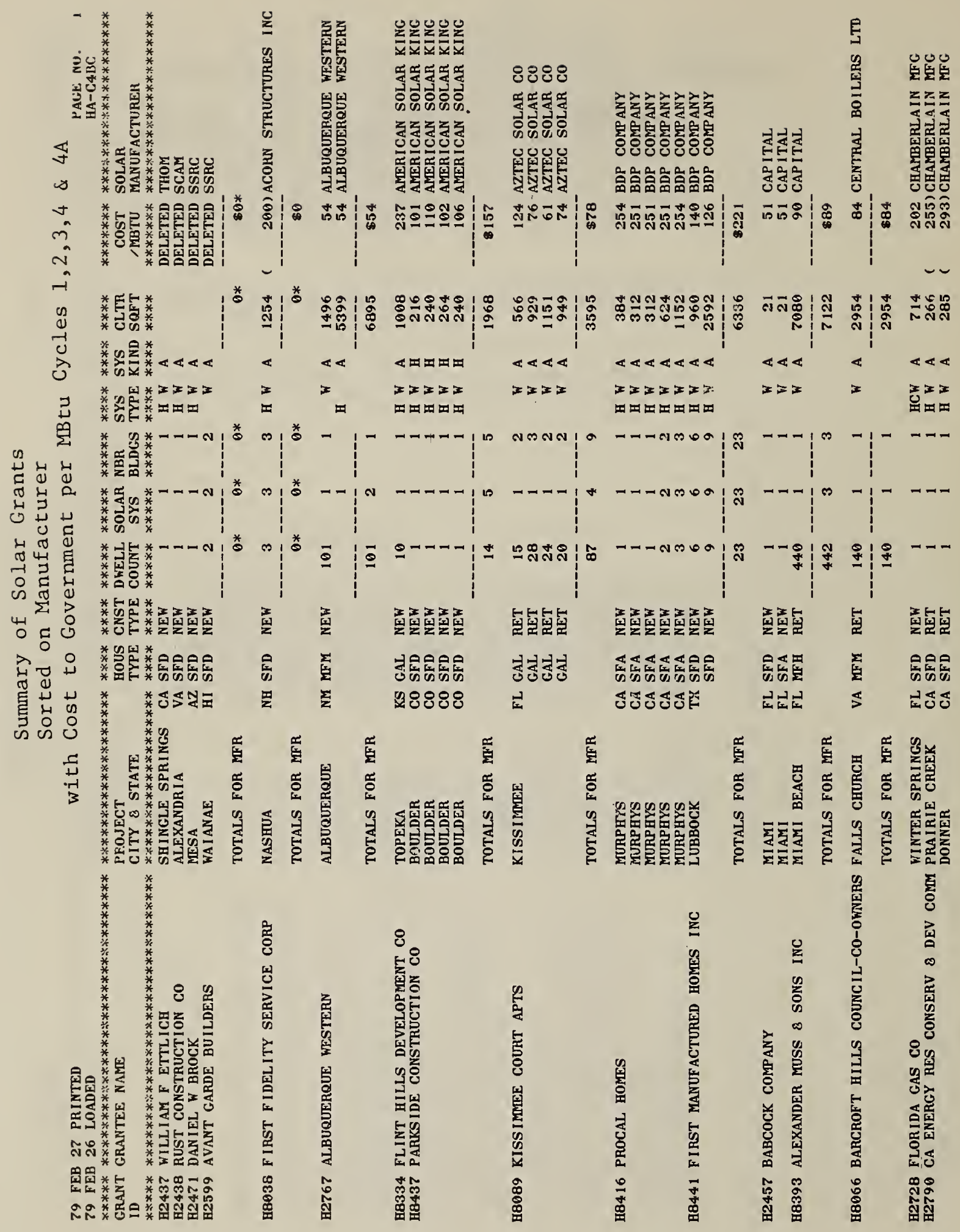




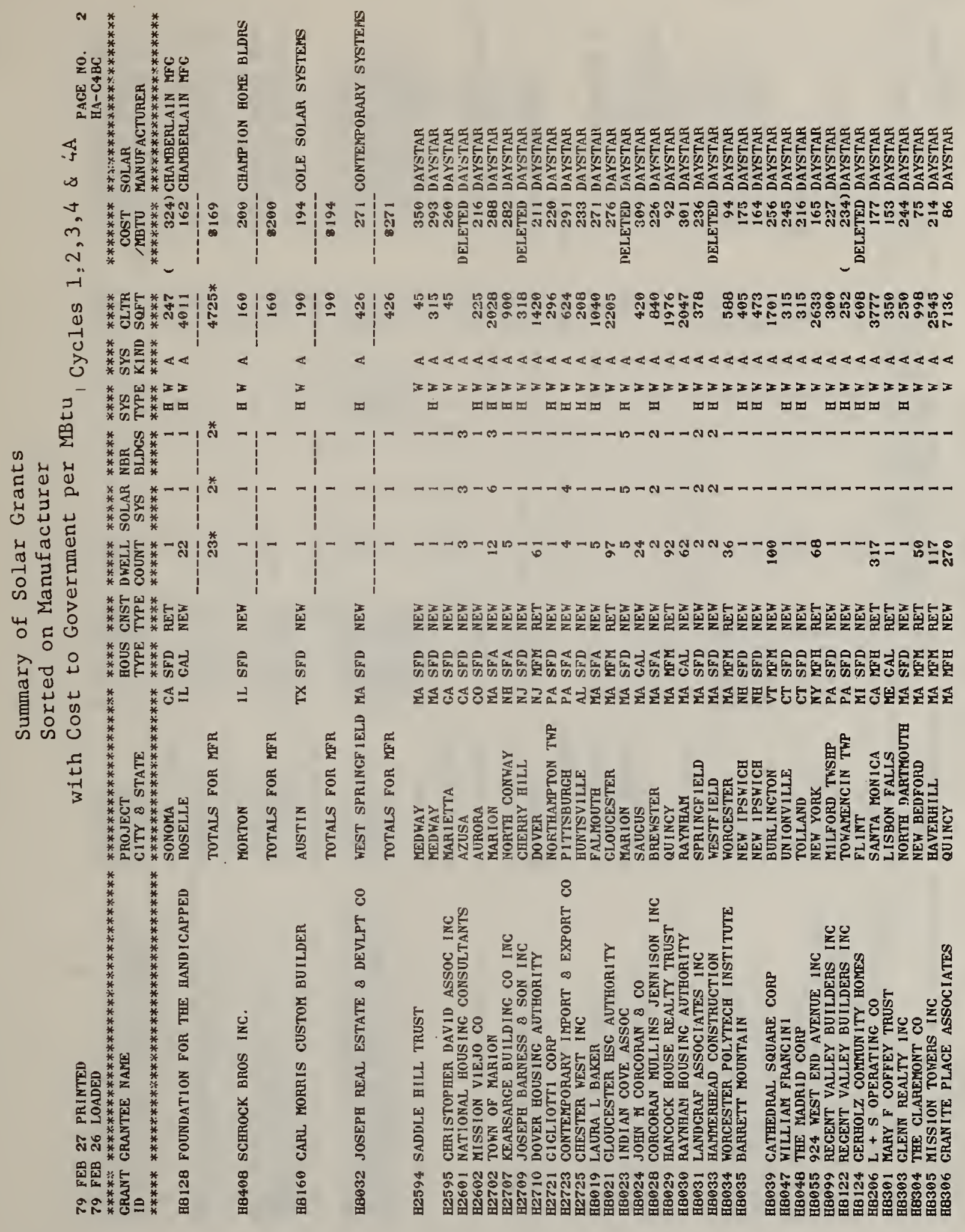




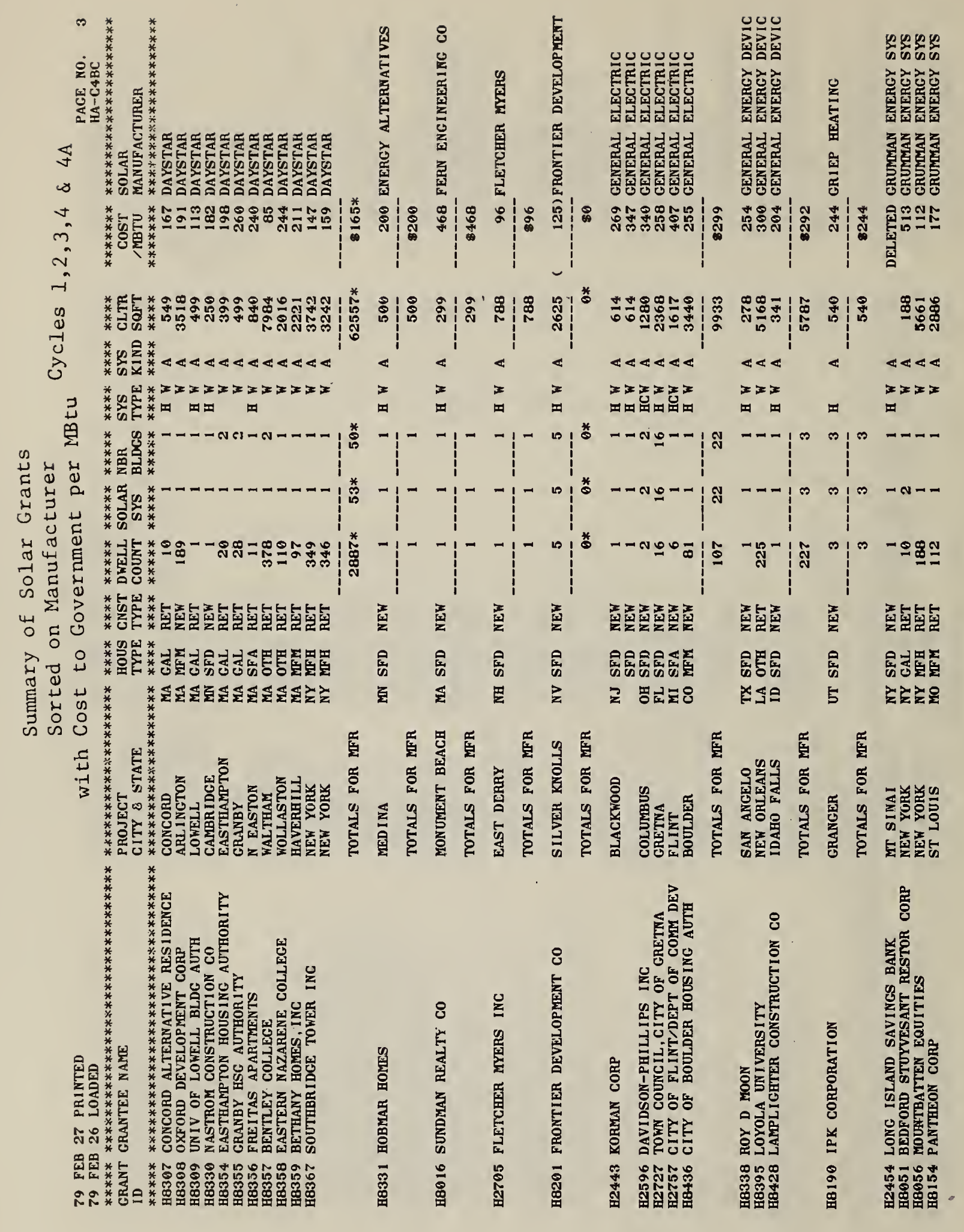




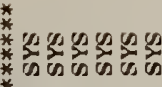

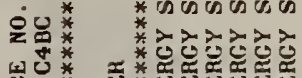

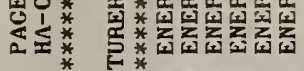

子

む

논

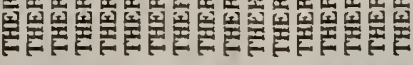

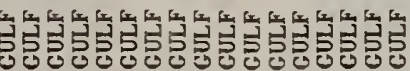

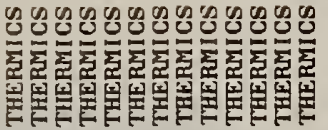
으응ㅇㅇㅇㅇㅇㅇㅇㅇㅇㅇㅇㅇㅇㅇㅡ

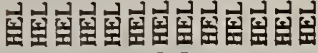

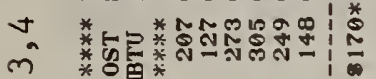

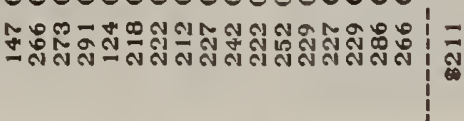

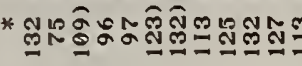

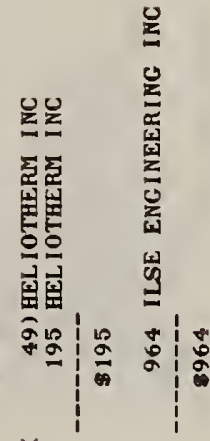

$\rightarrow$

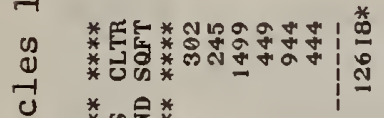

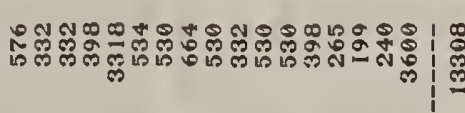

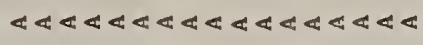

$4<4<4<4<4<4<4$

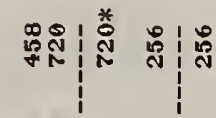

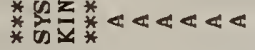

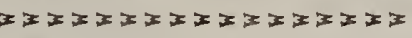

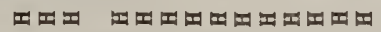

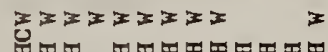

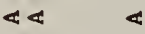

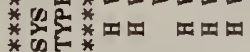

os

急

* $y * \cdots \cdots \cdots-1$

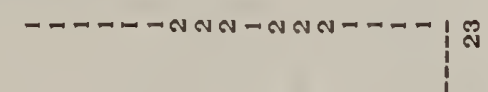

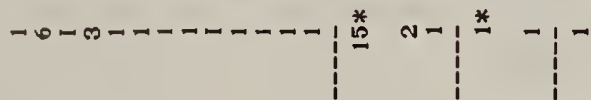

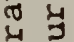

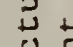

4 过

崩岩

i

$\sum^{\pi}$

㟧

त्रें 우

त्र बु

*䓪菑*

*3)

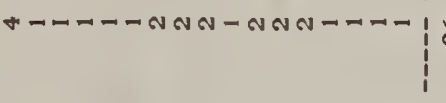

*

等

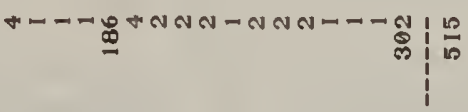

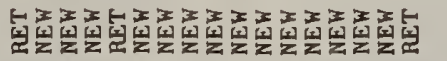

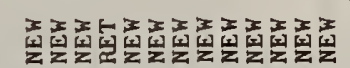

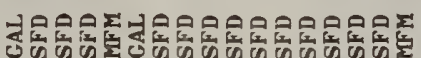

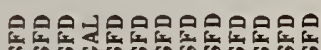

葛

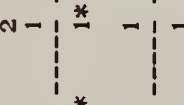

鼻

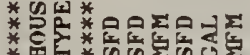

고애

త઼ర

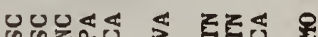

葛 $\mathrm{N}$

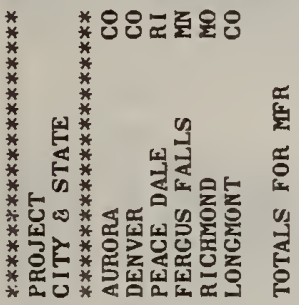

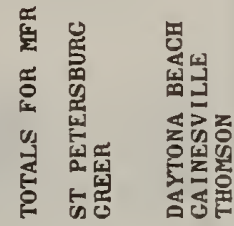

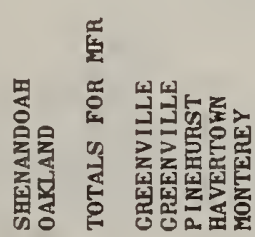

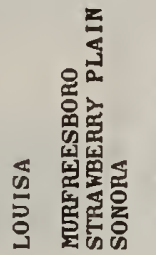

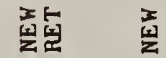

㩆总

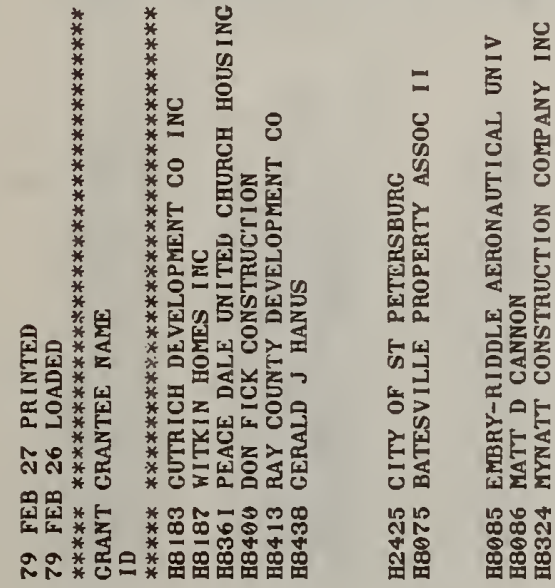

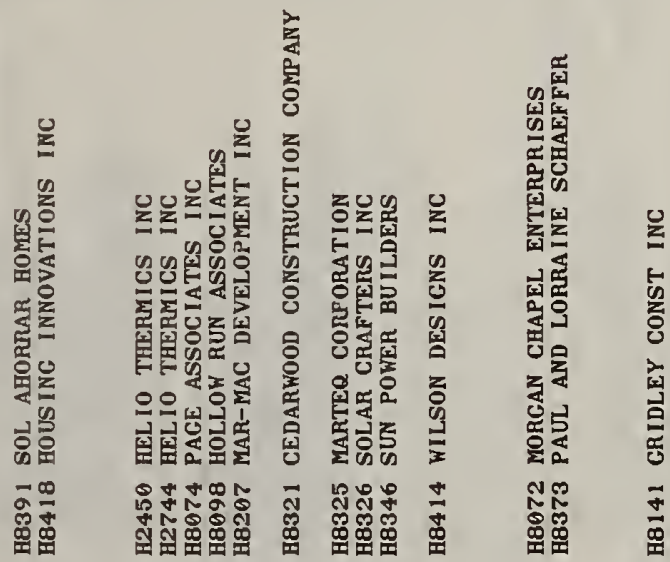




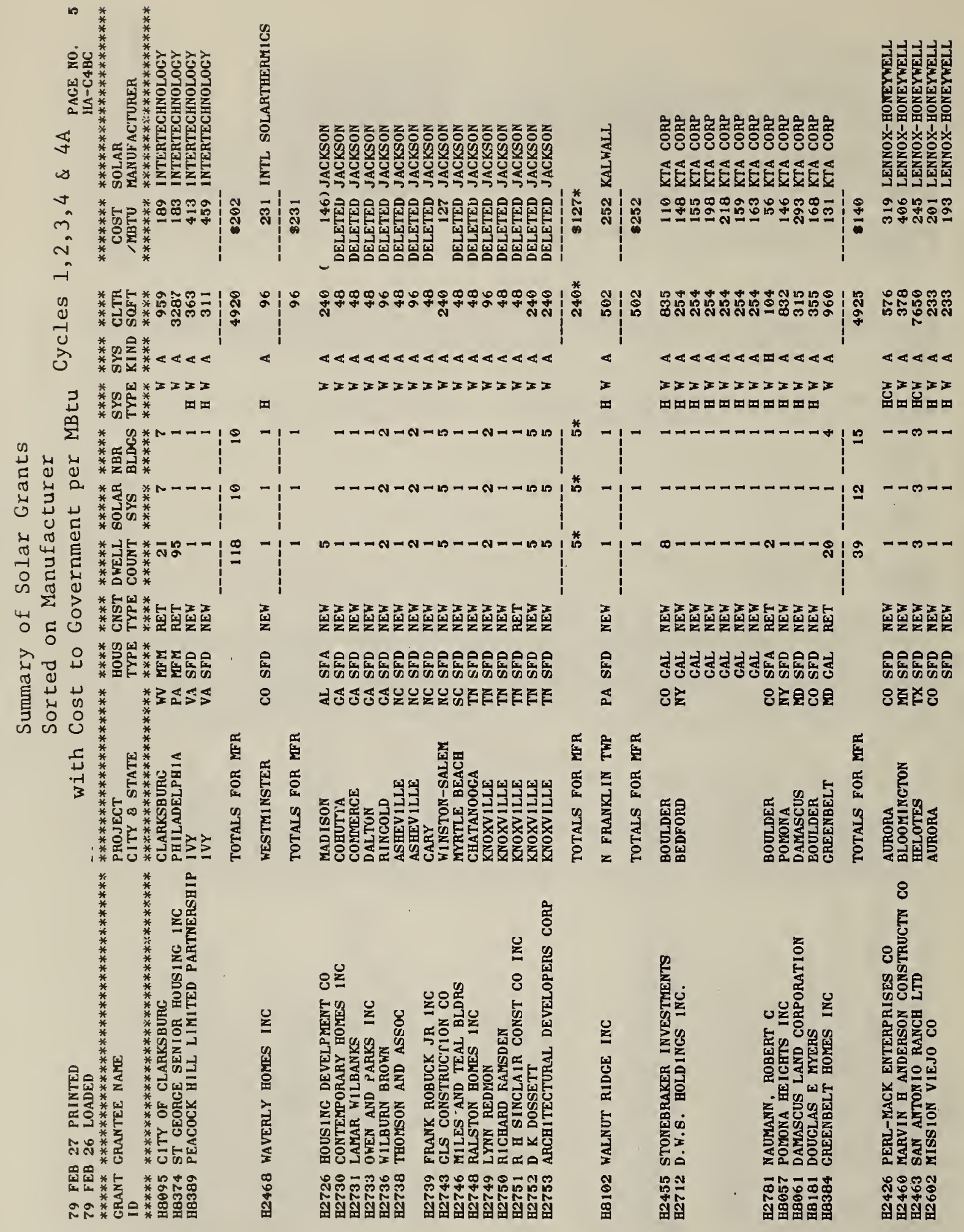




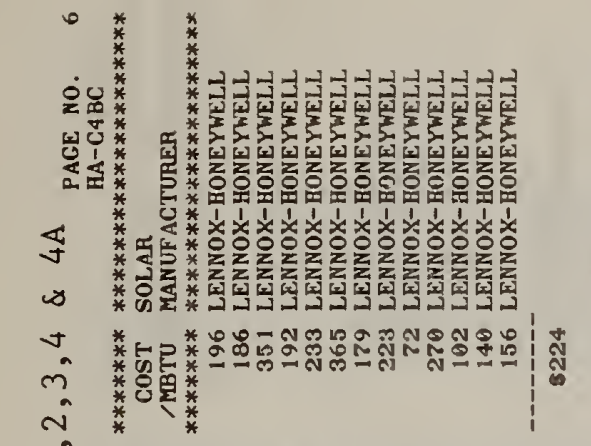

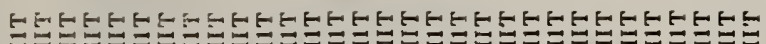

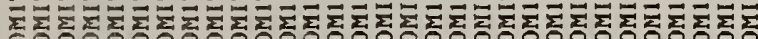

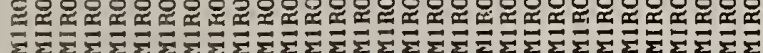

跑|

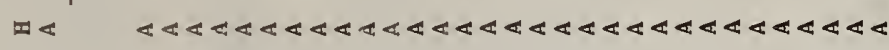

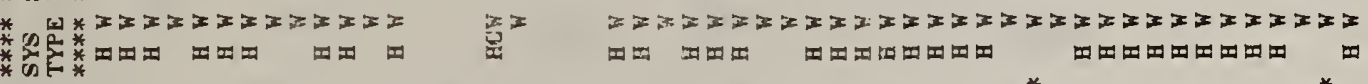

党

*

N- $\mid$ |

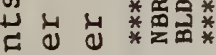

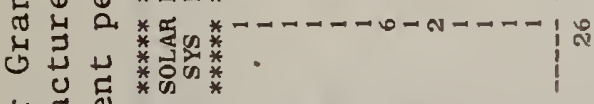

4

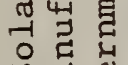

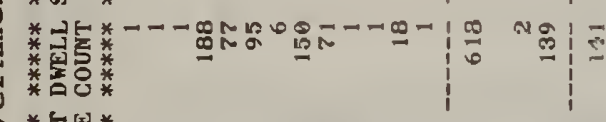

$\left.N \rightarrow\right|^{\infty}$

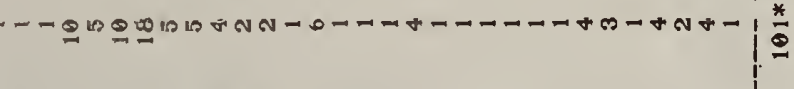

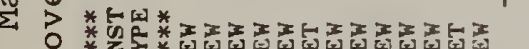

出

용

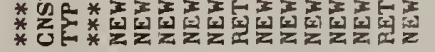

琶魚

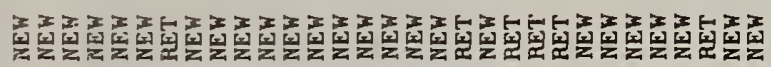

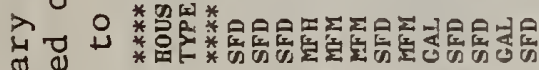

是罢

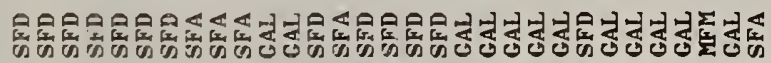

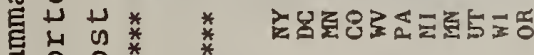

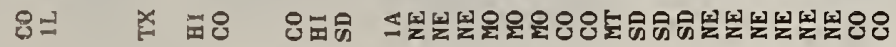

का i 0
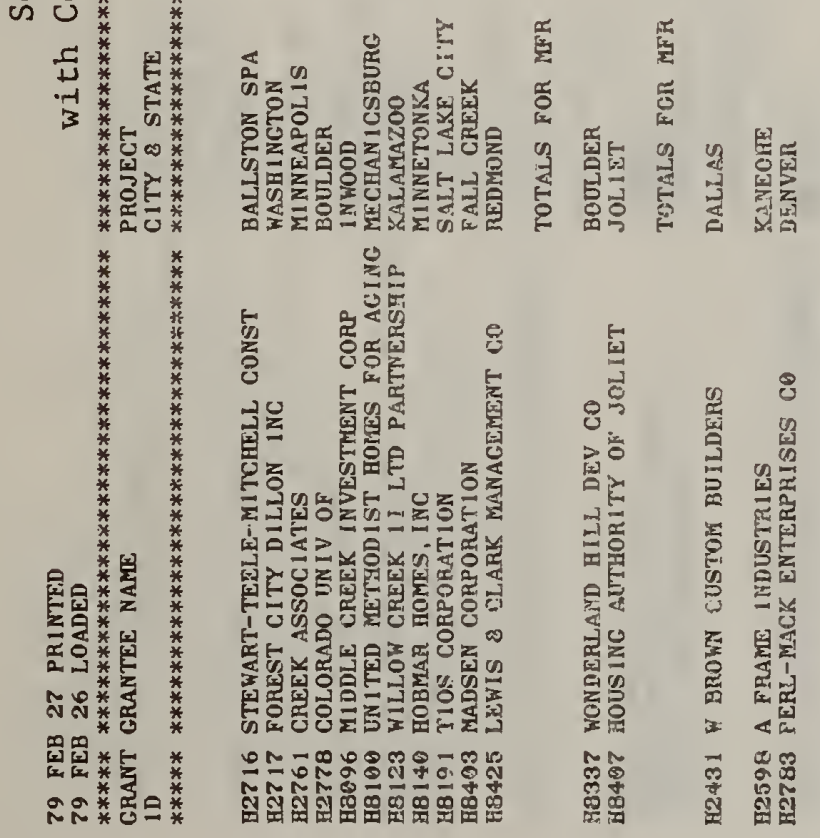

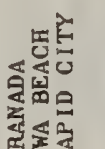

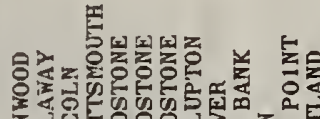

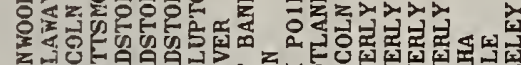

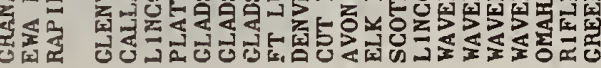

窗

庵

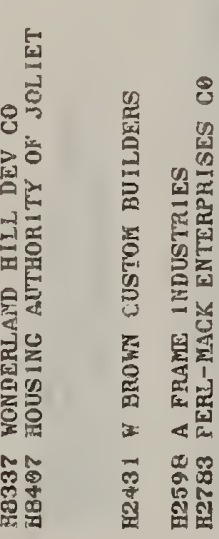

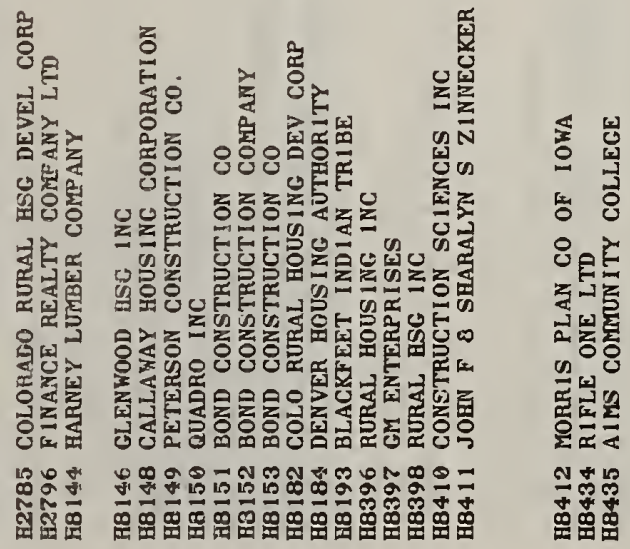




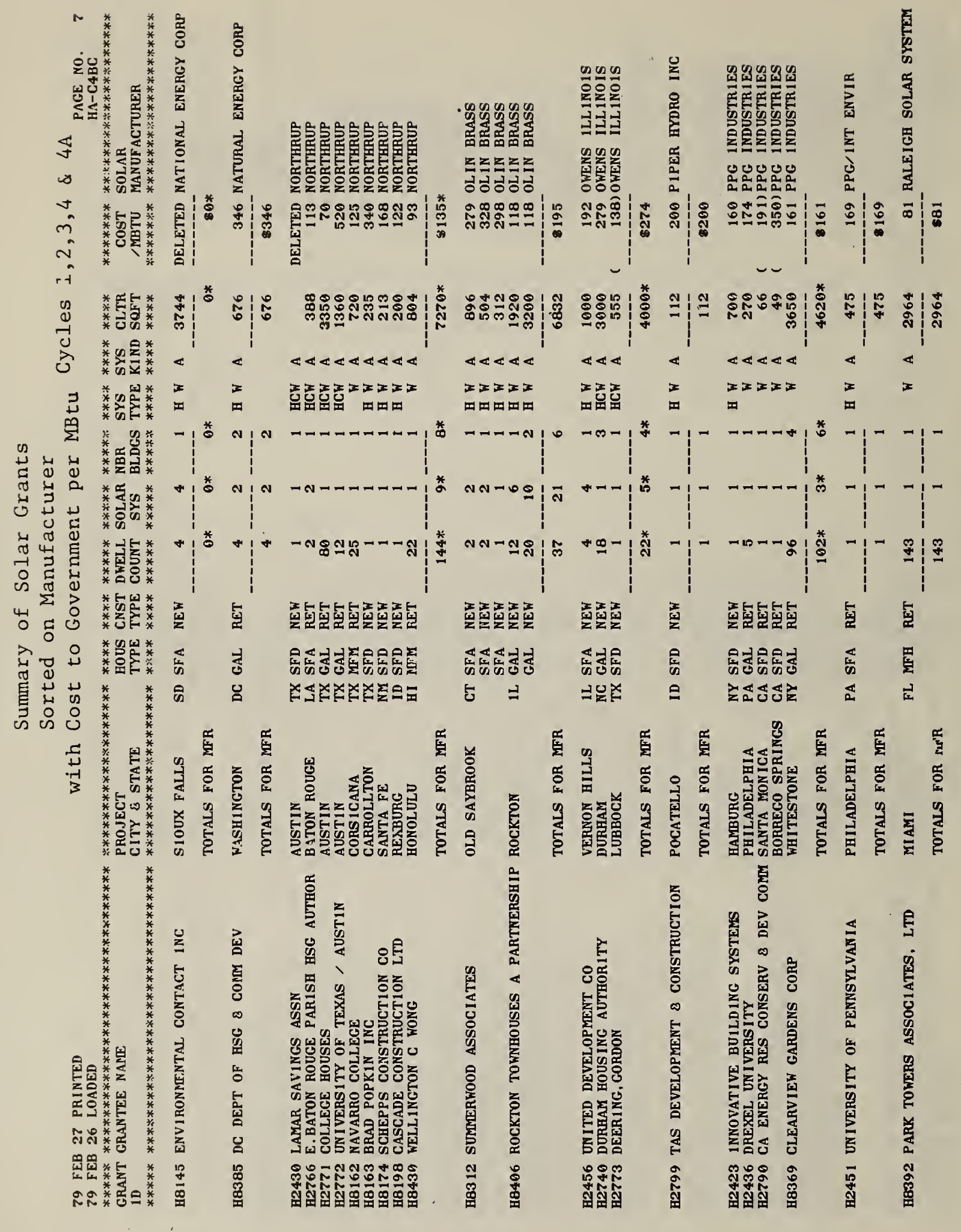



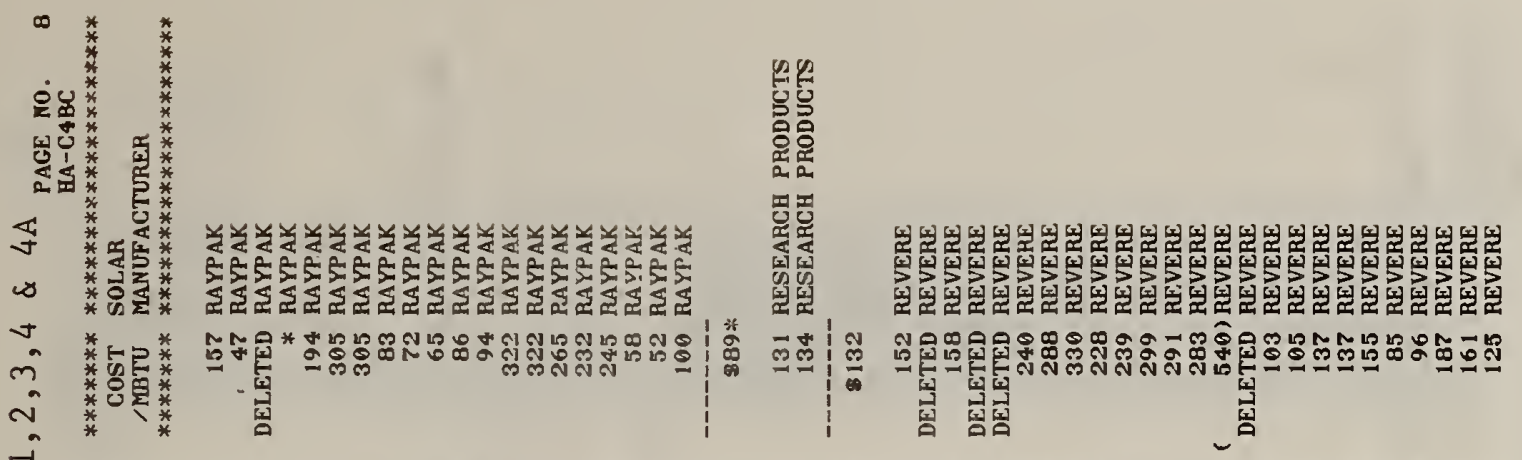

踝昌

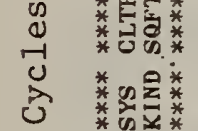
(4)

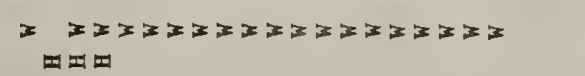

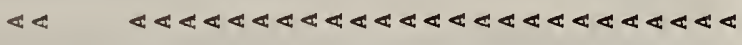

$\geq$

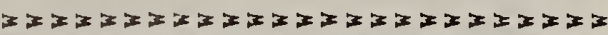

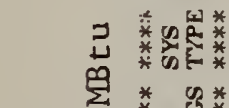

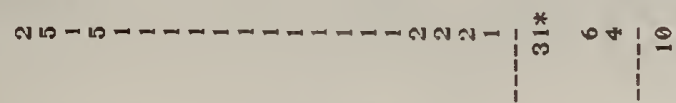

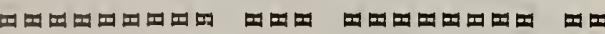

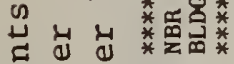

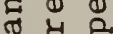

* ⿷匚

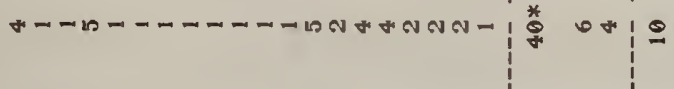

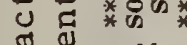

मี

*

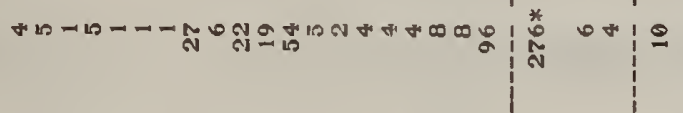

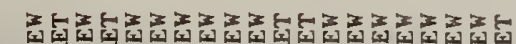

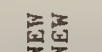

势

㟧

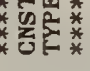

它

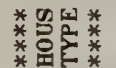

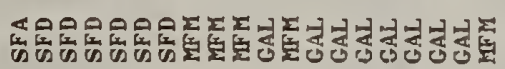

昆昆

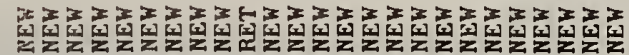

芩

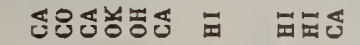

两

葆

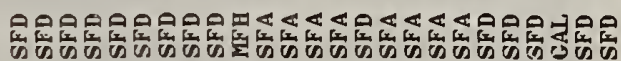

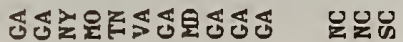

퐁폴토엉ㅇ

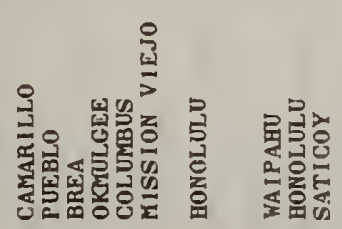

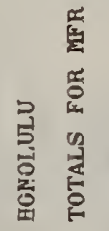

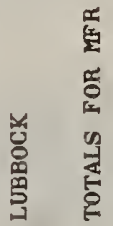

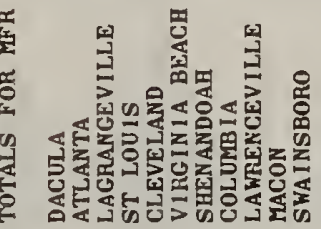

㦈兽

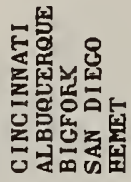

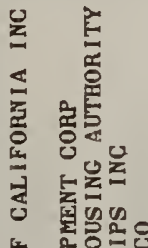

E。

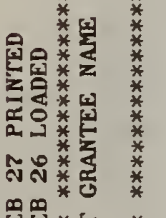

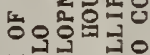

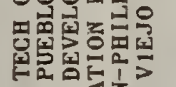

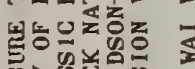

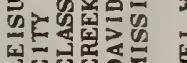

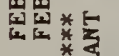

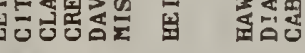

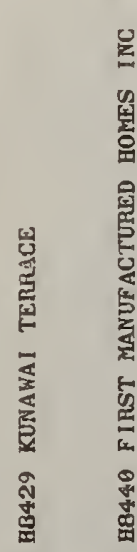
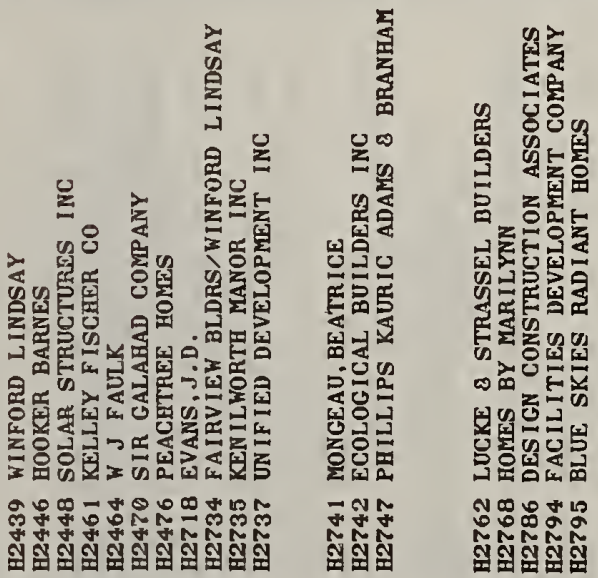


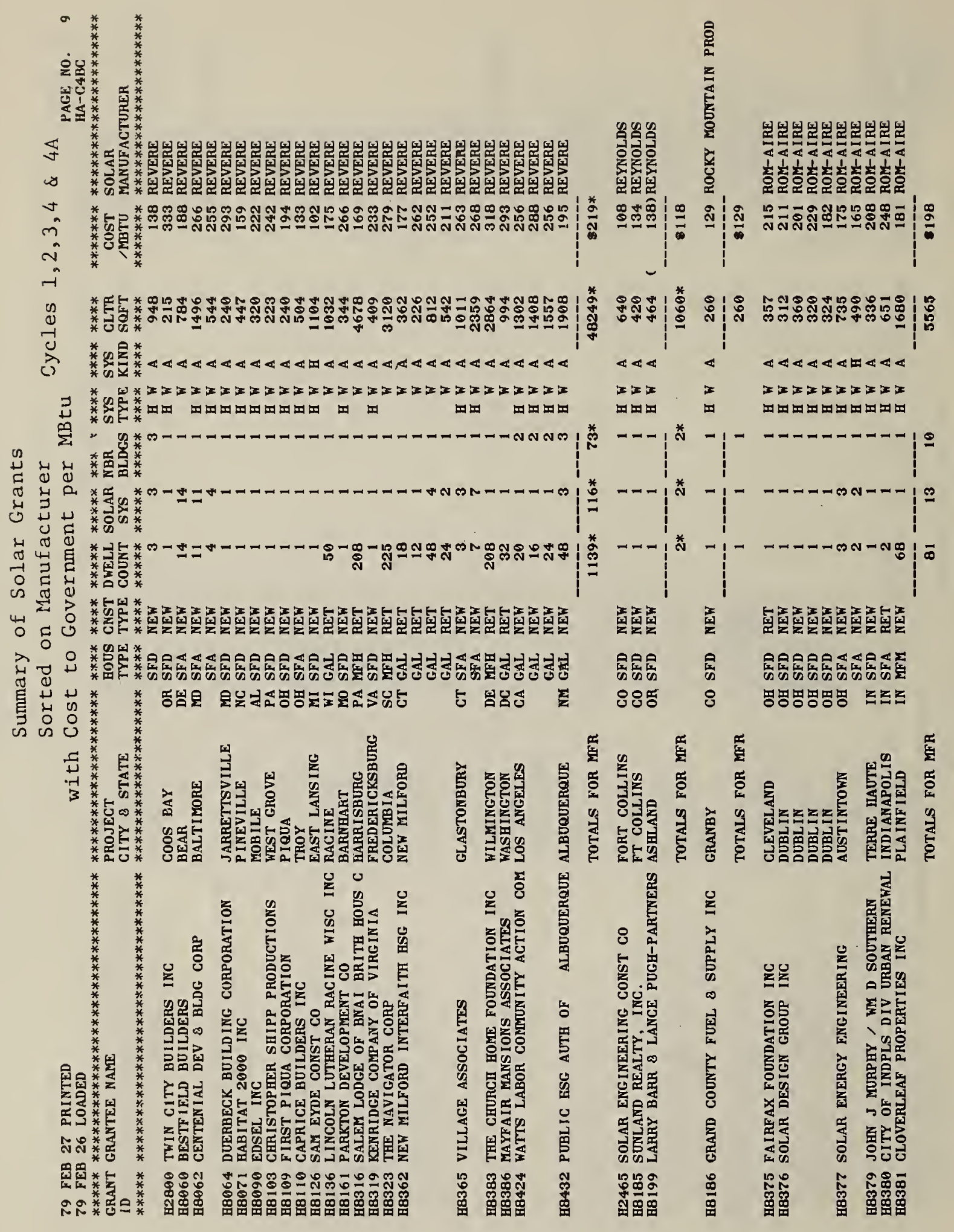




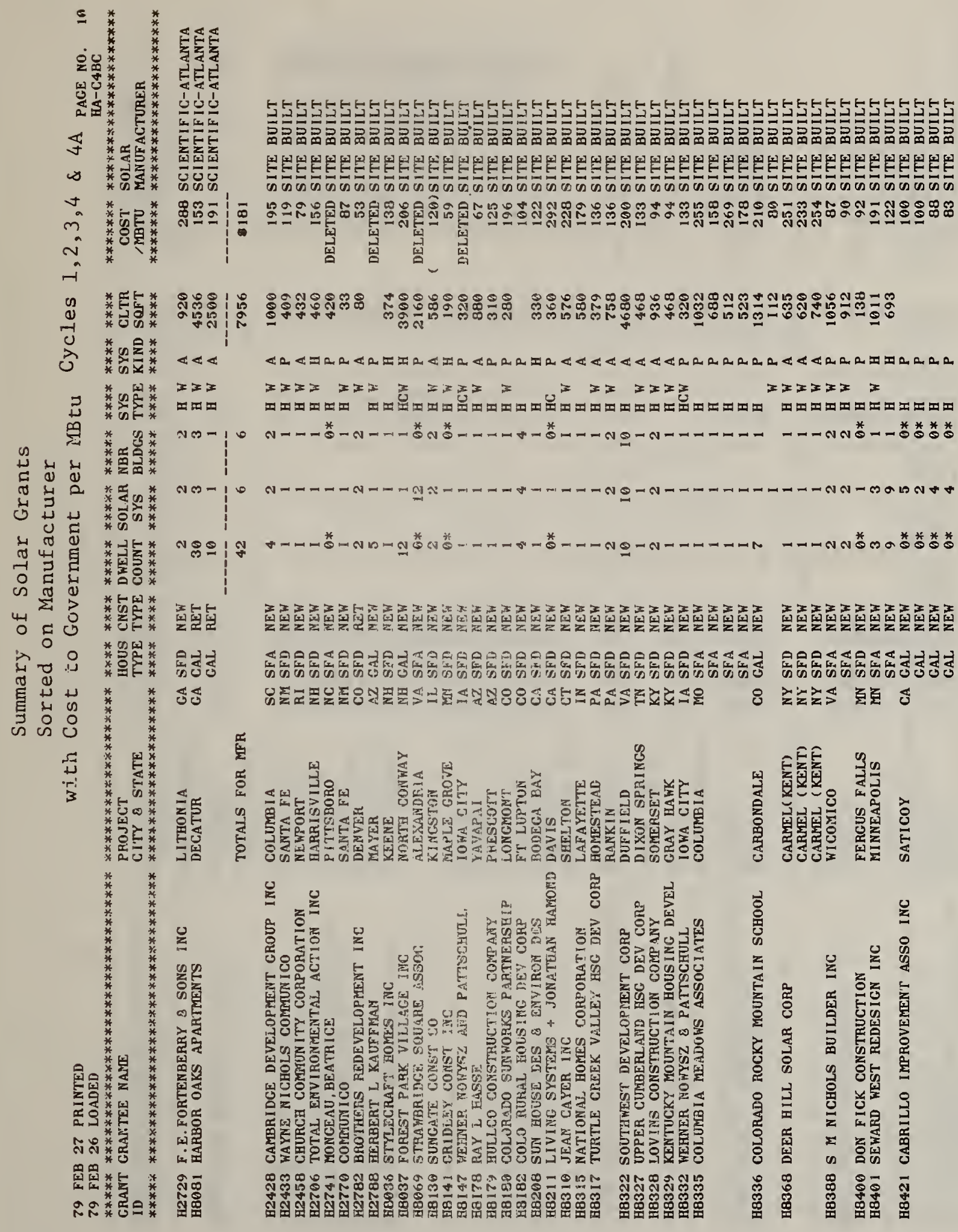




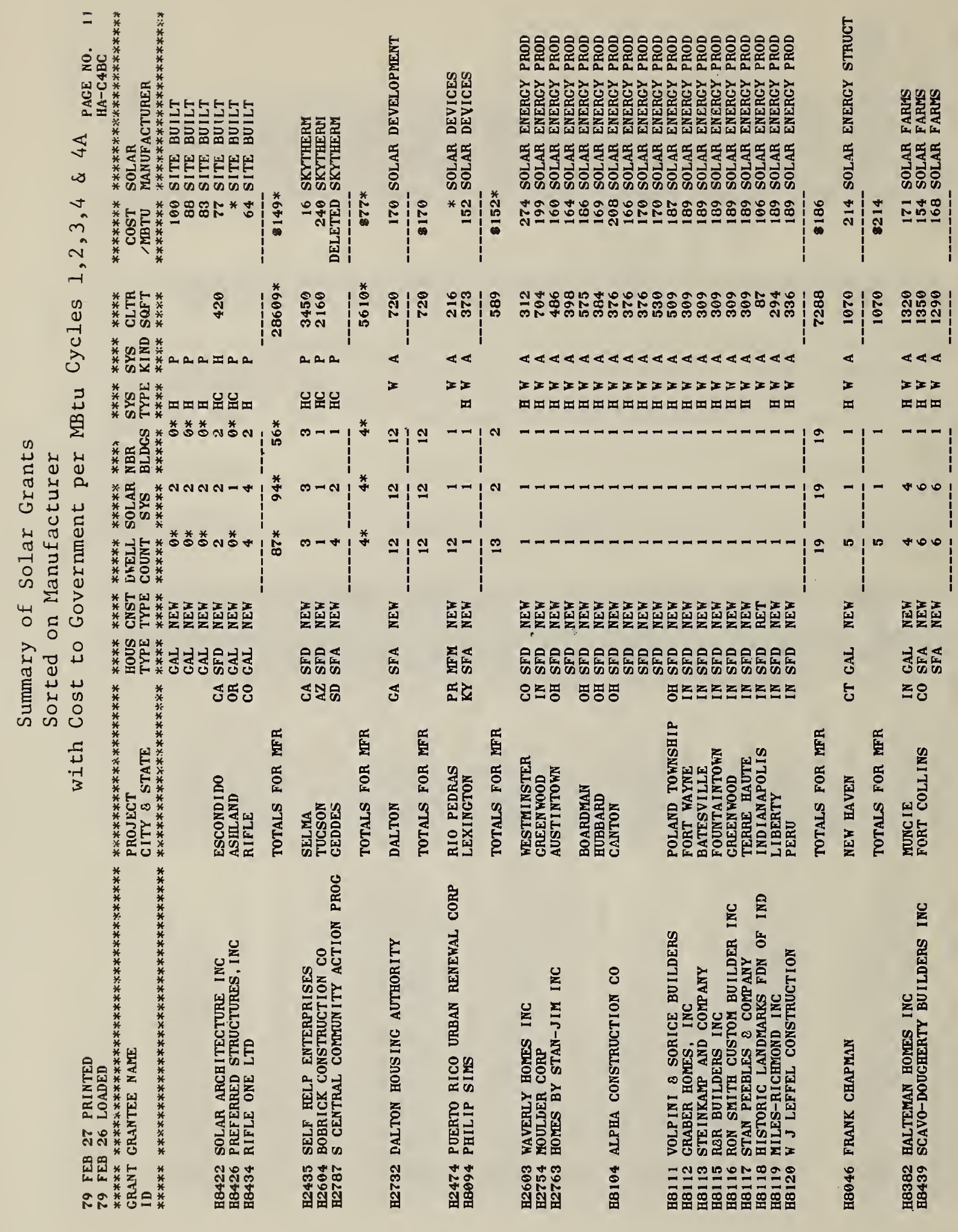




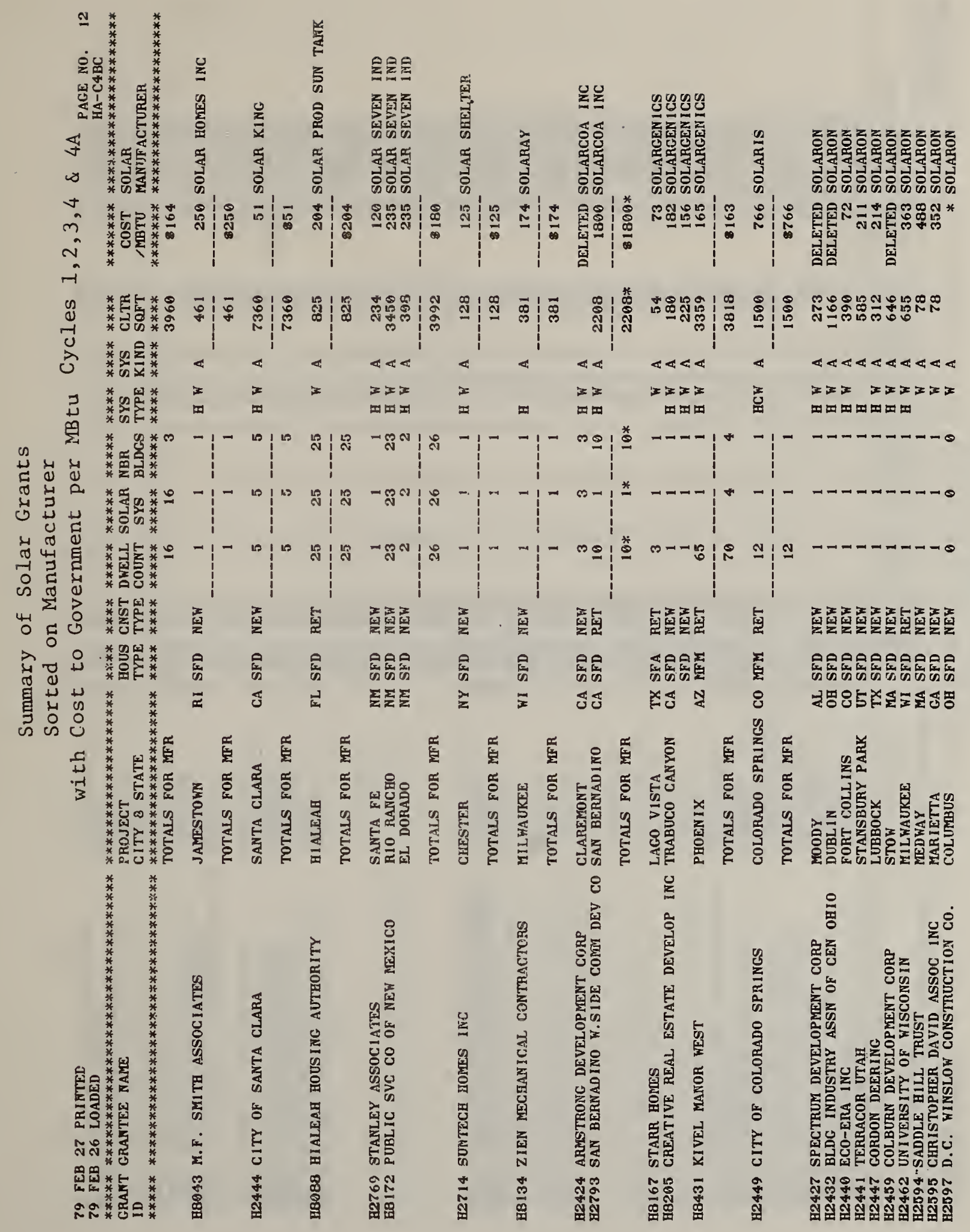




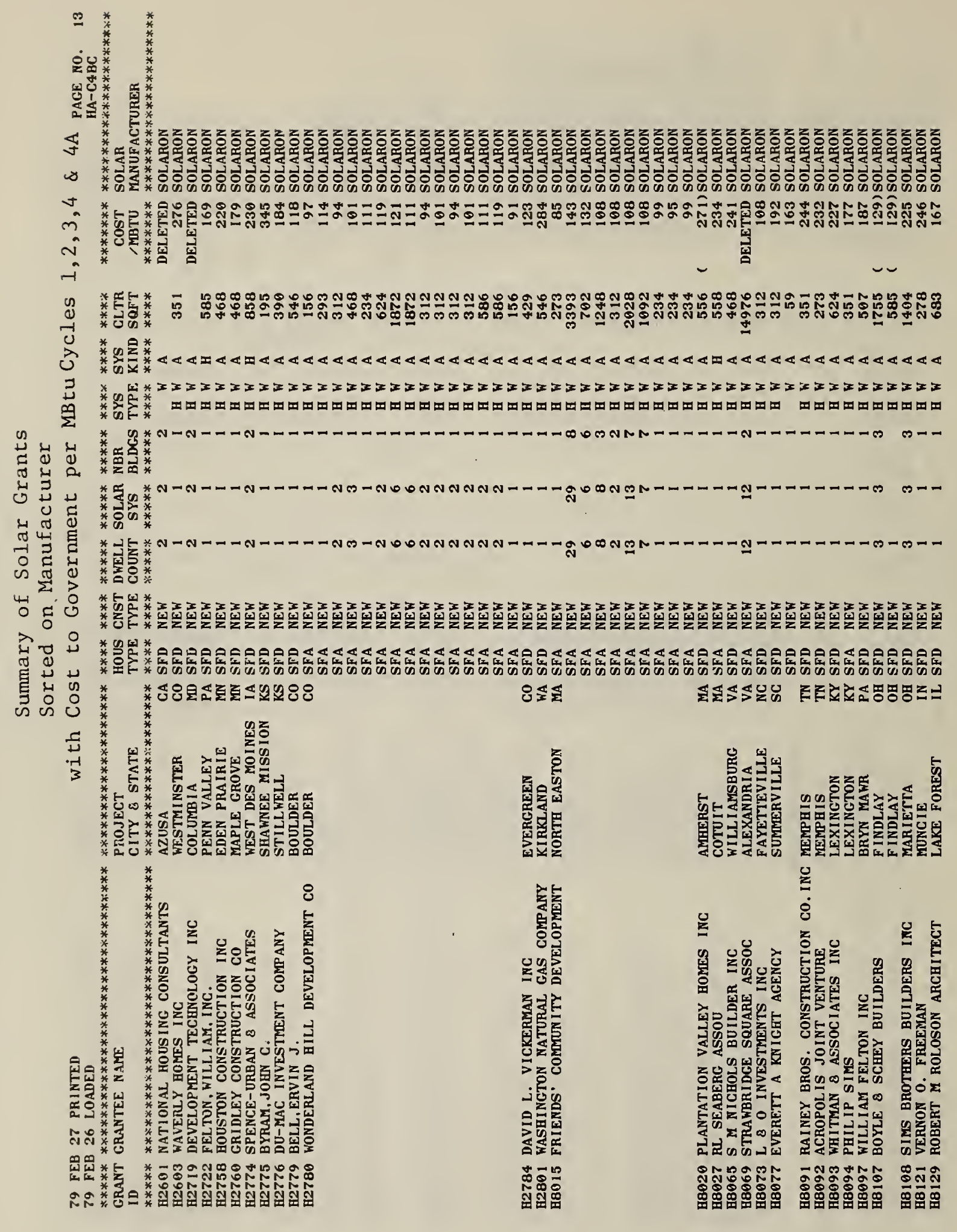




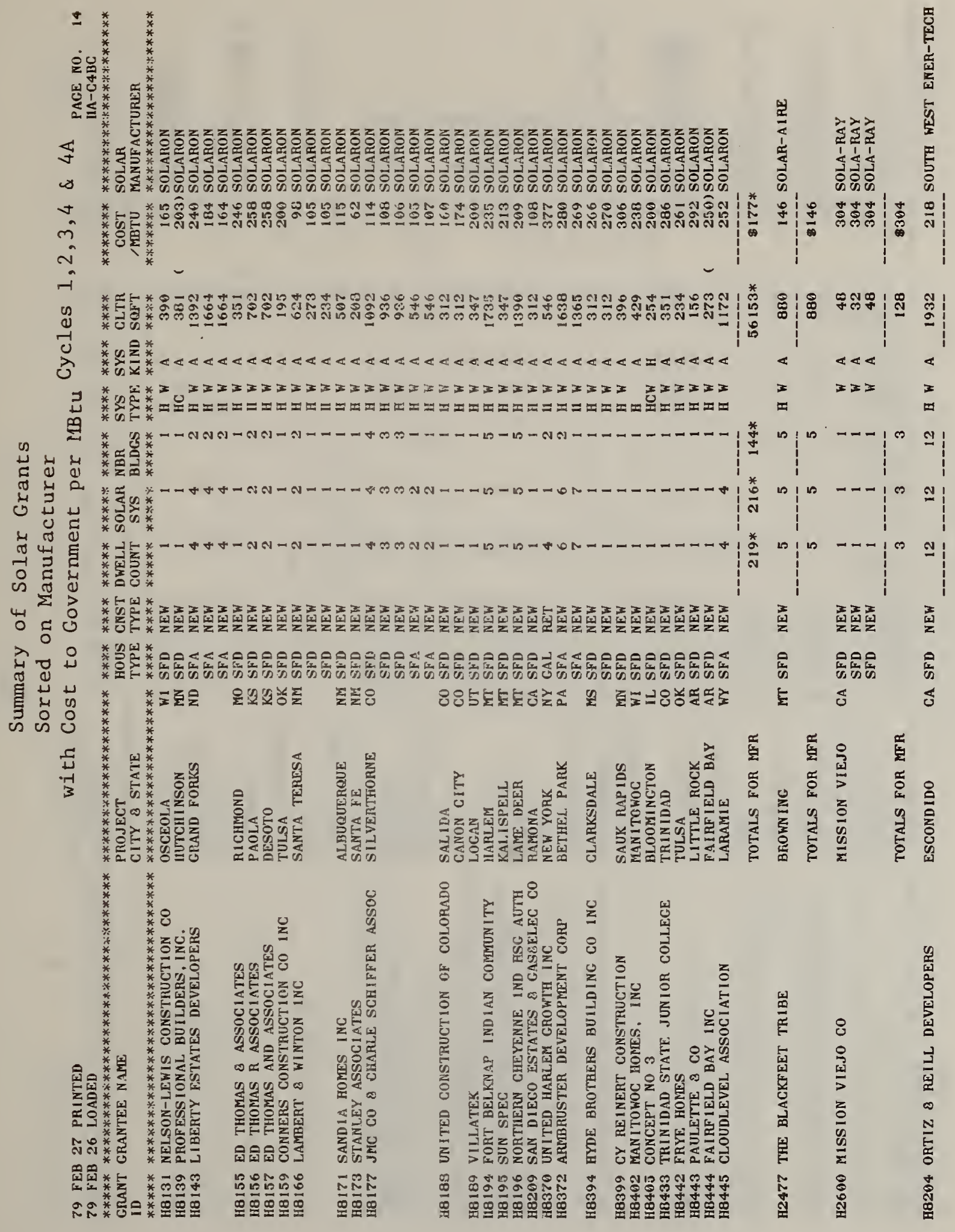




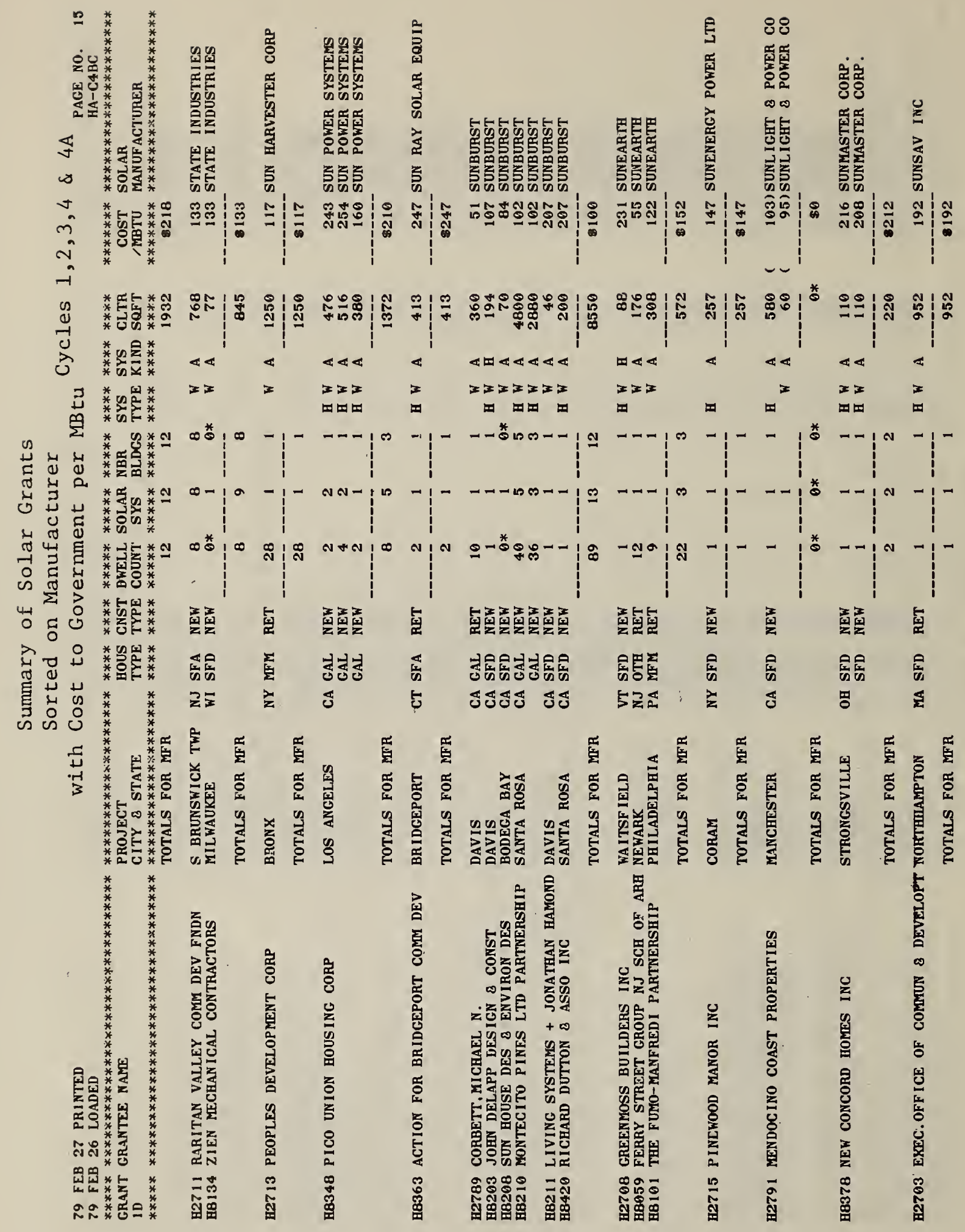




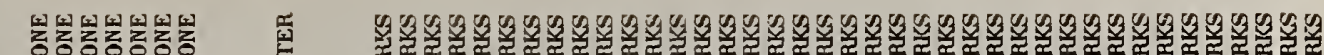

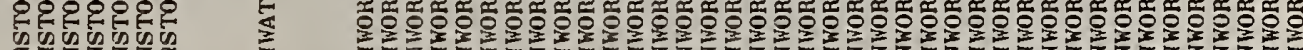

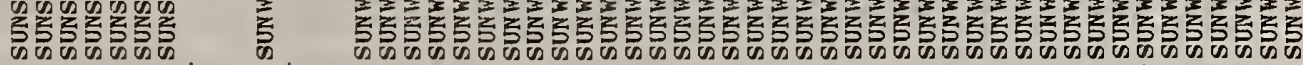

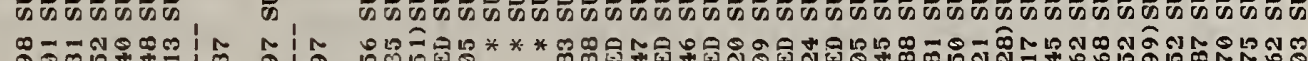

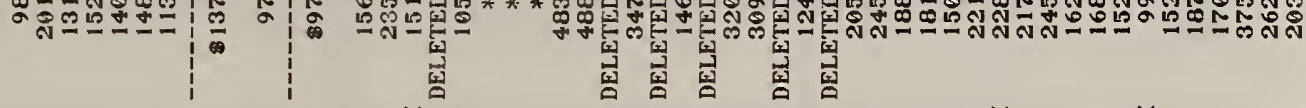

n

ثै

ช

斗

U

幽苾

길

is

世

岁䓃

द्षे

कृष

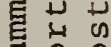

की

点

*

손준

중

ลิำ

䛼䛼䓍

国思菜势

* น *

* 동 *

*ax

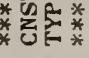

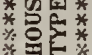

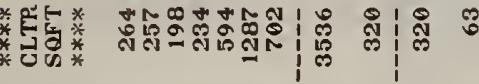

经会*

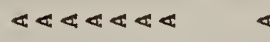

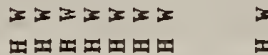

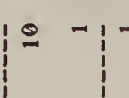

* 吃莫

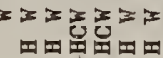

四

回四理

田

田

四田田四四四 **<smiles>CCO[V](I)(I)I(C)I</smiles>

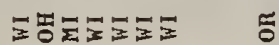

\section{西}

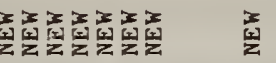

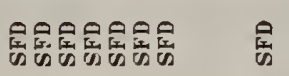

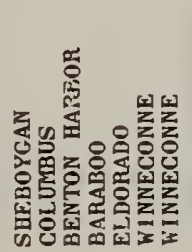

\section{.}

놇용

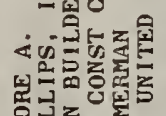

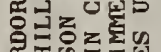
氙以车 舟我的

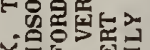

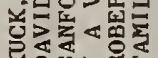
ㄴํํำำตัต

흥

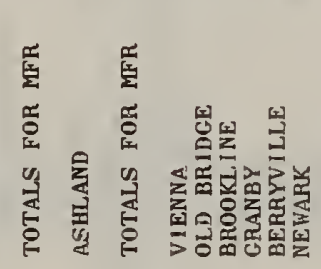

ヘヘ*心口*

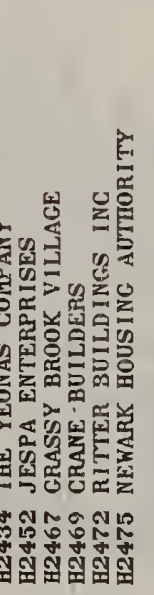

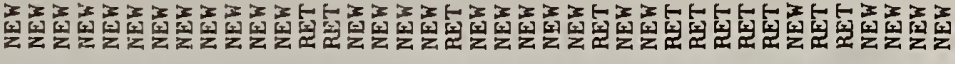

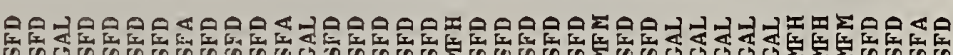

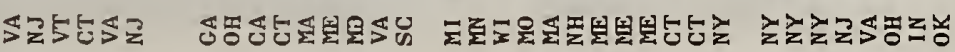

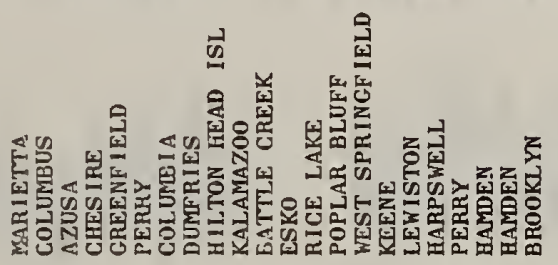

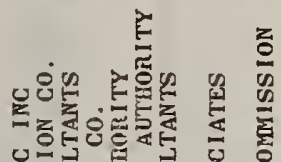

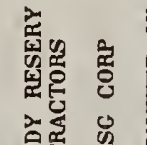
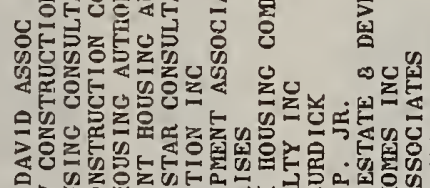

×u

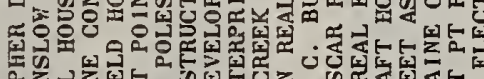

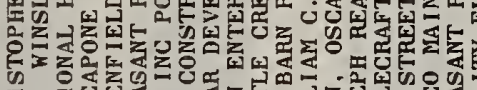

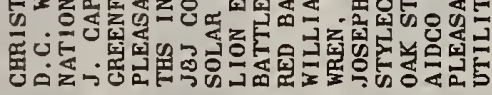

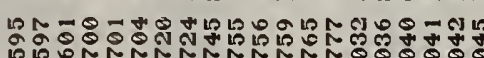

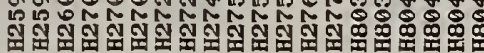
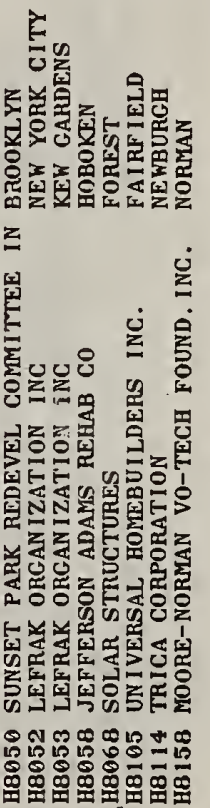


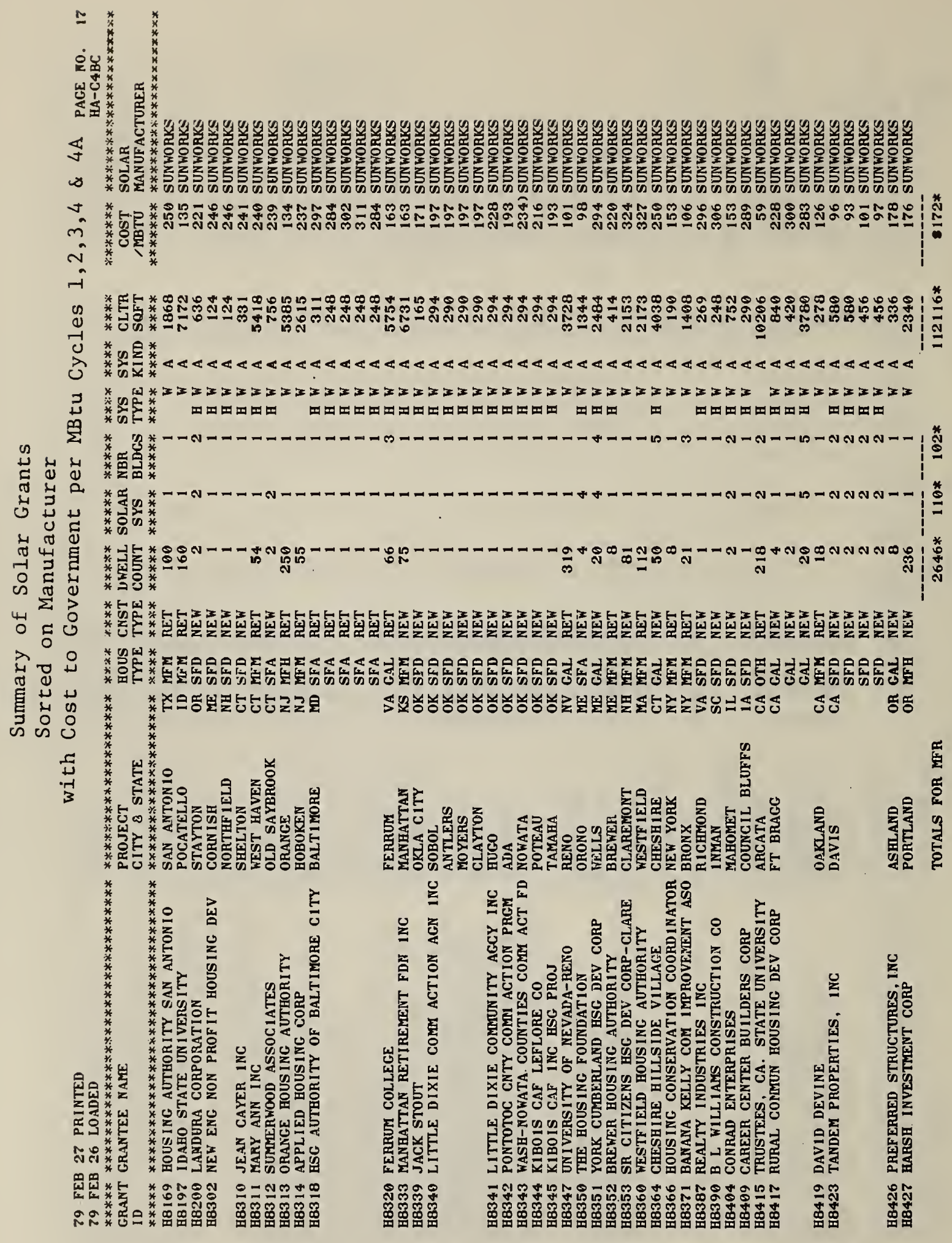



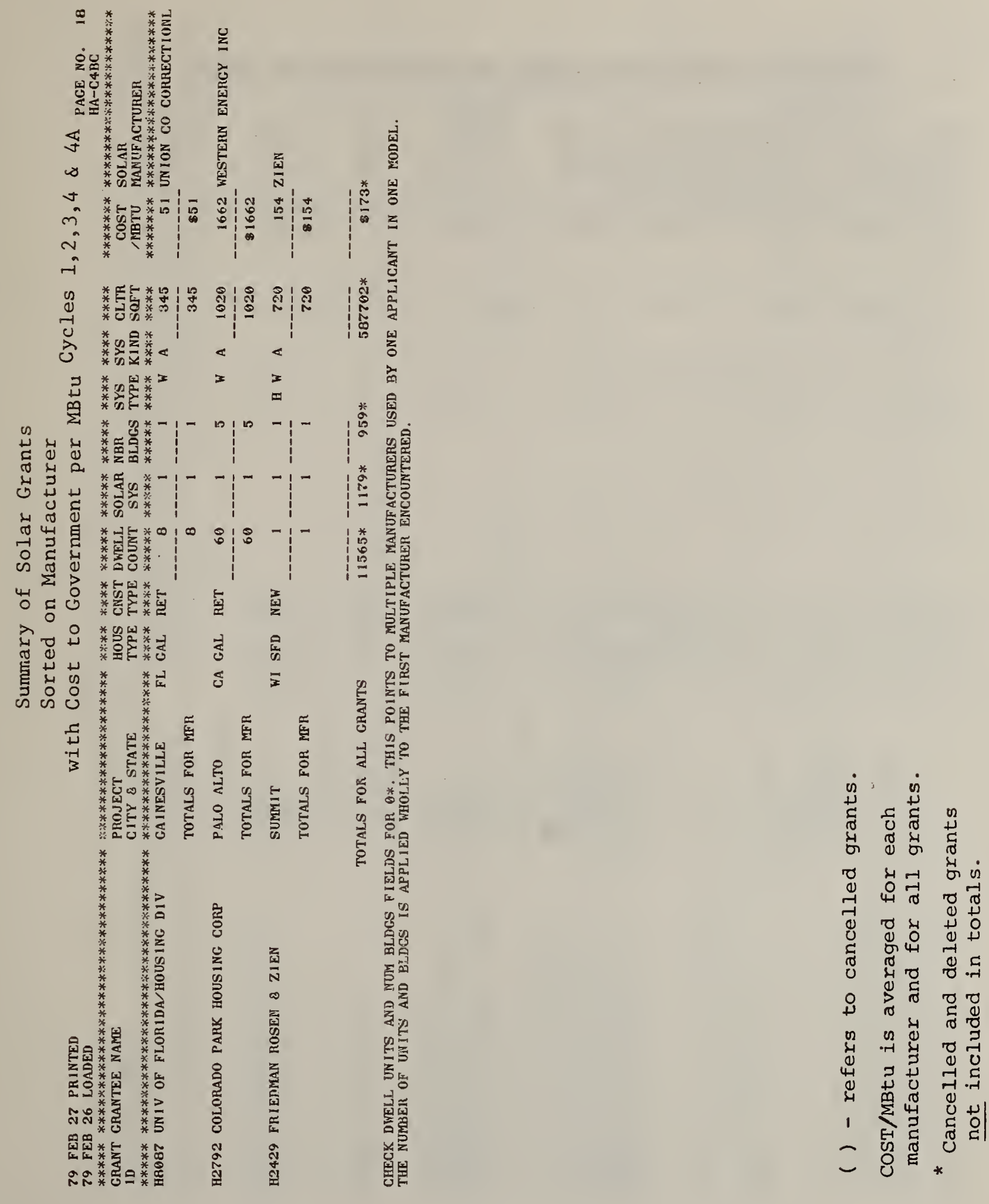
4.4 SUMMARY OF SOLAR GRANTS SORTED ON PROJECT CITY AND STATE 


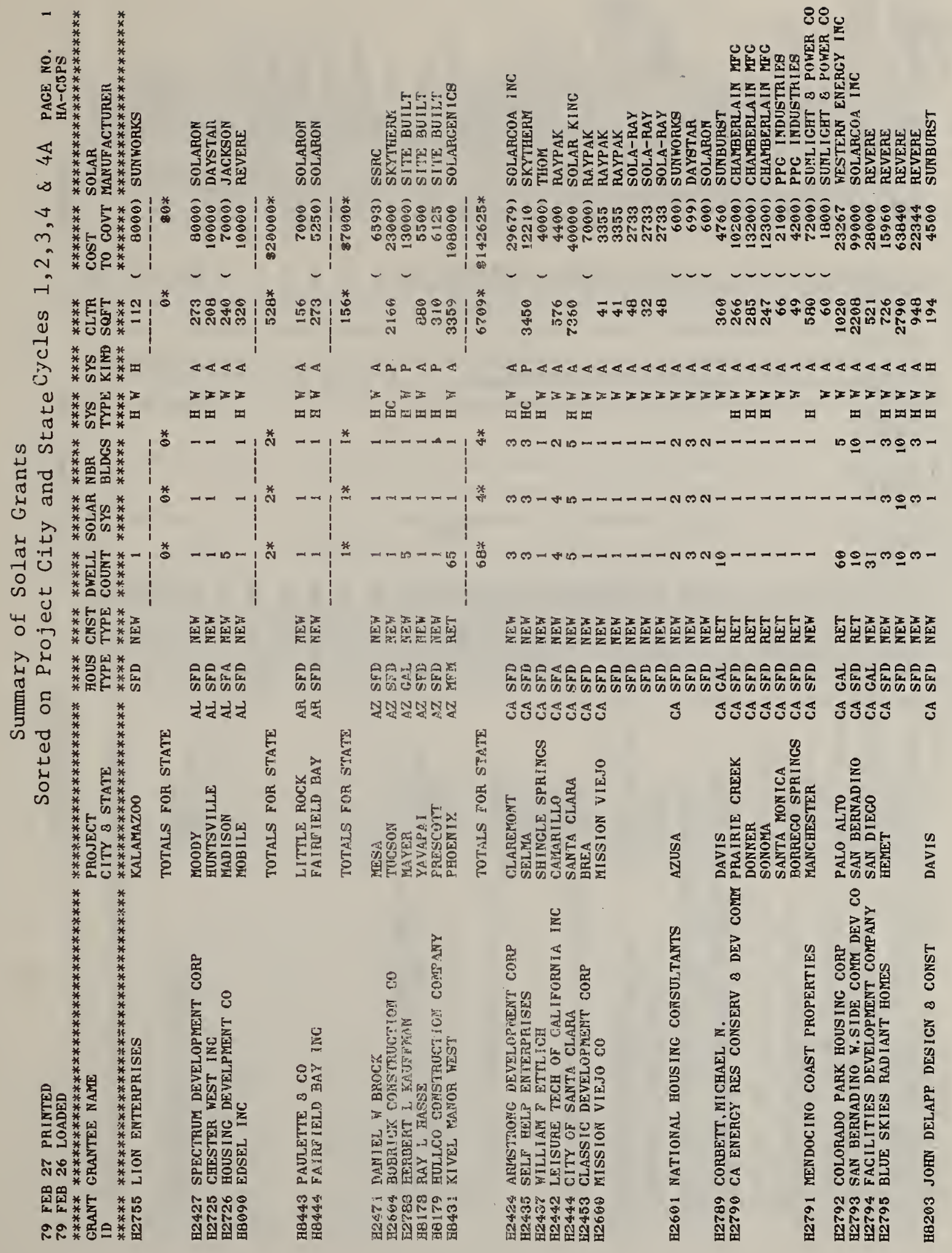




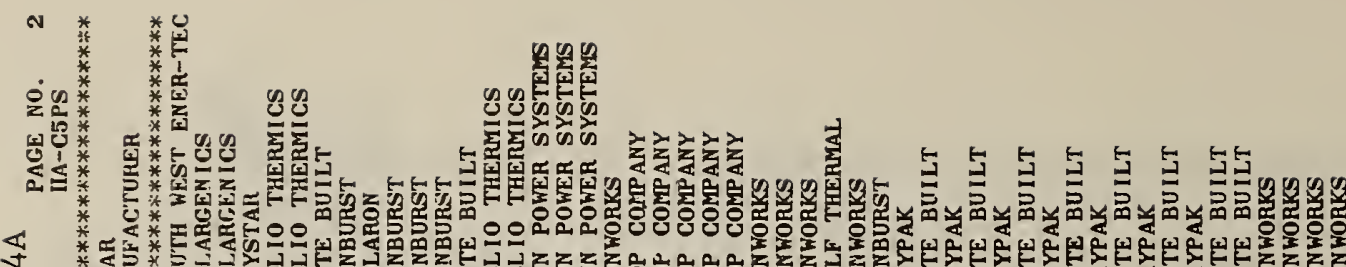

4 苦

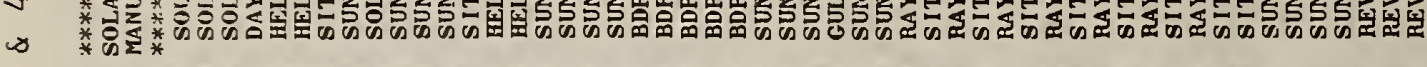

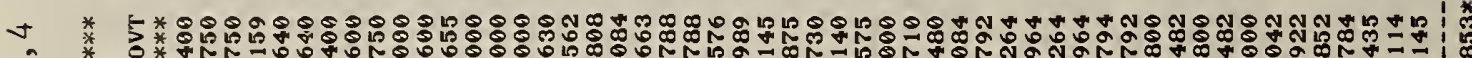

i * * * *

†

Uे

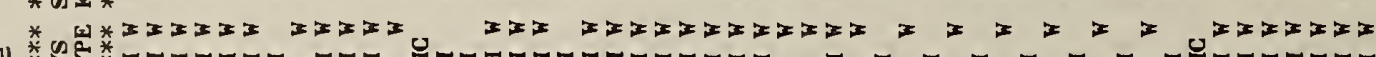

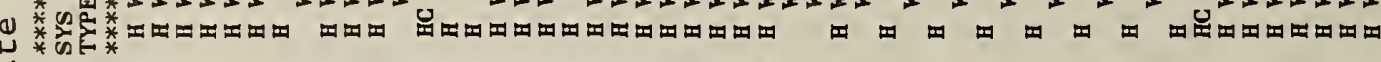

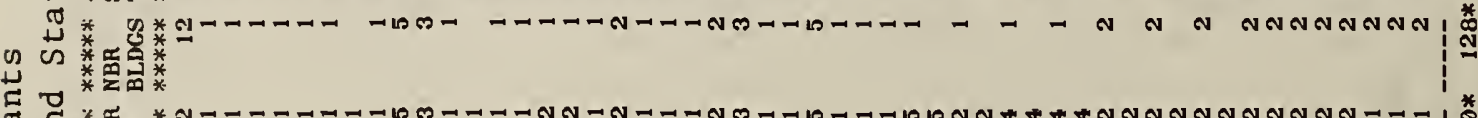

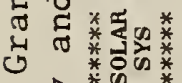

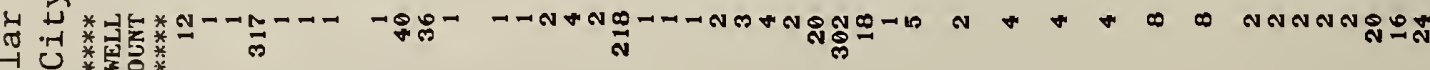
年 U*

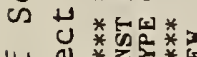

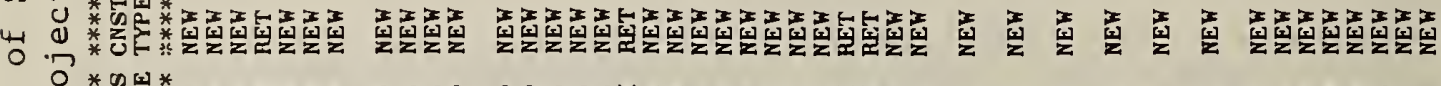

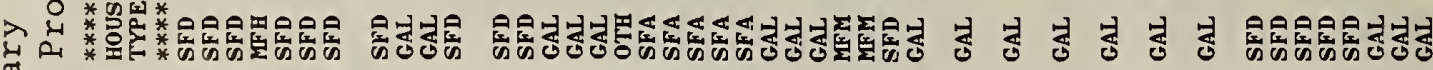

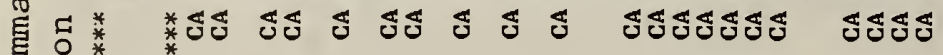

ชิษ ธ્ฮ

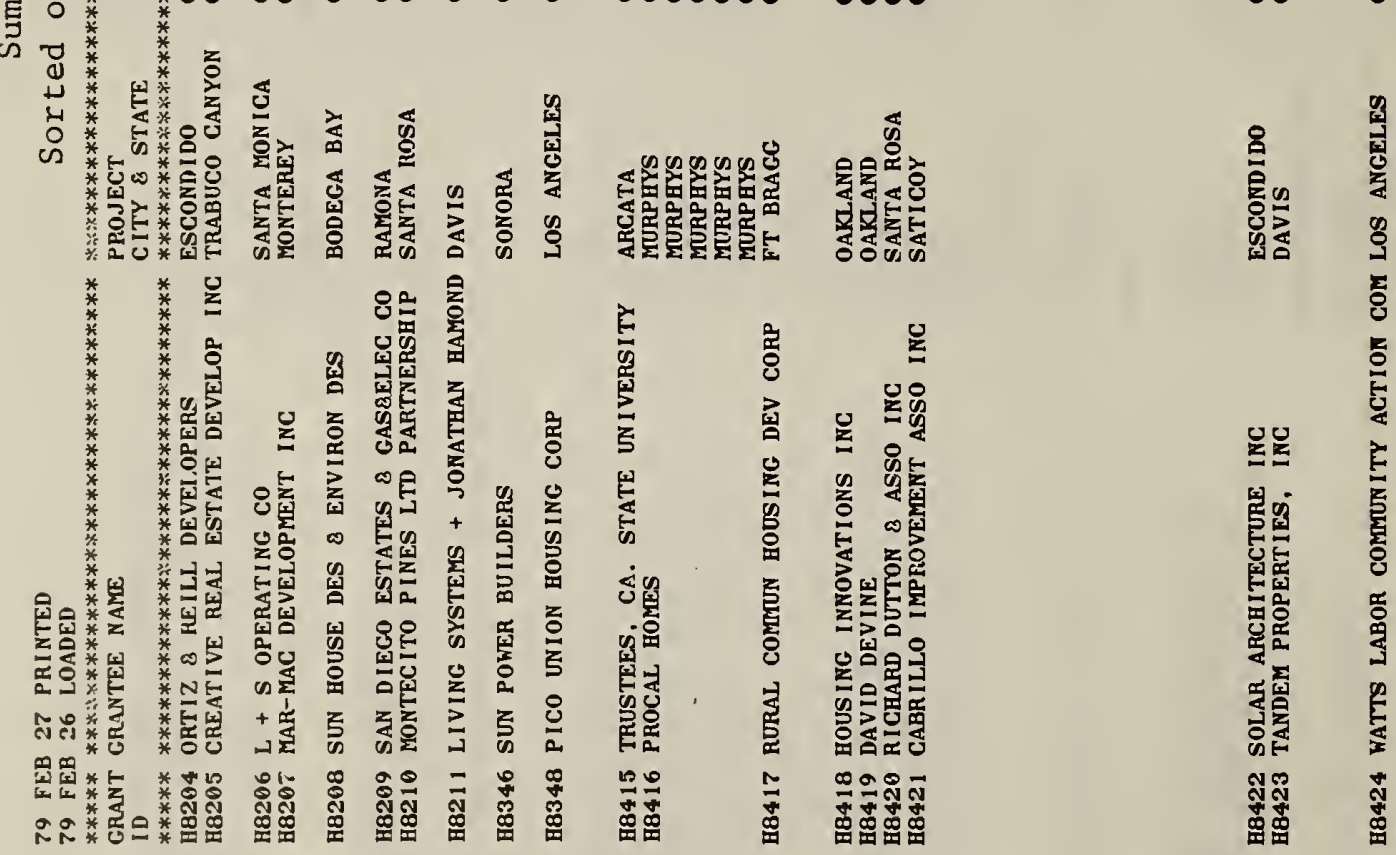




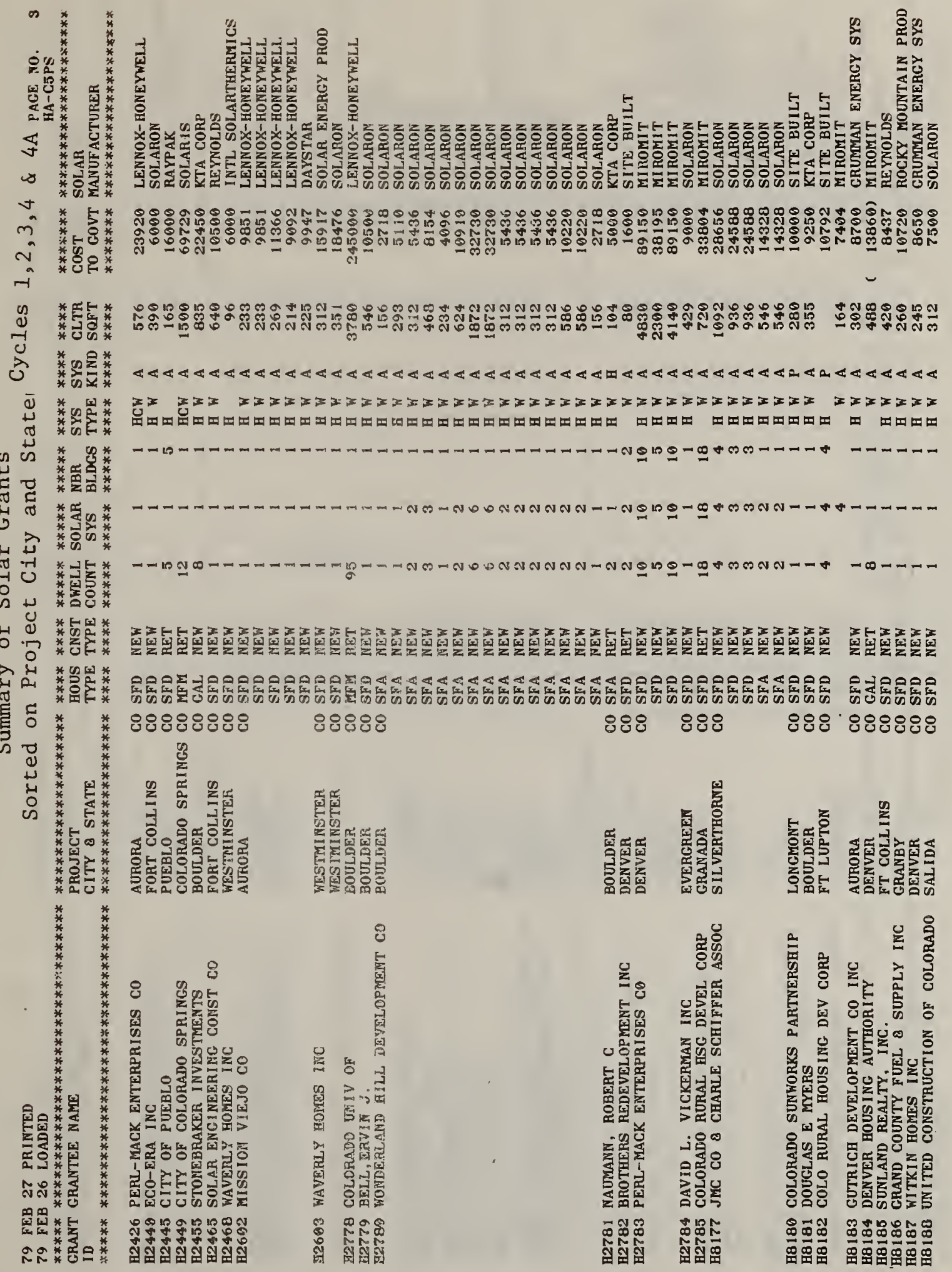




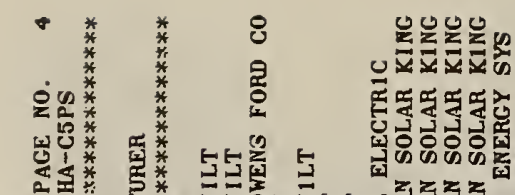

4 ;

寸

4 * * *

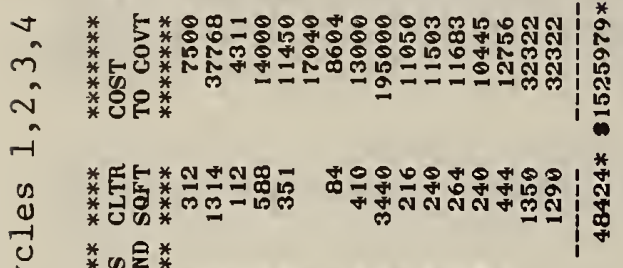

崩

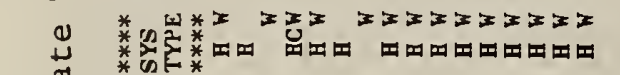

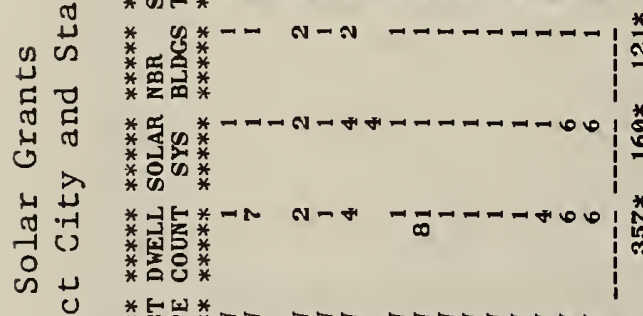

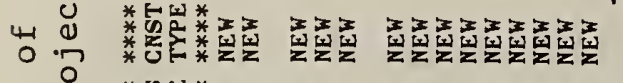

不品 * *

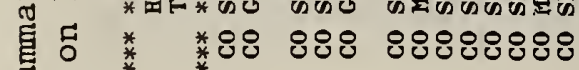

के चु

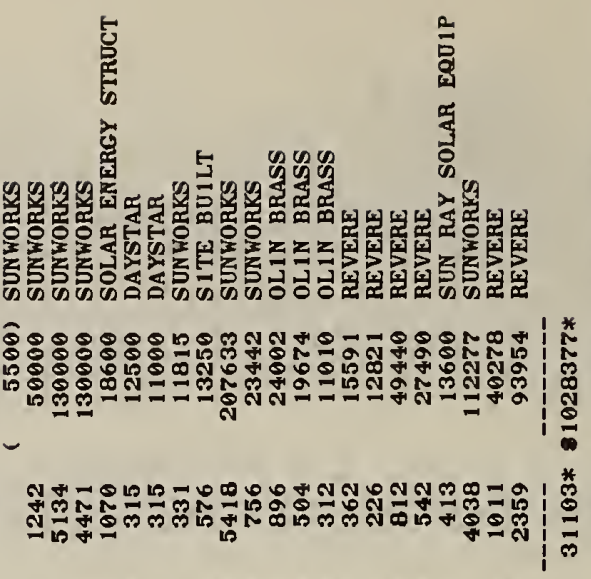

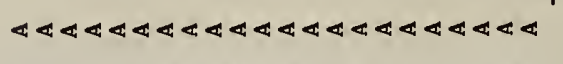

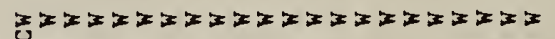

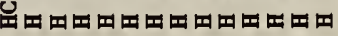

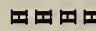

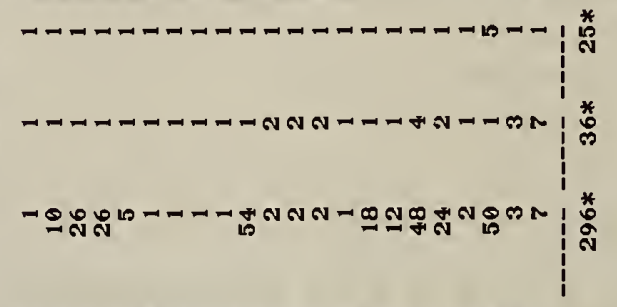

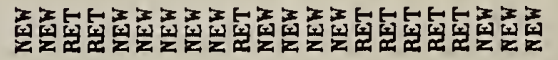

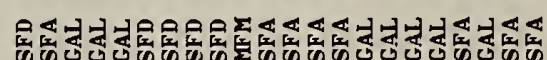

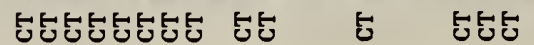

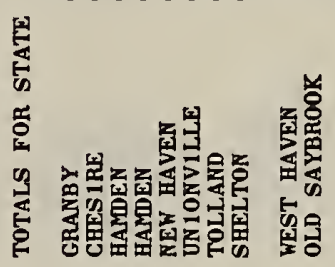

密

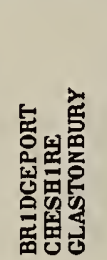

坣

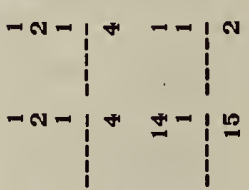

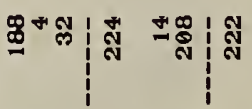

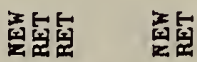

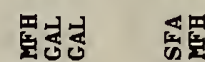

붐임 뙴욤

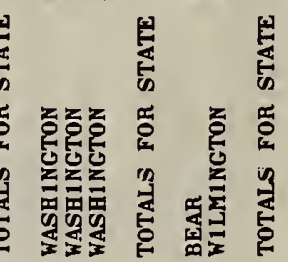

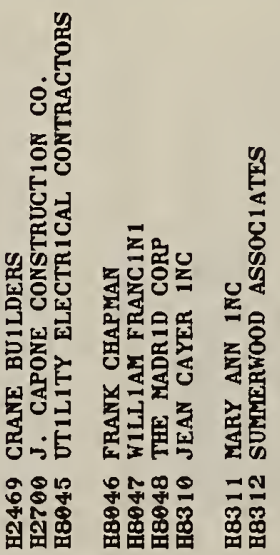

怘曾

品

思

$\frac{1}{4}$

窟

롱

密

要

ฟั้

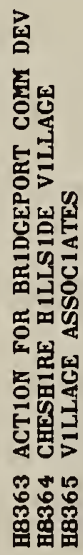

芯

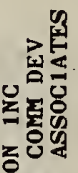




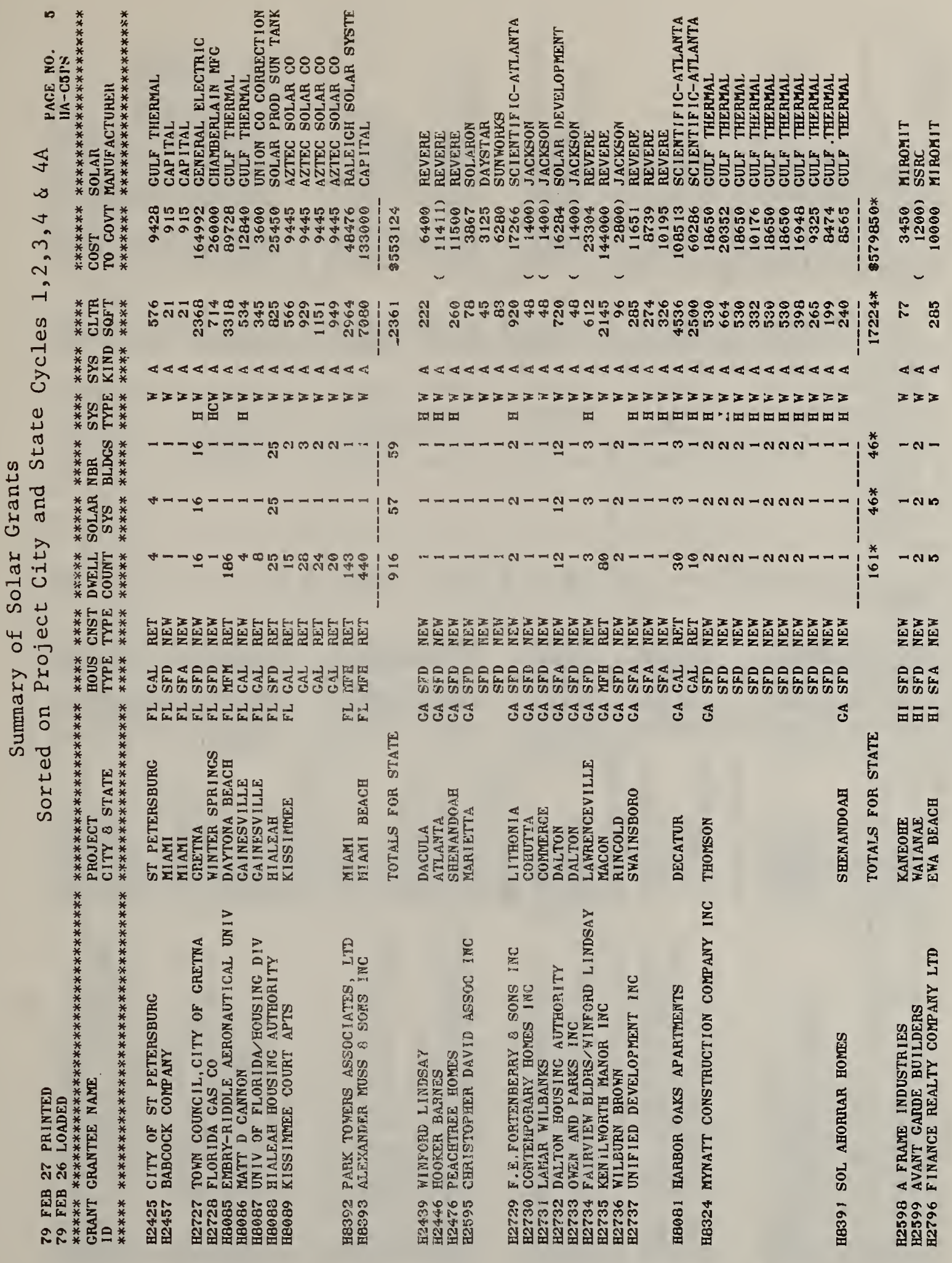




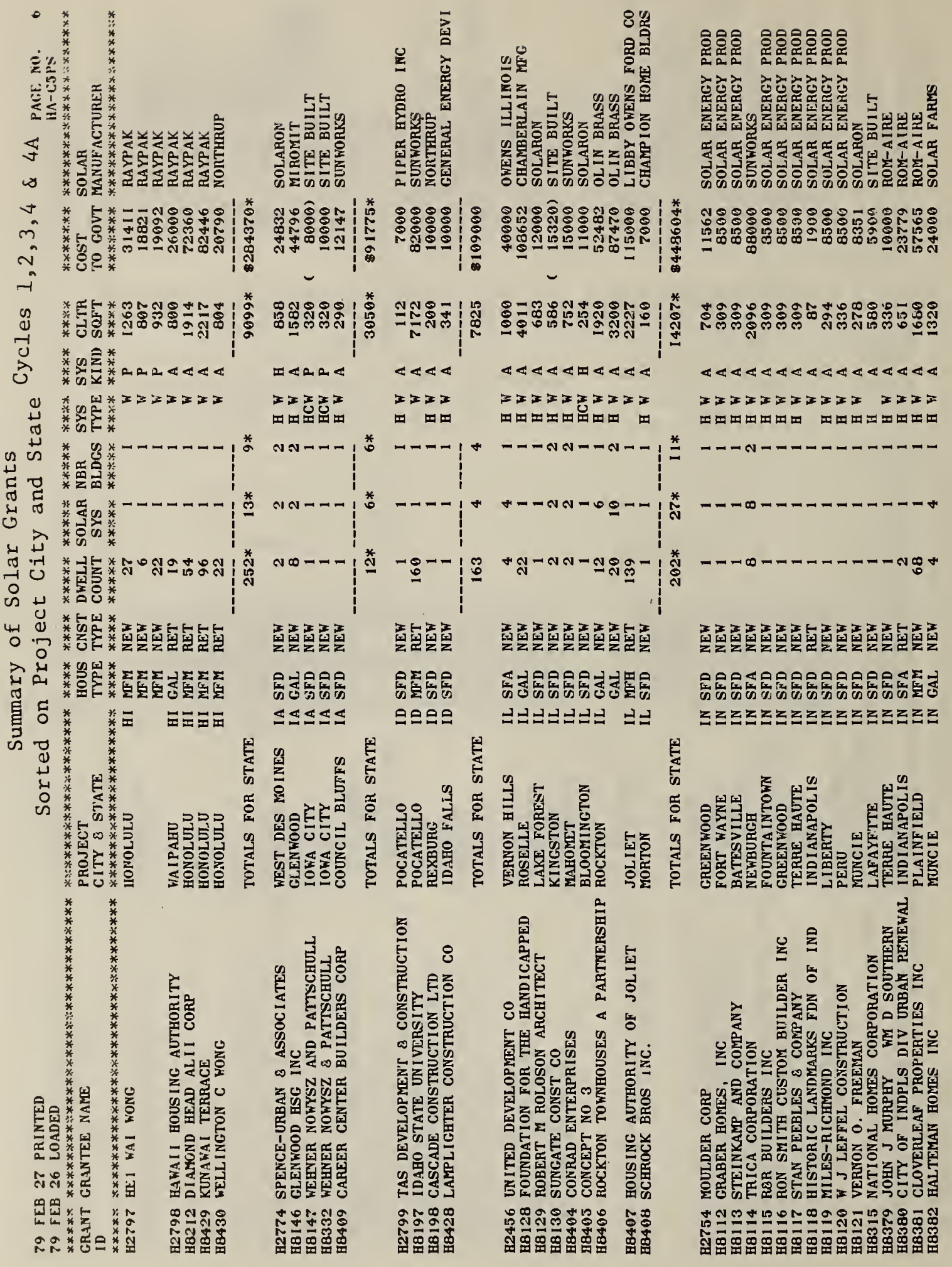




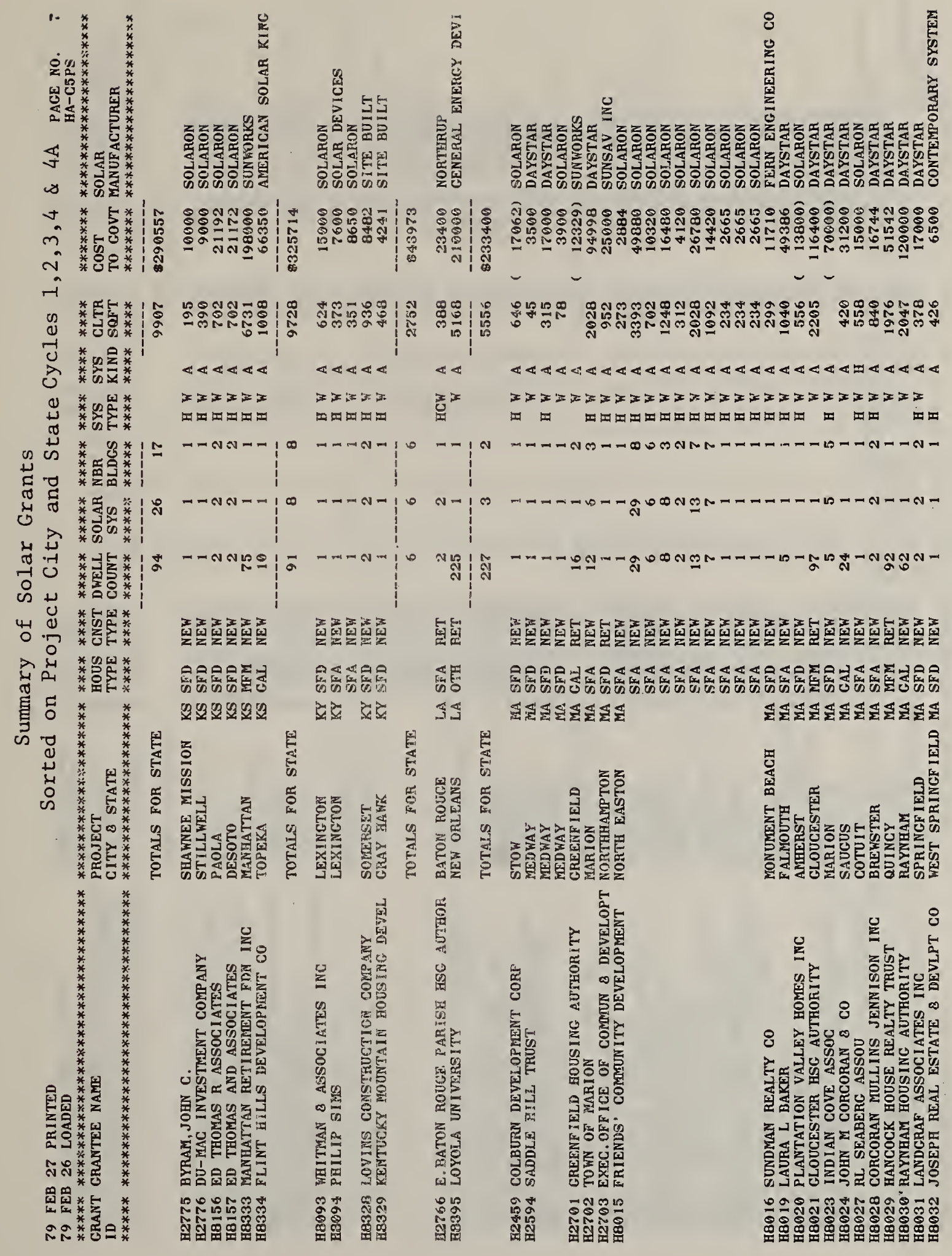




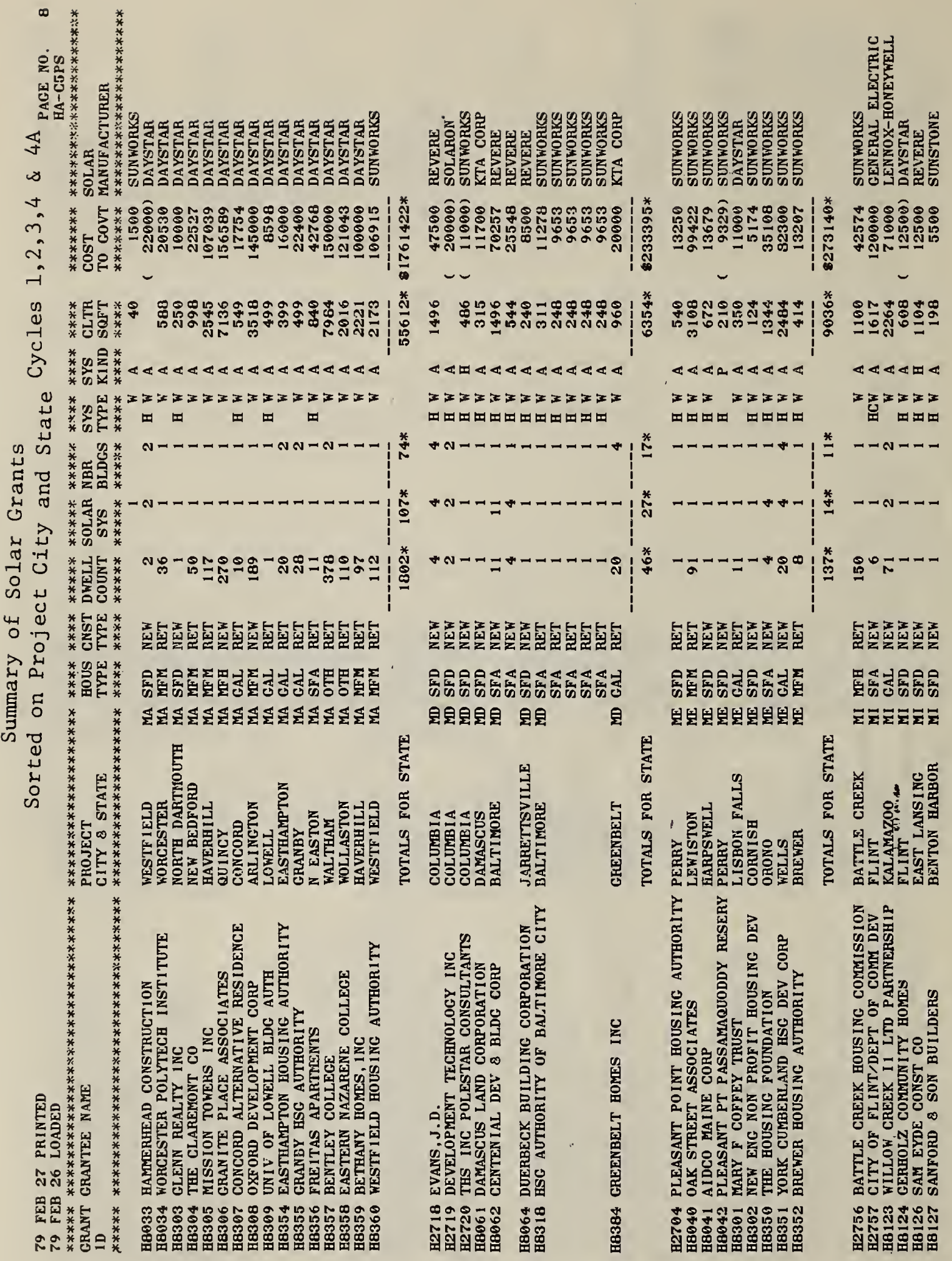




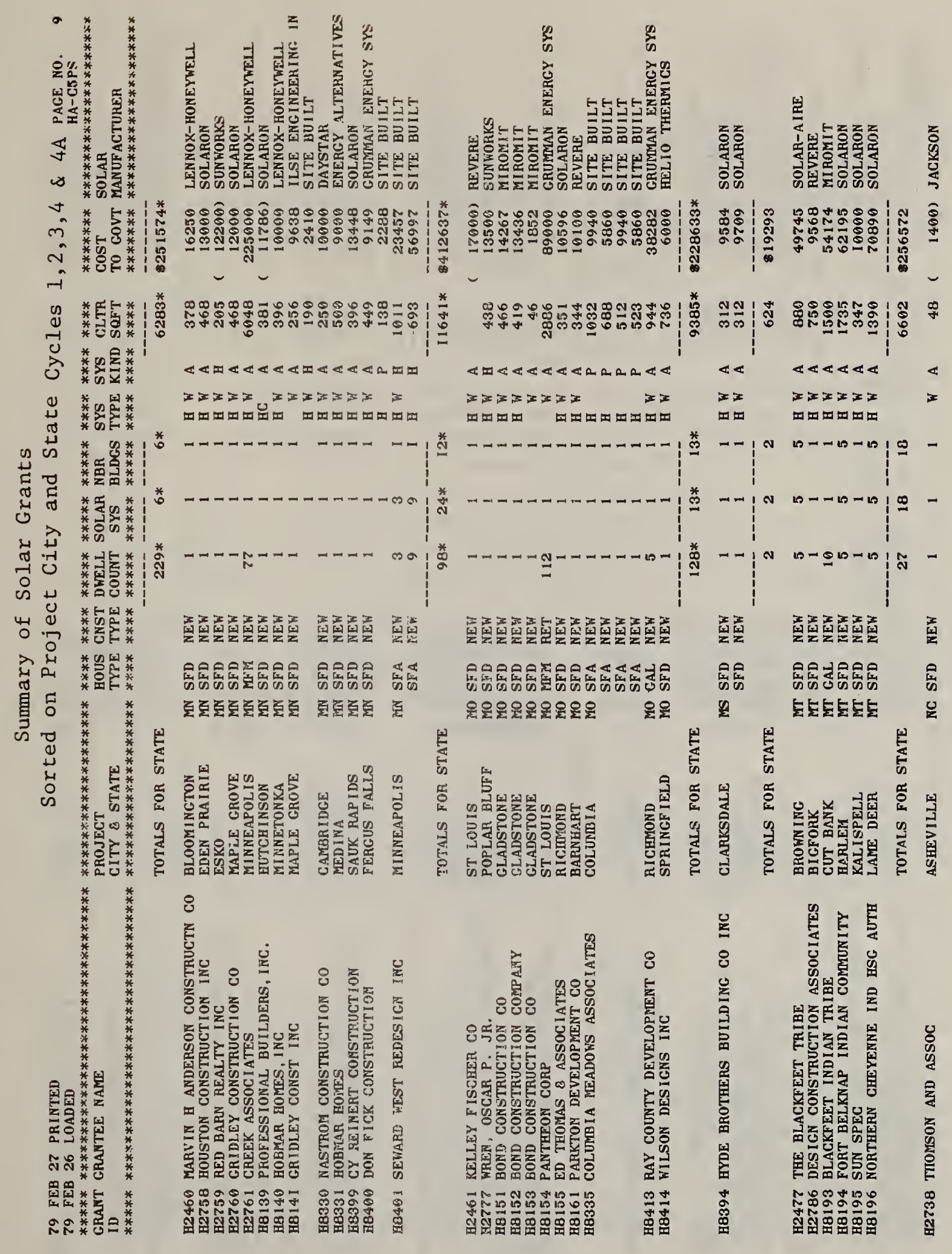




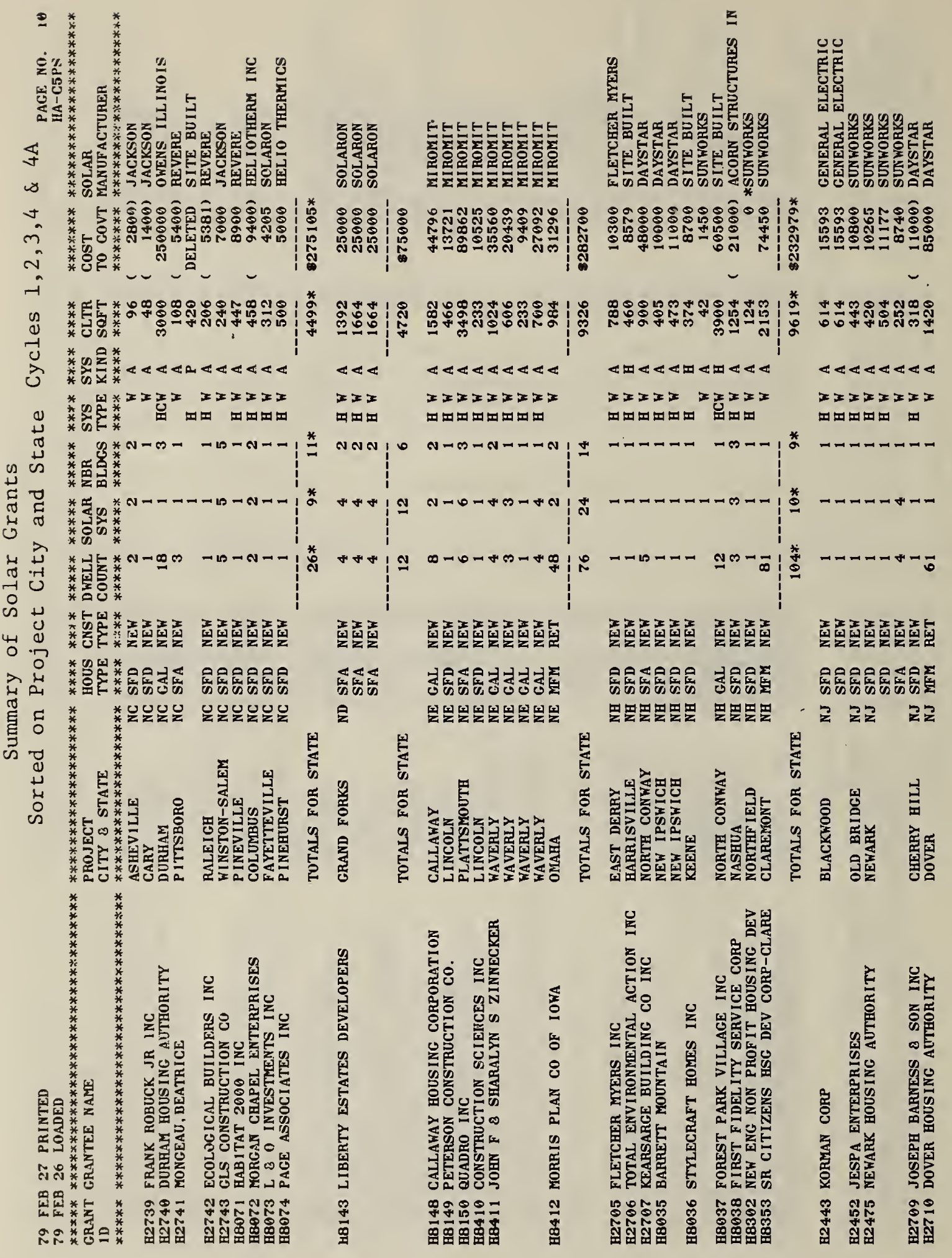




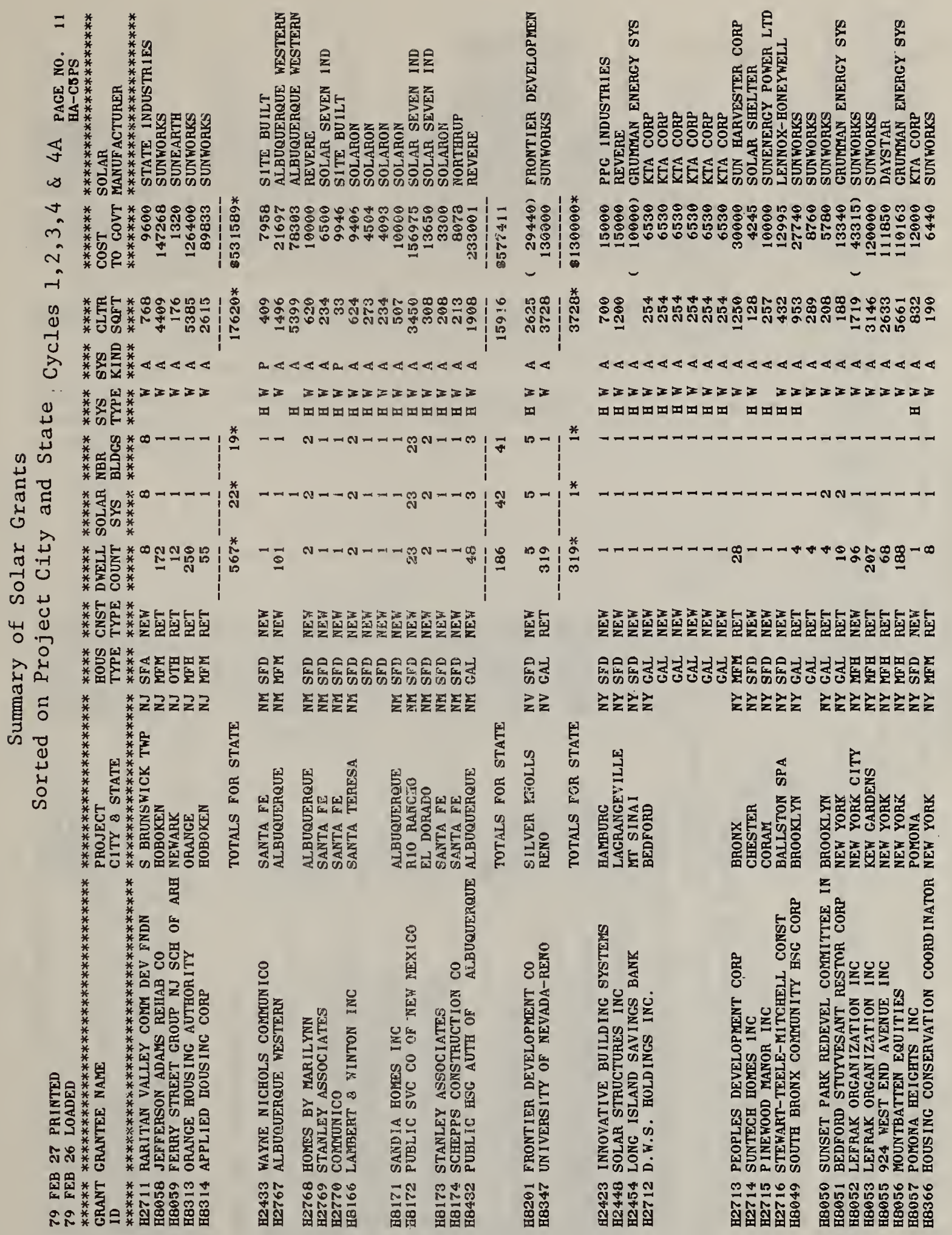




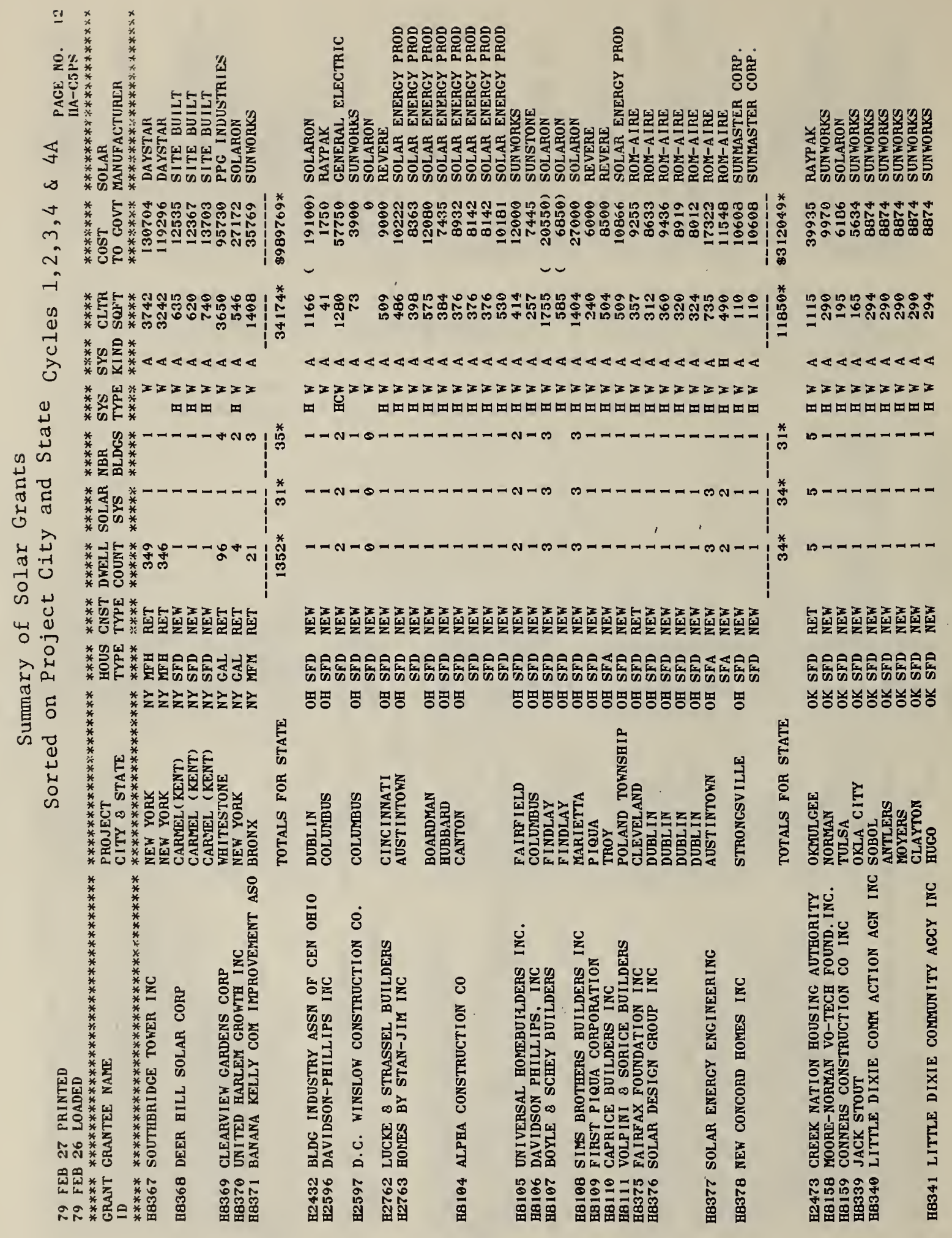




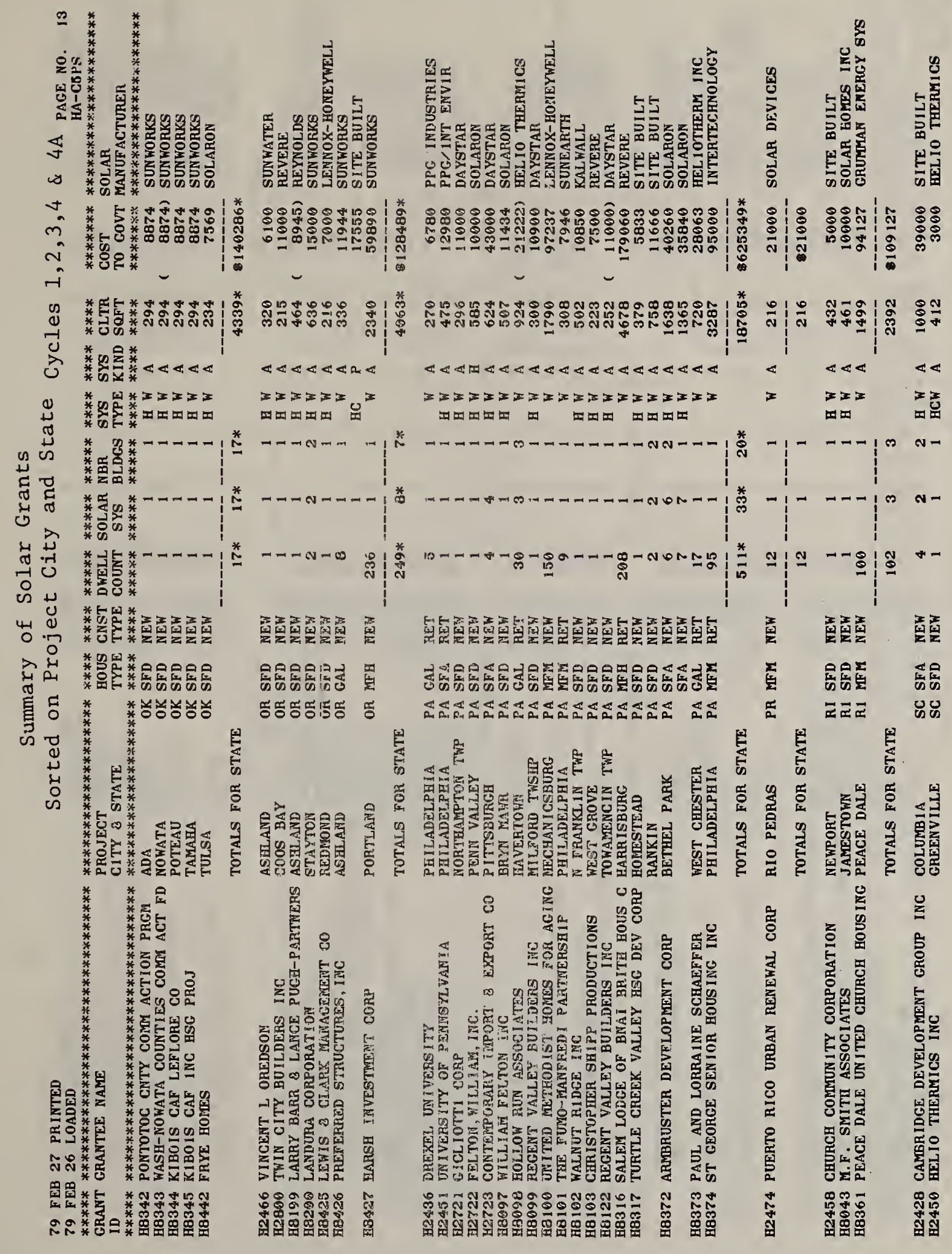




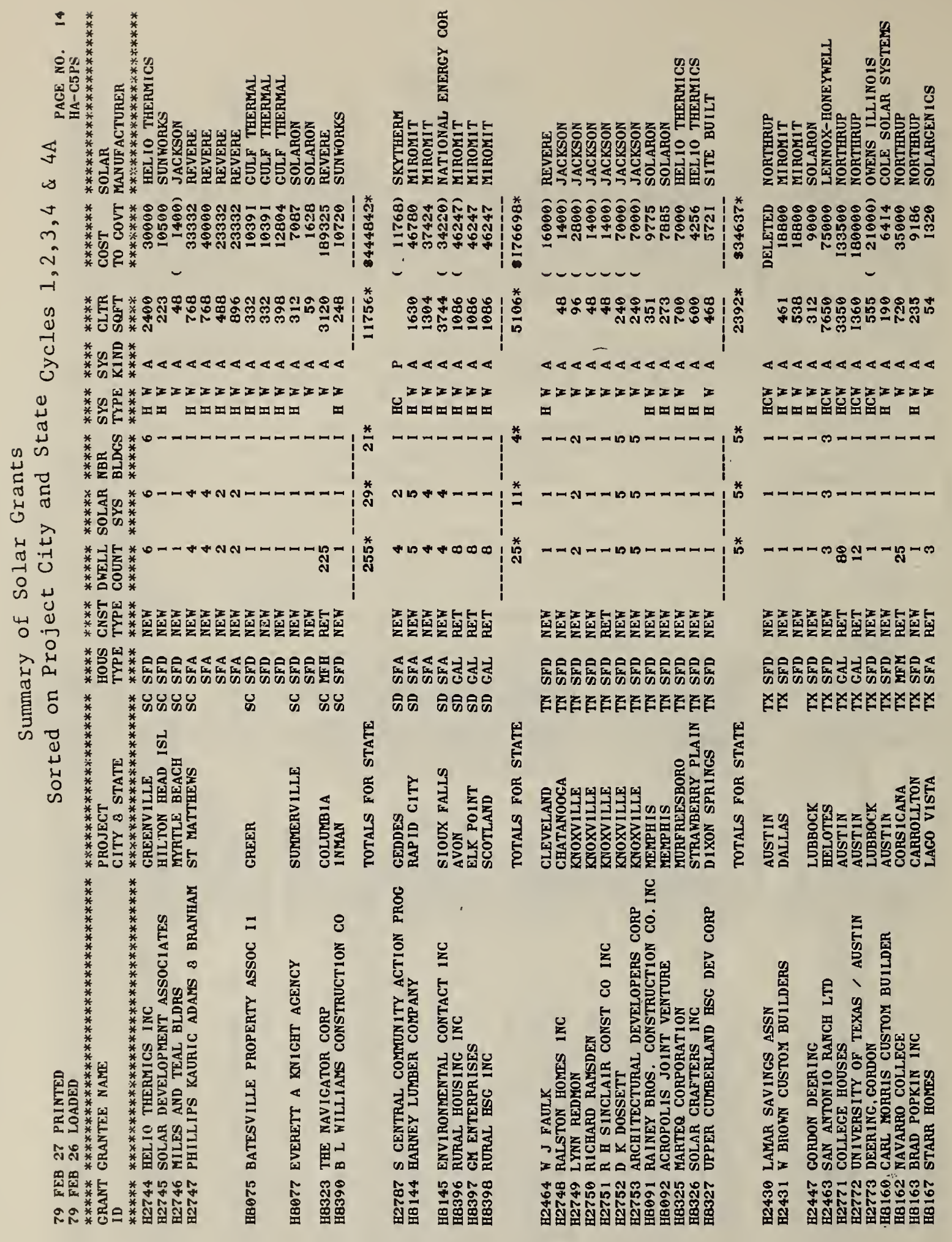




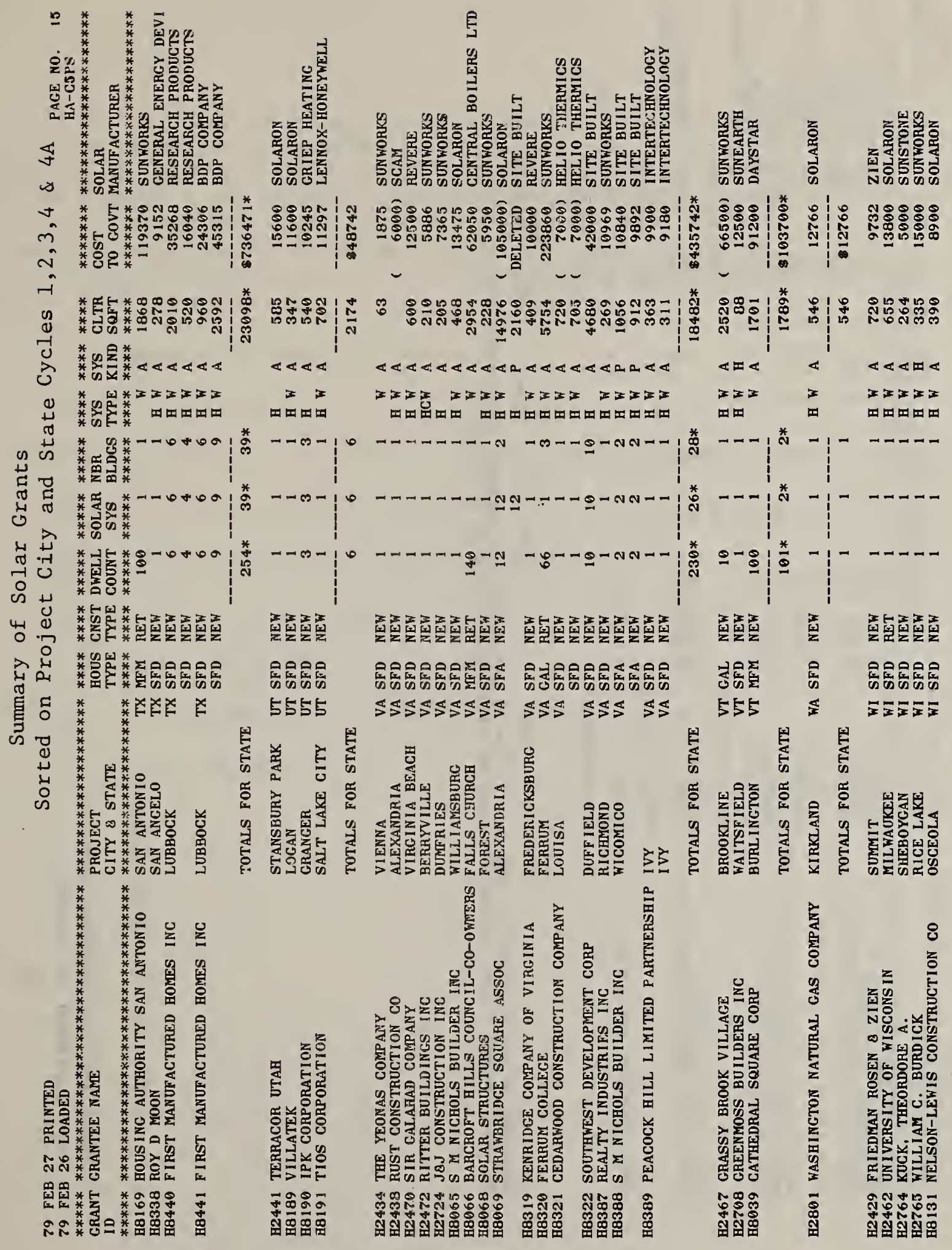



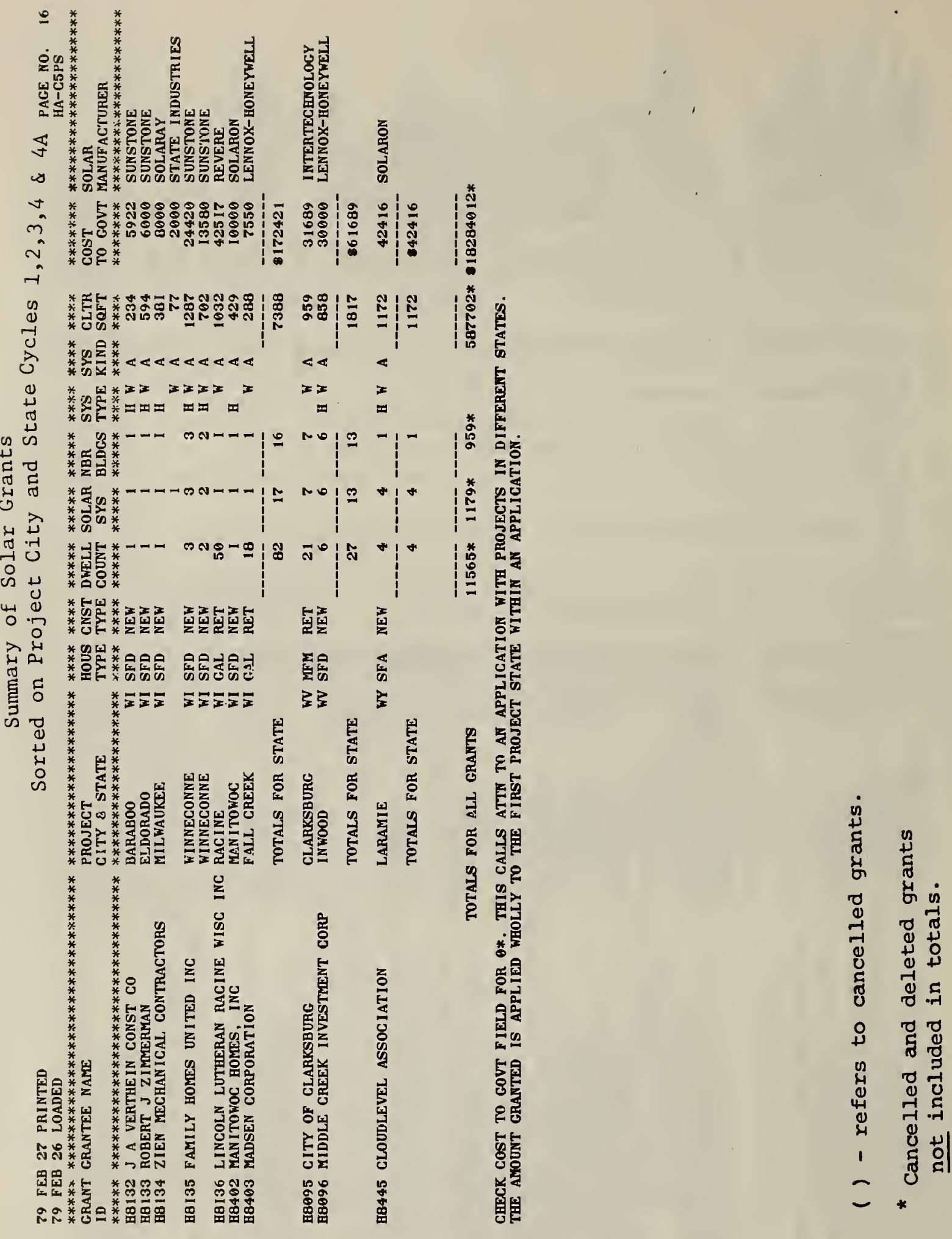


\section{GRANT FILE DATA BAR GRAPHS}

In the following bar graphs, certain data elements contained in the Grant file have been summarized and used to give a visual profile of the grants awarded by HUD to date in the Solar Demonstration Program. All of these data elements are also contained in the computer reports shown in Sections 4.1 through 4.4 . The bar graphs were produced on the NBS UNIVAC 1108 computer with FORTRAN programs developed at NBS, and then plotted by the NBS Versatec 1200A plotter.

The bar graphs included here are:

Number of Grants vs Cycles

Number of Grants vs Collector Area Distribution (Sq.Ft.)

Number of Dwelling Units vs Cycles

Number of Dwelling Units vs Cycles and New/Retrofit

Number of Dwelling Units vs Dwelling Units Distribution (States - AL to MT)

Number of Dwelling Units vs Dwelling Units Distribution (States - NC to WY) 


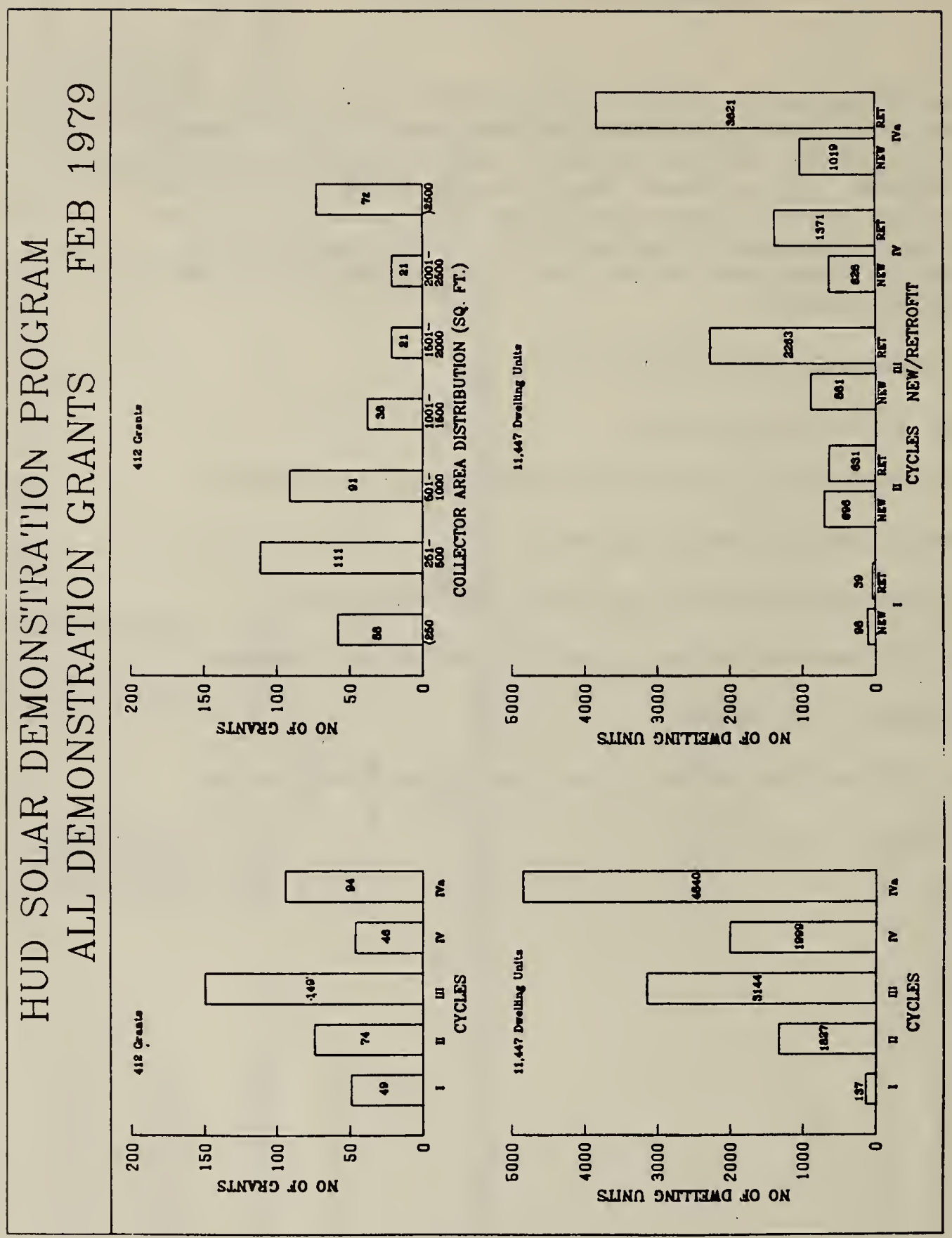




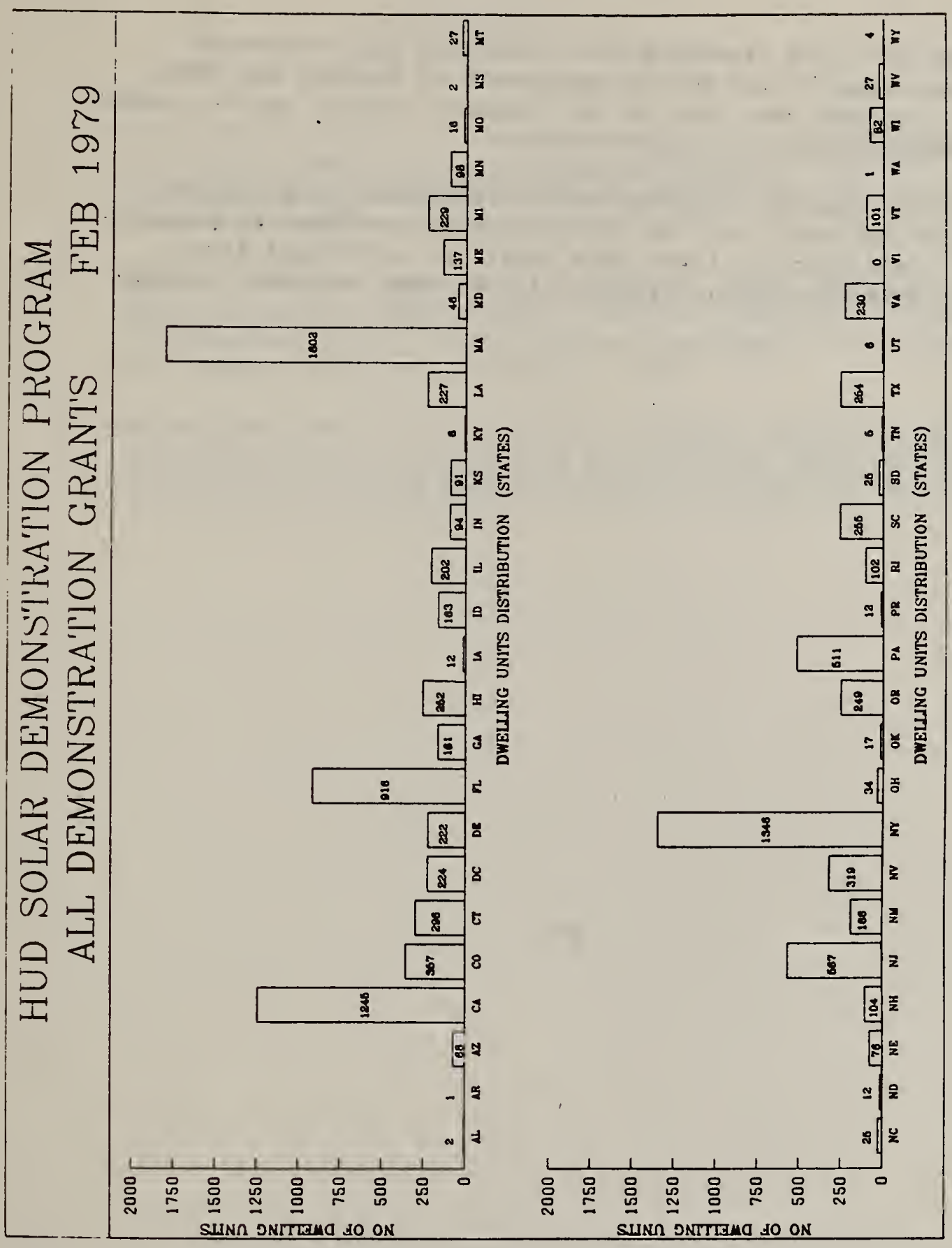




\section{SUMMARY}

The Grant file data presented here represent data collected throughout four award cycles by the Department of Housing and Urban Development. Included were some of the computer reports and bar graphs which were produced from this file of data.

The computer reports described here, with Cycle 5 and Passive Initiative data included, will be available from the Franklin Research Center (FRC) (see page 2) later this year. If additional grant cycles occur, updated computer reports will be made available through the FRC. 


\section{REFERENCES}

1. Christopher, Patricia M. and Krzewick, Joan E., Residential Solar Data Center Data Resources and Reports, National Bureau of Standards Interagency Report 79-1762 (Washington, D.C.: National Bureau of Standards, June, 1979).

2. Residential Solar Demonstration Program, Solar Energy Program, Cycle 4, H-8300, Request for Grant Application H-8300 (Washington, D. C.: United States Department of Housing and Urban Development, November, 1977).

3. Christopher, Patricia M. and Vogt, Michael, User's Guide for Online Retrieval of Solar Data Using MIRADS, Draft 1, Prepared for the United States Department of Housing and Urban Development, June, 1979. 

NBS.II4A (REV. 0.78)

\begin{tabular}{|c|c|}
\hline $\begin{array}{c}\text { U.S. OEPT. OF COMM. } \\
\text { BIBLIOGRAPHIC DATA } \\
\text { SHEET }\end{array}$ & $\begin{array}{c}\text { 1. PUBLICATION OR REPORT NO. } \\
\text { NBSIR 79-1923 }\end{array}$ \\
\hline
\end{tabular}

4. TITLE AND SUBTITLE

RESIDENTIAL SOLAR DATA CENTER GRANT REPORTS

7. AUTHOR(S)

Patricia M. Christopher and Martin J. Aronoff

9. PERFORMING ORGANIZATION NAME AND ADDRESS

NATIONAL BUREAU OF STANDARDS

DEPARTMENT OF COMMERCE

WASHINGTON, DC 20234

12. SPONSORING ORGANIZATION NAME AND COMPLETE ADDRESS (Street, City, state, zIP)

Department of Housing and Urban Development

Office of the Assistant Secretary for Policy Dexelopment and Division of Energy, Building Technology and Standards

Washington, D.C. 20410

15. SUPPLEMENTARY NOTES

[ Document describes a computer program; SF-185, FIPS Software Summary, is attached.

16. ABSTRACT (A 200-word or less factual summary of most sigrificant information. If documest includes a significant bibliography or literature survey, mention it here.)

The Residential Solar Data Center project staff in the Center for Building Technology, National Bureau of Standards, is responsible for the establishment and operation of a computerized database containing non-instrumented residential data generated by the Solar Heating and Cooling Demonstration Program sponsored by the Department of Energy and the Department of Housing and Urban Development (HUD). This document includes computer reports of data contained in the Grant file, one of six computer files comprising the database. These reports contain data recorded on applications submitted to HUD by organizations or individual builders applying for grants to build solar energy systems in new and/or existing homes. To date, approximately 450 grants have been awarded in the first four award cycles.

17. KEY WORDS (six to twelve entries; alphabetical order; capitalize only the first letter of the first key word unless a propor name; soparated by semicolons)

Automatic data processing; computer reports; grant data; residential buildings; solar database; solar energy system; solar heating and cooling.

\begin{tabular}{|c|c|c|}
\hline $\begin{array}{l}\text { 18. AVAILABILITY } \\
\text { | | For Official Distribution. Do Not Release to NTIS }\end{array}$ & $\begin{array}{l}\text { 19. SECURITY CLASS } \\
\text { (THIS REPORT) } \\
\text { UNCLASSIFIED }\end{array}$ & $\begin{array}{l}\text { 21. NO. OF } \\
\text { PRINTED PAGES } \\
73\end{array}$ \\
\hline $\begin{array}{l}\text { I I Order From Sup. of Doc. U.S. Government Printing Office, Washington, DC } \\
\text { 20402, SD Stock No. SNO03-003- }\end{array}$ & $\begin{array}{l}\text { 20. SECURITY CLASS } \\
\text { (THIS PAGE) }\end{array}$ & 22. Price \\
\hline $\begin{array}{l}\text { [x Order From National Technical Information Service (NTIS), Springfield, } \\
\text { VA. } 22161\end{array}$ & UNCLASSIFIED & $\$ 5.25$ \\
\hline
\end{tabular}




